\title{
Algorithmic Extensions of DiraC's Theorem
}

\author{
Fedor V. Fomin* \\ fomin@ii.uib.no
}

\author{
Petr A. Golovach* \\ petr.golovach@ii.uib.no \\ Kirill Simonov* \\ kirillsimonov@gmail.com
}

\author{
Danil Sagunov ${ }^{\dagger}$ \\ danilka.pro@gmail.com
}

\begin{abstract}
In 1952, Dirac proved the following theorem about long cycles in graphs with large minimum vertex degrees: Every $n$-vertex 2-connected graph $G$ with minimum vertex degree $\delta \geq 2$ contains a cycle with at least $\min \{2 \delta, n\}$ vertices. In particular, if $\delta \geq n / 2$, then $G$ is Hamiltonian. The proof of Dirac's theorem is constructive, and it yields an algorithm computing the corresponding cycle in polynomial time. The combinatorial bound of Dirac's theorem is tight in the following sense. There are 2-connected graphs that do not contain cycles of length more than $2 \delta+1$. Also, there are non-Hamiltonian graphs with all vertices but one of degree at least $n / 2$. This prompts naturally to the following algorithmic questions. For $k \geq 1$,

(A) How difficult is to decide whether a 2-connected graph contains a cycle of length at least $\min \{2 \delta+k, n\} ?$

(B) How difficult is to decide whether a graph $G$ is Hamiltonian, when at least $n-k$ vertices of $G$ are of degrees at least $n / 2-k$ ?

The first question was asked by Fomin, Golovach, Lokshtanov, Panolan, Saurabh, and Zehavi. The second question is due to Jansen, Kozma, and Nederlof. Even for a very special case of $k=1$, the existence of a polynomial-time algorithm deciding whether $G$ contains a cycle of length at least $\min \{2 \delta+1, n\}$ was open. We resolve both questions by proving the following algorithmic generalization of Dirac's theorem: If all but $k$ vertices of a 2-connected graph $G$ are of degree at least $\delta$, then deciding whether $G$ has a cycle of length at least $\min \{2 \delta+k, n\}$ can be done in time $2^{\mathcal{O}(k)} \cdot n^{\mathcal{O}(1)}$.

The proof of the algorithmic generalization of Dirac's theorem builds on new graph-theoretical results that are interesting on their own.
\end{abstract}

*Department of Informatics, University of Bergen, Norway.

${ }^{\dagger}$ St. Petersburg Department of V.A. Steklov Institute of Mathematics, Russia. 


\section{Contents}

1 Introduction 3

2 Overview of the proof 6

3 Preliminaries and classical theorems $\quad 10$

4 Generalized theorems

5 Long $(s, t)$-Cycle

6 Long Erdös-Gallai $(s, t)$-Path 21

6.1 Erdös-Gallai decompositions and structures . . . . . . . . . . . . . 21

6.2 Algorithm for Long ERdős-Gallai $(s, t)$-PATH $\ldots \ldots \ldots \ldots$. . . . . . . . . .

7 Algorithm for small vertex covers

8 Finding almost Hamiltonian cycles 45

8.1 Small separator lemma . . . . . . . . . . . . . . . . . 45

8.2 Main theorem . . . . . . . . . . . . . . . . . . . 49

9 Dirac decomposition $\quad 52$

10 Long Dirac Cycle: Putting all together $\quad 60$

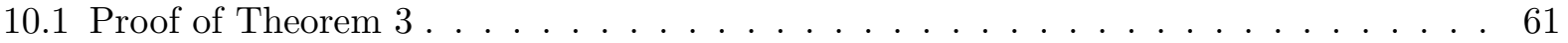

10.2 Last piece: proof of Lemma $20 \ldots \ldots \ldots$. . . . . . . . . . . . . . . . .

11 Conclusion $\quad \mathbf{7 2}$

11.1 Tightness of results . . . . . . . . . . . . . . . 72

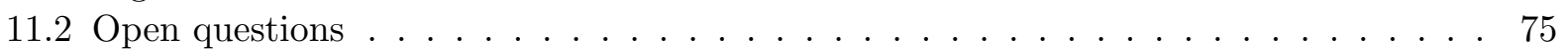




\section{Introduction}

The fundamental theorem of Dirac from 1952 guarantees the existence of a Hamiltonian cycle in a graph with a large minimum vertex degree.

Theorem 1 (Dirac [Dir52, Theorem 3]). If every vertex of an n-vertex graph $G$ is of degree at least $n / 2$, then $G$ is Hamiltonian, that is, contains a Hamiltonian cycle.

Theorem 1 follows from a more general statement of Dirac about long cycles in a graph.

Theorem 2 (Dirac Dir52, Theorem 4]). Every n-vertex 2-connected graph $G$ with minimum vertex degree $\delta(G) \geq 2$, contains a cycle with at least $\min \{2 \delta(G), n\}$ vertices.

Both Dirac's theorems were the first instances of results that developed into one of the core areas in Extremal Graph Theory. One of the main questions in this research domain is to establish vertex degree characterization of Hamiltonian graphs and conditions enforcing long paths or cycles in graphs. The (very) incomplete list of results in this area includes the classical theorems of Erdös and Gallai [EG59], Ore [Ore60], Bondy and Chvátal [BC76], Pósa [P6́2], Meyniel [Mey73, and Bollobás and Brightwell [BB93, see also the Wikipedia entry on the Hamiltonian path ${ }^{1}$ The chapters of Bondy [Bon95] and Bollobás [Bol95] in the Handbook of Combinatorics, as well as Chapter 3 in the Extremal Graph Theory book [Bol78] provide excellent introduction to this important part of graph theory. The survey of $\mathrm{Li}[\mathrm{Li13}]$ is a comprehensive (but a bit outdated) overview of the area. After almost 70 years, the field remains active, see for example the very recent proof of the Woodall's conjecture by Li and Nung [LN21].

Computing long cycles and paths is also an important topic in parameterized complexity. It served as a test-bed for developing several fundamental algorithmic techniques including the color coding of Alon, Yuster and Zwick [AYZ95, the algebraic approaches of Koutis and Williams [Kou08, Wil09], matroids-based methods [FLPS16], and the determinants-sum technique of Björklund from his FOCS 2010 Test of Time Award paper Bjö14. We refer to [FK13, [KW16], and [CFK 15a, Chapter 10] for an overview of algorithmic ideas and techniques developed for computing long paths and cycles in graphs.

Despite the tremendous progress in graph-theoretical and algorithmic studies of longest cycles, all the developed tools do not answer the following natural and "innocent" question. By Theorem 2 , deciding whether a 2-connected graph $G$ contains a cycle of length at least $\min \{2 \delta(G), n\}$ can be trivially done in polynomial time by checking degrees of all vertices in $G$.

Question 1: Is there a polynomial time algorithm to decide whether a 2-connected graph $G$ contains a cycle of length at least $\min \{2 \delta(G)+1, n\}$ ?

The methods developed in the extremal Hamiltonian graph theory do not answer this question. The combinatorial bound in Theorem 2 is known to be sharp; that is, there exist graphs that have no cycles of length at least $\min \{2 \delta(G)+1, n\}$. Since the extremal graph theory studies the existence of a cycle under certain conditions, such type of questions are beyond its applicability. The techniques of parameterized algorithms do not seem to be much of use here either. Such algorithms compute a cycle of length at least $k$ in time $2^{\mathcal{O}(k)} \cdot n^{\mathcal{O}(1)}$, which in our case is $2^{\mathcal{O}(\delta(G))} \cdot n^{\mathcal{O}(1)}$. Hence when $\delta(G)$ is, for example, at least $n^{1 / 100}$, these algorithms do not run in polynomial time.

Similarly, the existing methods do not answer the question about another "tiny algorithmic step" from Dirac's theorem, what happens when all vertices of $G$ but one are of large degree?

\footnotetext{
$\sqrt[1]{\text { https://en.wikipedia.org/wiki/Hamiltonian_path }}$
} 
Question 2: Let $v$ be a vertex of the minimum degree of a 2-connected graph $G$. Is there a polynomial time algorithm to decide whether $G$ contains a cycle of length at least $\min \{2 \delta(G-v), n\} ?$

(We denote by $G-v$ the induced subgraph of $G$ obtained by removing vertex $v$.) Note that graph $G-v$ is not necessarily 2-connected and we cannot apply Theorem 2 to it.

The incapability of existing techniques to answer Questions 1 and 2 was the primary motivation for our work. We answer both questions affirmatively and in a much more general way. Our result implies that in polynomial time one can decide whether $G$ contains a cycle of length at least $2 \delta(G-B)+k$ for $B \subseteq V(G)$ and $k \geq 0$ as long as $k+|B| \in \mathcal{O}(\log n)$. (We denote by $G-B$ the induced subgraph of $G$ obtained by removing vertices of $B$.) To state our result more precisely, we define the following problem.

LONG DiRAC CYCLE parameterized by $k+|B|$

Input: $\quad$ Graph $G$ with vertex set $B \subseteq V(G)$ and integer $k \geq 0$.

Task: Decide whether $G$ contains a cycle of length at least $\min \{2 \delta(G-$ $B),|V(G)|-|B|\}+k$.

In the definition of LONG DiRAC CyCle we use the minimum of two values for the following reason. The question whether an $n$-vertex graph $G$ contains a cycle of length at least $2 \delta(G-B)+k$ is meaningful only for $\delta(G-B) \leq n / 2$. Indeed, for $\delta(G-B)>n / 2, G$ does not contain a cycle of length at least $2 \delta(G-B)+k>n$. However, even when $\delta(G-B)>n / 2$, deciding whether $G$ is Hamiltonian, is still very intriguing. By taking the minimum of the two values, we capture both interesting situations.

The main result of the paper is the following theorem providing an algorithmic generalization of Dirac's theorem.

Theorem 3 (Main Theorem). On an n-vertex 2-connected graph $G$, LONG DiRAC CyClE is solvable in time $2^{\mathcal{O}(k+|B|)} \cdot n^{\mathcal{O}(1)}$.

In other words, LONG DIRAC CYCLE is fixed-parameter tractable parameterized by $k+|B|$ and the dependence on the parameters is single-exponential. This dependence is asymptotically optimal up to the Exponential Time Hypothesis (ETH) of Impagliazzo, Paturi, and Zane [IPZ01]. Solving LONG DiRAC CyCle in time $2^{o(k)} \cdot n^{\mathcal{O}(1)}$ even with $B=\emptyset$ yields recognizing in time $2^{o(n)}$ whether a graph is Hamiltonian. A subexponential algorithm deciding Hamiltonicity would fail ETH. In Theorem 17 we show that solving LONG DIRAC CYCLE in time $2^{o(|B|)} \cdot n^{\mathcal{O}(1)}$ even for $k=1$ would contradict ETH as well. It is also NP-complete to decide whether a 2-connected graph $G$ has a cycle of length at least $(2+\varepsilon) \delta(G)$ for any $\varepsilon>0$ (Theorem 18).

The 2-connectivity requirement in the statement of the theorem is important-without it LONG DiRAC CYCLE is already NP-complete for $k=|B|=0$. Indeed, for an $n$-vertex graph $G$ construct a graph $H$ by attaching to each vertex of $G$ a clique of size $n / 2$. Then $H$ has a cycle of length at least $2 \delta(H) \geq n$ if and only if $G$ is Hamiltonian. However, when instead of a cycle we are looking for a long path, the 2-connectivity requirement could be omitted. More precisely, consider the following problem.

Long Dirac Path parameterized by $k+|B|$

Input: $\quad$ Graph $G$ with vertex set $B \subseteq V(G)$ and integer $k \geq 0$.

Task: $\quad$ Decide whether $G$ contains a path of length at least $\min \{2 \delta(G-$ $B),|V(G)|-|B|-1\}+k$. 
Theorem 3 yields the following.

Corollary 1. On a connected $n$-vertex graph $G$, LONG DIRAC PATH is solvable in time $2^{\mathcal{O}(k+|B|)}$. $n^{\mathcal{O}(1)}$.

Indeed, when $G$ is connected, the graph $G+v$, obtained by adding a vertex $v$ and making it adjacent to all vertices of the graph, is 2-connected. The minimum vertex degree of $G+v$ is equal to $\delta(G)+1$, and $G$ has a path of length at least $t$ if and only if $G+v$ has a cycle of length at least $t+2$.

Theorem 3 answers several open questions from the literature. Fomin, Golovach, Lokshtanov, Panolan, Saurabh and Zehavi in $\left[\mathrm{FGL}^{+} 20 \mathrm{a}\right.$ asked about the parameterized complexity of problems (with parameter $k$ ) where for a given (2-connected) graph $G$ and $k \geq 1$, the task is to check whether $G$ has a path (cycle) with at least $2 \delta(G)+k$ vertices. By Theorem 3 and Corollary 1 (the case $B=\emptyset)$, both problems are fixed-parameter tractable.

Jansen, Kozma, and Nederlof in [JKN19] conjectured that if at least $n-k$ vertices of graph $G$ are of degree at least $n / 2-k$, then deciding whether $G$ contains a Hamiltonian cycle can be done in time $2^{\mathcal{O}(k)} \cdot n^{\mathcal{O}(1)}$. Theorem 3 resolves this conjecture. Indeed, if $G$ is Hamiltonian, it is 2-connected. Then let $B,|B| \leq k$, be the set of vertices such that every vertex from $V(G) \backslash B$ is of degree (in $G$ ) at least $n / 2-k$. Then $\delta(G-B) \geq n / 2-k-|B| \geq n / 2-2 k$. If $n-|B| \leq n-2 k-2|B|$, we put $k^{\prime}=|B|$, otherwise we put $k^{\prime}=n-2 \delta(G-B)$. Note that because $2 \delta(G-B) \geq n-4 k$, in both cases we have that $k^{\prime} \leq 4 k$. Also by the choice of $k^{\prime}, \min \{2 \delta(G-B), n-|B|\}+k^{\prime}=n$ and hence $G$ has a cycle of length at least $\min \{2 \delta(G-B), n-|B|\}+k^{\prime}$ if and only if $G$ is Hamiltonian. By Theorem 3 , deciding whether $G$ has a cycle of length at least $\min \{2 \delta(G-B), n-|B|\}+k^{\prime}$ can be done in time $2^{\mathcal{O}\left(k^{\prime}+|B|\right)} \cdot n^{\mathcal{O}(1)}=2^{\mathcal{O}(k)} \cdot n^{\mathcal{O}(1)}$. Interestingly, while the conjecture of Jansen, Kozma and Nederlof follows from the statement of Theorem 3 , to prove the theorem, we need to resolve this conjecture directly.

We state Theorem 3 for the decision variant of the problem. However, the proof is constructive and the corresponding cycle can be found within the same running time. Note that standard selfreduction arguments are not applicable here because deleting or contracting edges could change the minimum vertex degree.

Related work. Until very recently, graph-theoretical and algorithmic studies of the longest paths and cycles coexisted in parallel universes without almost any visible interaction. In 1992, Häggkvist [Ḧ̈2], as a corollary of his structural theorem, provided an algorithm that decides in time $n^{\mathcal{O}(k)}$ whether a graph with the minimum vertex degree at least $n / 2-k$ is Hamiltonian. In 2019, Jansen, Kozma, and Nederlof in [JKN19] gave two algorithms of running times $2^{\mathcal{O}(k)} \cdot n^{\mathcal{O}(1)}$ that decide whether the input graph $G$ is Hamiltonian when either the minimum vertex degree of $G$ is at least $n / 2-k$ or at least $n-k$ vertices of $G$ are of degree at least $n / 2$. The first result of Jansen, Kozma, and Nederlof strongly improves the algorithm of Häggkvist. However, the methods they use, like the structural theorem of Häggkvist [Ḧ̈2], are specific for Hamiltonicity and are not applicable for the more general problem of computing the longest cycle. Second, their parameterized algorithms work only in one of the scenarios: either when all vertices are of degree at least $n / 2-k$ or when at least $n-k$ vertices are of degree at least $n / 2$. Whether both scenarios could be combined, that is, the existence of a parameterized algorithm deciding Hamiltonicity when $n-k$ vertices are of degree at least $n / 2-k$, was left open.

Fomin, Golovach, Lokshtanov, Panolan, Saurabh and Zehavi in $\mathrm{FGL}^{+}$20a] gave an algorithm that in time $2^{\mathcal{O}(k)} \cdot n^{\mathcal{O}(1)}$ decides whether a 2-connected graph $G$ contains a cycle of length at least $d+k$, where $d$ is the degeneracy of $G$. Since the minimum vertex degree $\delta(G)$ does not exceed the degeneracy of $G$, this result also implies an algorithm for finding a cycle of length at least $\delta(G)+k$ in 2-connected graphs. 
None of the works [JKN19] and [FGL ${ }^{+}$20a] could be used to address Questions 1 and 2, the very special cases of Theorem 3 .

More generally, Theorem 3 fits into a popular trend in parameterized complexity called "above guarantee" parameterization. The general idea of this paradigm is that the natural parameterization of, say, a maximization problem by the solution size is not satisfactory if there is a lower bound for the solution size that is sufficiently large. For example, there always exists a satisfying assignment that satisfies half of the clauses or there is always a max-cut containing at least half the edges. Thus nontrivial solutions occur only for the values of the parameter that are above the lower bound. This indicates that for such cases, it is more natural to parameterize the problem by the difference of the solution size and the bound. Since the work of Mahajan and Raman [MR99] on MAx SAT and MAX Cut, the above guarantee approach was successfully applied to various problems, see e.g. $\mathrm{AGK}^{+} 10, \mathrm{CJM}^{+} 13$, GP16a, GKLM11, GvIMY12, GP16b, GRSY07, LNR ${ }^{+14}$, MRS09]. In particular, [BCDF19] and [ $\left.\mathrm{FGL}^{+} 20 \mathrm{~b}\right]$ study the longest path above the shortest $s, t$-path and the girth of a graph.

\section{Overview of the proof}

The original proof of Dirac is not constructive because it does not provide any procedure for constructing a cycle of length at least $2 \delta(G)$. There are algorithmic proofs of Dirac's theorem; see, e.g., the thesis of Locke [Loc83]. The idea of Locke's proof that also provides a polynomial-time algorithm for constructing a cycle of length at least $2 \delta(G)$ is to start from some cycle and to grow by inserting new vertices and short paths. Thanks to the conditions on the graph's degrees, such a procedure always constructs a cycle of the required length. On a very general level, our proof of Theorem 3 uses the same strategy. For an instance $(G, B, k)$ of LONG DiRAC CYClE, we try to grow a cycle iteratively. However, enlarging the cycle by "elementary" improvements could get stuck with a cycle of length significantly smaller than $\min \{2 \delta(G-B),|V(G)|-|B|\}+k$. It appears that the cycles that cannot be improved by "elementary" operations induce a very particular structure in a graph. These structural theorems play a crucial role in our algorithm.

The main technical contribution is the new graph decomposition that we call Dirac decomposition. The formal definition is given in Section 9. Dirac decomposition is defined for a cycle $C$ in $G$. Let $C$ be a cycle of length less than $2 \delta(G-B)+k$. Informally, the components of Dirac decomposition are connected components in $G-V(C)$. (For an intuitive description of the decomposition, we will assume that $B=\emptyset$. Handling vertices of $B$ requires more technicalities - we have to refine the graph and work with its refinement.) Since $G$ is 2-connected, we can reach $C$ by a path starting in such a component in $G$. One of the essential properties of Dirac decomposition is a limited number of vertices in $V(C)$ that have neighbors outside of $C$. In fact, we can choose two short paths $P_{1}$ and $P_{2}$ in $C$ (and short means that their total length is of order $k$ ) such that all connections between connected components of $G-V(C)$ and $C$ go through $V\left(P_{1}\right) \cup V\left(P_{2}\right)$. The second important property is that each connected component of $G-\left(V\left(P_{1}\right) \cup V\left(P_{2}\right)\right)$ is connected with $P_{i}$ in $G$ in a very restricted way: The maximum matching size between its vertex set and the vertex set of $P_{i}$ is at most one.

Dirac decomposition appears to be very useful for algorithmic purposes. For a cycle $C$ and a vertex set $B$, given a Dirac decomposition for $C$ and $B$, in time $2^{\mathcal{O}(k+|B|)} \cdot n^{\mathcal{O}(1)}$ we either solve the problem or succeed in enlarging $C$ (Theorem 16). We also provide an algorithm that either constructs a Dirac decomposition in polynomial time or obtains additional structural information that again can be used either to solve the problem or to enlarge the cycle. More precisely, first, we need to eliminate the "extremal" cases. When $\delta(G-B) \in \mathcal{O}(k)$, the classical result of Alon, Yuster, 
and Zwick [AYZ95] solves the problem in time $2^{\mathcal{O}(k+|B|)} \cdot n^{\mathcal{O}(1)}$. Another extremal case is when $|B| \leq k$ and $\delta(G-B) \geq \frac{n}{2}-k$. In that case, for solving LONG DiraC CyCle, we have to decide in time $2^{\mathcal{O}(k)} \cdot n^{\mathcal{O}(1)}$ whether $G$ is almost Hamiltonian, i.e., a cycle in $G$ that cover all but $\mathcal{O}(k)$ vertices. The existence of such an algorithm for Hamiltonian cycles was conjectured in [JKN19] and Theorem 7 settles this conjecture. We give an overview of the proof of Theorem 7 later in this section. If we are in none of the extremal cases, then (Lemma 20) in polynomial time we can either (a) enlarge the cycle $C$, or (b) compute a vertex cover of $G-B$ of size at most $\delta(G-B)+2 k$, or

(c) compute a Dirac decomposition. In cases (a) and (c), we can proceed iteratively. For the case (b) we give an algorithm that solves the problem in time $2^{\mathcal{O}(k+|B|)} \cdot n^{\mathcal{O}(1)}$ (Theorem 6).

The most critical and challenging component of the proof is Theorem 16 about algorithmic properties of Dirac decomposition. We use the properties of Dirac decomposition to show that an enlargement of a cycle $C$ of length at most $2 \delta(G-B)+k-1$ can be done in a very particular way. By an extension of Dirac's existential theorem, Theorem 14, we can assume that $C$ is of length at least $2 \delta(G-B)$. The most interesting and not-trivial situation that could occur is that for some vertices $x \in V\left(P_{1}\right)$ and $y \in V\left(P_{2}\right)$, we replace the shortest $(x, y)$-path in $C$ by a detour with a particular property. This detour leaves $x$, moves to a vertex $s$ of some 2-connected component of $G-V(C)$, visits some vertices in this component, leaves it from a vertex $t$, and goes to vertex $y$. Since the length of the longest $(x, y)$-path in $C$ is at least $\delta(G-B)$, to decide whether such a detour exists, it is sufficient to solve the following problem. For vertices $s, t$ of a 2-connected graph $G$, decide whether $G$ contains an $(s, t)$-path of length at least $\delta(G-B)+k$. We give an algorithm solving this problem in time $2^{\mathcal{O}(k+|B|)} \cdot n^{\mathcal{O}(1)}$ (Theorem 5). The combinatorial bound that an $(s, t)$-path of length $\delta(G)$ always exists if $G$ is 2-connected, is the classical theorem of Erdös and Gallai [EG59, Theorem 1.16]. Because of that, we name the problem of computing an $(s, t)$-path of length at least $\delta(G-B)+k$ by the Long ERdős-Gallai $(s, t)$-PATH problem. Long ERdős-Gallai $(s, t)$-PATH is an interesting problem on its own, and to prove Theorem 5, we use another structural result which we call ErdösGallai decomposition. Similar to Dirac decomposition, this decomposition is very useful from the algorithmic perspective. In Section 6, we define this decomposition, provide efficient algorithms for constructing it, and use it to solve Long ERDős-Gallai $(s, t)$-PATH. Another interesting component of the solution to LONG ERDös-GALLAI $(s, t)$-PATH is the algorithm for computing the longest cycle passing through two specified vertices (Theorem 4). We are not aware of the previous work in parameterized algorithms on this natural problem.

Figure 1 displays the most important steps of the proof and the dependencies between them. In the remaining part of this section, we highlight the ideas behind each of the auxiliary steps (Theorems 4, 5, 6, and 7) in the proofs of Theorem 3 and Theorem 16 .

The first auxiliary problem whose solution we use in the proof of Theorem 5 is the following.

LONG $(s, t)$-CYCLE parameterized by $k$

Input: $\quad$ Graph $G$ with two vertices $s, t \in V(G)$ and integer $k \geq 0$.

Task: Decide whether there is a cycle in $G$ of length at least $k$ that passes through $s$ and $t$.

When $s \neq t$, an equivalent formulation is to decide whether $G$ contains two internally disjoint $(s, t)$-paths of total length at least $k$. In Section 5 we prove the following theorem.

Theorem 4. LONG $(s, t)$-CYCLE is solvable in time $2^{\mathcal{O}(k)} \cdot n^{\mathcal{O}(1)}$.

While the first idea to design an algorithm claimed in Theorem 4 would be to use the color coding technique of Alon, Yuster and Zwick [AYZ95], this idea does not work directly. The reason 


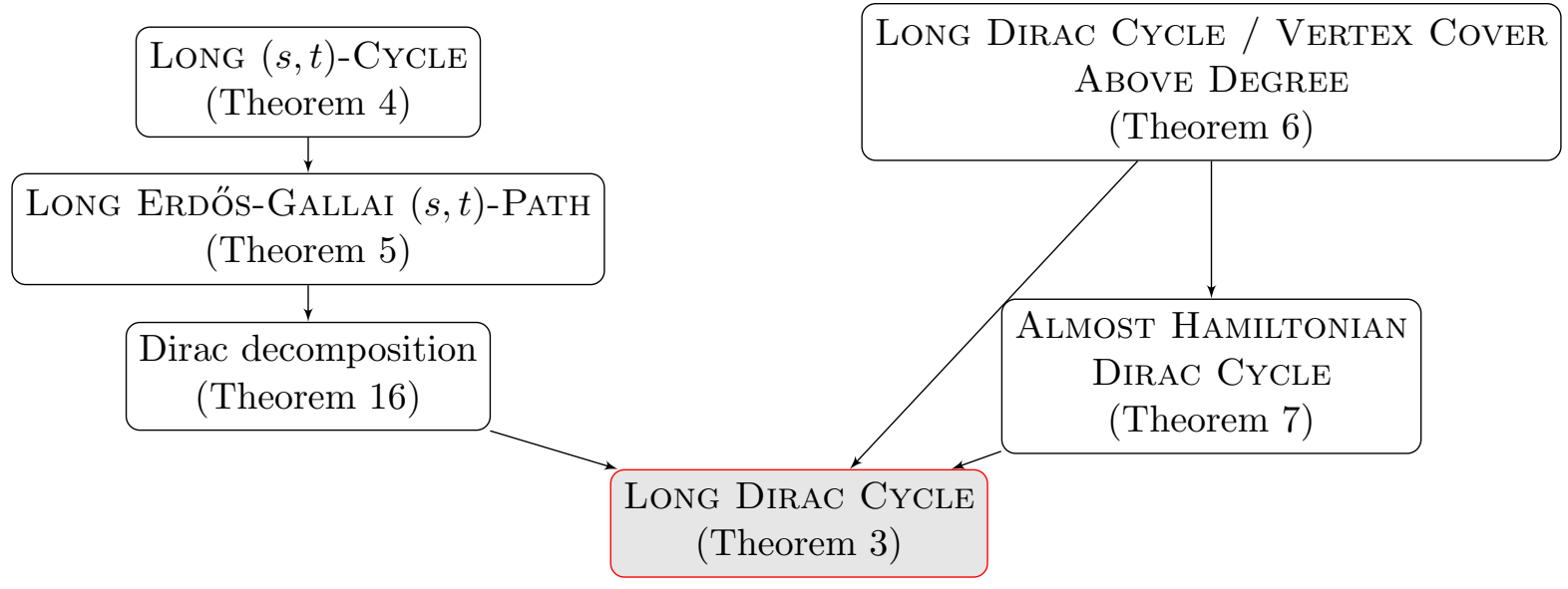

Figure 1: The main steps and connections in the proof of Theorem 3.

is that color coding can be used only to find in the claimed running time the cycle whose length is of order of $k$. However, it is quite possible that the lengths of all solutions are much larger than $k$; in such situation color coding cannot be applied directly. Our approach in proving Theorem 4 builds on ideas from $\left[\mathrm{FLP}^{+} 18\right.$, Zeh16, where a parameterized algorithms for finding a directed $(s, t)$-path and a directed cycle of length at least $k$ were developed. The main idea of the proof is the following. First, we use color coding to verify whether the considered instance has a solution composed by two $(s, t)$-paths of total length at most $3 k$. If the instance has a solution, we return it and stop. Otherwise, we conclude that the total length of the paths of every solution is at least $3 k+1$. This allows to use structural properties of paths. Let $P_{1}$ and $P_{2}$ be the $(s, t)$-paths of a solution of minimum total length. Then there are vertices $x_{1}$ and $x_{2}$ on $P_{1}$ and $P_{2}$, respectively, such that (i) the total length of the $\left(s, x_{1}\right)$-subpath $P_{1}^{\prime}$ of $P_{1}$ and the $\left(s, x_{2}\right)$-subpath $P_{2}^{\prime}$ of $P_{2}$ is exactly $k$, (ii) either $x_{1}=s$ or the length of the $\left(x_{1}, t\right)$-subpath $P_{1}^{\prime \prime}$ of $P_{1}$ is at least $k$, and, symmetrically, (iii) either $x_{2}=s$ or the length of the $\left(x_{2}, t\right)$-subpath $P_{2}^{\prime \prime}$ of $P_{2}$ is at least $k$. Then $P_{1}^{\prime \prime}$ and $P_{2}^{\prime \prime}$ are internally disjoint paths that are shortest disjoint paths avoiding $V\left(P_{1}^{\prime}\right) \cup V\left(P_{2}^{\prime}\right) \backslash\left\{x_{1}, x_{2}\right\}$. We use the method of random separation to distinguish the following three sets: $V\left(P_{1}^{\prime}\right) \cup V\left(P_{2}\right) \backslash\left\{x_{1}, x_{2}\right\}$, the last $\min \left\{k,\left|V\left(P_{1}\right)\right|-2\right\}$ internal vertices of $P_{1}^{\prime \prime}$, and the last $\min \left\{k,\left|V\left(P_{2}\right)\right|-2\right\}$ internal vertices of $P_{2}^{\prime \prime}$. This allows to highlight the crucial parts of the shortest solution and then find a solution.

The second problem whose solution we use in the proof of Theorem 3, comes from another classical theorem due to Erdős and Gallai from [EG59, Theorem 1.16], see also [Loc85]. For every pair of vertices $s, t$ of a 2-connected graph $G$, there is a path of length at least $\min _{v \in V(G) \backslash\{s, t\}} \operatorname{deg} v$. The proof of this result is constructive, and it implies a polynomial time algorithm that finds such a path. We define Long ERdős-Gallai $(s, t)$-PATH as follows.

Long Erdős-Gallai $(s, t)$-PATH parameterized by $k+|B|$

Input: $\quad$ Graph $G$ with vertex set $B \subseteq V(G)$, two vertices $s, t \in V(G)$ and integer $k \geq 0$.

Task: $\quad$ Decide whether $G$ contains an $(s, t)$-path of length at least $\delta(G-B)+k$.

In Section 6, we prove the following theorem. This theorem plays an important role in the proof of Theorem 16.

Theorem 5. Long ERdős-Gallai $(s, t)$-PATH is solvable in time $2^{\mathcal{O}(k+|B|)} \cdot n^{\mathcal{O}(1)}$ on 2-connected graphs. 
Similar to Long Dirac PATH, the requirement that the input graph is 2-connected is important. It is easy to prove that Long ERDös-Gallai $(s, t)$-PATH is NP-complete for $k=|B|=0$ when the input graph is not 2-connected.

To prove Theorem 5, we apply the following strategy. We take an $(s, t)$-path $P$ and try to extend it as much as possible. The principal tool in enlarging the path $P$ is Corollary 3 , which is an extension of the theorem of Erdös and Gallai that takes into account the vertices of $B$. In the extremal case, when we cannot extend the path anymore, we obtain a graph decomposition whose properties become useful from the algorithmic perspective. We call this decomposition by the name of Erdős-Gallai decomposition and prove that, in that case, the graph can be decomposed in a very particular way. Roughly speaking, after a certain refinement of the graph, the $(s, t)$-path $P$ consists of a prefix $P_{1}$ and a suffix $P_{2}$ with the following properties. These parts of the path are sufficiently far from each other in $P$. Moreover, all components of the graph $G-V\left(P_{1} \cup P_{2}\right)$, we call them Erdős-Gallai component, are connected to $P_{1}$ and $P_{2}$ in a very restricted way. Such a graph-theoretical insight helps us to characterize how a long $(s, t)$-path traverses through an Erdös-Gallai component. This property allows us to design the recursive algorithm that proves the theorem.

The next auxiliary result required for proof of Theorem 3, concerns LONG DiRAC CyCLE parameterized by the vertex cover of a graph. It is well-known, see e.g., $\mathrm{CFK}^{+} 15 \mathrm{~b}$, that a longest path in a graph $G$ could be found in time $2^{\mathcal{O}(t)} n^{\mathcal{O}(1)}$, where $t$ is the size of the minimum vertex cover of $G$. However, we need a much more refined result for the proof of the main theorem, where the parameter is not just the size of the vertex cover, but the difference between that size and $\delta(G-B)$. We define the following parameterized problem.

Long Dirac Cycle / Vertex Cover Above Degree parameterized by $p+|B|$

Input: $\quad$ Graph $G$ with vertex set $B \subseteq V(G)$, vertex cover $S$ of $G$ of size $\delta(G-B)+p$ and integer $k \geq 0$.

Task: $\quad$ Decide whether $G$ contains a cycle of length at least $2 \delta(G-B)+k$.

Section 7 is devoted to the proof of the following theorem, which we need for both Theorem 7 and Theorem 3 ,

Theorem 6. Long Dirac Cycle / Vertex Cover Above Degree is solvable in $2^{\mathcal{O}(p+|B|)}$. $n^{\mathcal{O}(1)}$ running time.

To prove Theorem 6, we establish the new structural result, Lemma 13. The lemma reduces the crucial case of the problem about the long cycle to a particular path cover problem. This equivalence becomes very handy because we can use color-coding to compute the particular path cover, and thus by the lemma, to compute a long path. In spirit, Lemma 13 is close to the classical theorem of Nash-Williams [NW71], stating that a 2-connected graph $G$ with $\delta(G) \geq(n+2) / 3$ is either Hamiltonian or contains an independent set of size $\delta(G)+1$. An extension of this theorem is due to Häggkvist [Ḧ̈2], which was used by Jansen, Kozma and Nederlof [JKN19] in their algorithm for Hamiltonicity below Dirac's condition. In our case, we cannot use the structural theorem of Häggkvist as a black box, and build on the new graph-theoretic lemma instead.

The last ingredient we need to prove Theorem 3 , is its special case when the minimum degree of $\delta(G-B)$ is nearly $\frac{n}{2}$. Specifically, the problem is defined as follows. 
Almost Hamiltonian Dirac Cycle parameterized by $k$

Input: $\quad$ Graph $G$, integer $k \geq 0$ and vertex set $B \subset V(G)$, such that $|B| \leq k$ and $\delta(G-B) \geq \frac{n}{2}-k$.

Task: $\quad$ Find the longest cycle in $G$.

Observe that for a 2-connected graph, Theorem 14 always gives a cycle of length $2 \delta(G-B) \geq n-2 k$. Thus it is more natural to state the problem in the form above, as the length of the longest cycle is necessarily between $n-2 k$ and $n$, which is at most $2 \delta(G-B)+2 k$. In other words, we look for an almost Hamiltonian cycle, in a sense that it does not cover only $\mathcal{O}(k)$ vertices. Now we state our result for Almost Hamiltonian Cycle that we prove in Section 8 .

Theorem 7. Let $G$ be a given 2-connected graph on $n$ vertices and let $k$ be a given integer. Let $B \subseteq V(G)$ be such that $|B| \leq k$ and $\delta(G-B) \geq \frac{n}{2}-k$. There is a $2^{\mathcal{O}(k)} \cdot n^{\mathcal{O}(1)}$ running time algorithm that finds the longest cycle in $G$.

The key obstacle for proving the theorem is the low-degree set $B$, since for empty $B$, we could simply apply the Nash-Williams theorem [NW71] and obtain either a Hamiltonian cycle or an independent set of size $\delta(G)+1$, and in the latter case use our result for LONG Dirac CyCLE / Vertex Cover Above Degree. Assume there exists a Hamiltonian cycle in $G$ (for almost Hamiltonian cycles the algorithm is similar), it induces a certain path cover of the vertices of $B$, where the endpoints of paths belong to $V(G) \backslash B$, and their total length is $\mathcal{O}(k)$. Such a path cover can be found by color-coding and dynamic programming in time $2^{\mathcal{O}(k)} n^{\mathcal{O}(1)}$. Now either the rest of the graph is not 2-connected, and we have a $\mathcal{O}(k)$-sized separator, or we can apply the Nash-Williams theorem and obtain a cycle covering everything except the path cover, or a large independent set. The latter case is dealt with by Theorem 6 , and for the case of the small separator we design a special algorithm that leverages the fact that the resulting components are very dense. So the main case is when the graph splits into a long cycle and the path cover. Now we crucially use that the paths in the path cover start and end outside of $B$, thus the endpoints of a path have high degree, each of them sees roughly half of the vertices of the long cycle. This makes it "hard" to not be able to insert the path somewhere in the cycle and make it longer. However, this last intuitive idea is achieved by a very intricate case analysis that constitutes the most of technical difficulty of the proof. Also, in some of the cases, we cannot make the cycle longer nor conclude that it is impossible, but instead we are able to find either a small separator or a large independent set. Again, we settle these cases by using the respective specialized algorithms.

\section{Preliminaries and classical theorems}

Graph notation. Most of the graph notation that we use here are standard and are compatible with the notation used in the textbook of Diestel [Die17]. Graphs in this paper are finite and undirected. The vertex set of a graph $G$ is denoted by $V(G)$ and the edge set of $G$ is denoted by $E(G)$. We use shorthands $n=|V(G)|$ and $m=|E(G)|$. An edge of an undirected graph with endpoints $u$ and $v$ is denoted by $u v$.

Graph $H$ is a subgraph of graph $G$ if $V(H) \subseteq V(G)$ and $E(H) \subseteq E(G)$. For a subset $S \subseteq V(G)$, the subgraph of $G$ induced by $S$ is denoted by $G[S]$; its vertex set is $S$ and its edge set consists of all the edges of $E(G)$ that have both endpoints in $S$. For $B \subseteq V(G)$, we use $G-B$ to denote the graph $G[V \backslash B]$, and for $F \subseteq E(G)$ by $G-F$ we denote the graph $(V(G), E(G) \backslash F)$. We also write $G-v$ instead of $G-\{v\}$. 
For graph $G$ and edge $u v \in E(G)$, by contracting edge $u v$ we mean the following operation. We remove $u$ and $v$ from the graph, introduce a new vertex $w_{u v}$, and connect it to all the vertices $u$ or $v$ were adjacent to. The neighborhood of a vertex $v$ in $G$ is $N_{G}(v)=\{u \in V \mid u v \in E(G)\}$ and the closed neighborhood of $v$ is $N_{G}[v]=N_{G}(v) \cup\{v\}$. For a vertex set $S \subseteq V$, we define $N_{G}[S]=\bigcup_{v \in S} N[v]$ and $N_{G}(S)=N_{G}[S] \backslash S$. We denote by $\operatorname{deg}_{G}(v)$ the degree of a vertex $v$ in graph $G$, which is just the number of edges incident with $v$. We may omit indices if the graph under consideration is clear from the context. We use $\delta(G)$ for minimum vertex degree of graph $G$.

A path $P$ in a graph is a nonempty sequence of vertices $v_{0}, \ldots, v_{k}$ such that for every $i=$ $0, \ldots, k-1$ we have $v_{i} v_{i+1} \in E(G)$ and $v_{i} \neq v_{j}$ for all $i \neq j$. Vertices $v_{0}$ and $v_{k}$ are the endpoints of path $P$ and $v_{1}, \ldots, v_{k-1}$ are internal. If $P=v_{0} v_{1} \ldots v_{k}$ is a path, then the graph obtained from $P$ by adding edge $x_{k} x_{0}$ is a cycle. The length of a path or cycle is equal to the cardinality of its edge set. The distance between vertices $u$ and $v$ in a graph $G$ is the shortest length of a path between $u$ and $v$. For vertices $s, t \in V(G)$, an $(s, t)$-path is a path with the first vertex $s$ and the last vertex $t$. A Hamiltonian path (cycle) in a graph $G$ is a path (cycle) passing through all the vertices of $G$. Two paths $P$ and $Q$ are internally disjoint if every internal vertex of one path is not a vertex of the other path, that is, $P$ and $Q$ may only share their endpoints. The concatenation of internally vertex-disjoint paths $P=v_{0}, \ldots, v_{k}$ and $Q=v_{k}, v_{k+1}, \ldots, v_{\ell}$ is $P Q=v_{0}, \ldots, v_{k}, v_{k+1}, \ldots, v_{\ell}$. Note that $P Q$ is a path if $v_{0} \neq v_{\ell}$ and is a cycle if $v_{0}=v_{\ell}$. An arc in a cycle $C$ is a path whose all edges belong to $C$. A chord of a cycle $C$ is a path connecting two non-adjacent vertices of $C$ that is internally vertex-disjoint with $C$.

An undirected graph $G$ is connected if for every pair $u, v$ of its vertices there is a path between $u$ and $v$. A vertex set $X \subseteq V(G)$ is connected if the subgraph $G[X]$ is connected. A connected component of $G$ is the subgraph induced by a maximal connected vertex subset of $G$. A connected graph $G$ with at least three vertices is 2-connected if for every $v \in V(G), G-v$ is connected. Similarly, a vertex set $X \subseteq V(G)$ is 2-connected if the subgraph $G[X]$ is 2-connected. A block of $G$ is the subgraph induced by a maximal 2-connected subset. A vertex $v$ is a cut-vertex if it belongs to at least two blocks. All other vertices of a block are inner vertices. Blocks in a graph form a forest structure (viewing each block as a vertex of the forest and two blocks are adjacent if they share a cut-vertex). The blocks corresponding to the leaves of the block-forest, are referred as leaf-blocks. A connected component is separable if it contains a cut-vertex, or equivalently, if it is not 2-connected.

A vertex cover $X$ of a graph $G$ is a subset of the vertex set $V(G)$ such that $X$ covers the edge set $E(G)$, i.e., every edge of $G$ has at least one endpoint in $X$. An independent set $I$ in a graph $G$ is a subset of the vertex set $V(G)$ such that the vertices of $I$ are pairwise nonadjacent. A path cover of a graph $G$ is a family of disjoint paths in $G$ such that every vertex of $G$ belongs to some of these paths.

Classical results. Besides Dirac's theorem from [Dir52, already stated as Theorem 1, we use the result that guarantees a long path between two fixed vertices of a 2-connected graph. Its different versions can be found throughout the works of Locke [Loc83, Loc85]. The version below is from the paper of Egawa, Glas, and Locke [EGL91, Lemma 5].

Lemma 1 (Egawa, Glas, and Locke [EGL91]). Let $G$ be a 2-connected graph with at least 4 vertices, and let $s, t \in V(G)$ be a pair of vertices in $G$, and let $d$ be an integer. If all vertices in $G$, except $s, t$ and one other vertex, have degree at least $d$, then there exists an $(s, t)$-path of length at least $d$ in $G$.

In addition, we rely on several other classical theorems. In some parts of the proof we use one more result from the Dirac's work [Dir52]. 
Theorem 8 (Dirac [Dir52]). Every graph $G$ contains a cycle of of length at least $\delta(G)+1$.

We remark that Lemma 1 and Theorem 8 are constructive in the following sense. Their proofs can be turned into polynomial time algorithms producing an $(s, t)$-path of length at least $d$ and a pa cycle of of length at least $\delta(G)+1$.

In 1976, Bondy and Chvátal [BC76] proved the following generalization of Theorem 1 that we use in Section 7. Let $G$ be an $n$-vertex graph. The closure $\mathrm{cl}(G)$ of $G$ is the graph obtained from $G$ by iteratively making two distinct vertices $u$ and $v$ adjacent whenever the sum of their degrees is at least $n$. Note that if $\operatorname{deg}_{G}(u)+\operatorname{deg}_{G}(v) \geq n$ for all pairs of vertices $u$ and $v$, then $\operatorname{cl}(G)=K_{n}$. In particular, the closure of a $n$-vertex graph satisfying the conditions of Theorem 1 is $K_{n}$.

Theorem 9 (Bondy and Chvátal [BC76]). A graph $G$ has a Hamiltonian cycle if and only if $\mathrm{cl}(G)$ has a Hamiltonian cycle.

We remark that the proof of Theorem 9 yields a polynomial time algorithm constructing from a cycle with an added edge a cycle in a graph without the new edge. Thus by repeating this argument for every added edge, we obtain a polynomial time algorithm for constructing a Hamiltonian cycle in $G$ from a Hamiltonian cycle in $\mathrm{cl}(G)$.

Another classical result that we require is the well-known Menger's theorem.

Theorem 10 (Menger's theorem, Men27, Gör00]). Let $G$ be a graph and $A, B \subseteq V(G)$ be two subsets of its vertices. Let $s$ be the minimum number of vertices separating $A$ and $B$ in $G$. There are $s$ vertex-disjoint paths going from $A$ to $B$.

Throughout the paper we are mostly working with 2-connected graphs, so we just need the following corollary of the Menger's theorem.

Corollary 2. Let $G$ be a 2-connected graphs and $A, B \subseteq V(G)$ be two subsets of its vertices such that $|A|,|B| \geq 2$. There exist two vertex-disjoint paths going from $A$ to $B$ in $G$.

Finally, we will make use of a strengthening of Dirac's theorem due to Nash-Williams [NW71]. We state it in the form following Jansen, Kozma and Nederlof [JKN19], where the following algorithmic statement is proven.

Theorem 11 (Nash-Williams [NW71, JKN19]). Let $G$ be a 2-connected graph with $n$ vertices, with $\delta(G) \geq(n+2) / 3$. Then, we can find in $G$, in time $\mathcal{O}\left(n^{3}\right)$, either a Hamiltonian cycle, or an independent set of size $\delta(G)+1$.

Parameterized algorithms. We will use several results from parameterized complexity as black boxes. Let us recall that Longest CyCle (Longest PATH) are the problems where for given graph $G$ and integer $k$, the task is to decide whether $G$ has a cycle (a path) of length at least $k$. In LONG $(s, t)$-PATH, for $s, t \in V(G)$, the task is to decide whether an $(s, t)$-path of length at leas $k$ exists. The first algorithms for LONGest CYCLE and LONGEST PATH of running time $2^{\mathcal{O}(k)} \cdot n^{\mathcal{O}(1)}$ are due to Alon, Yuster and Zwick [AYZ95. The fastest known randomized algorithm for LONGEST PATH on undirected graph is due to Björklund, Husfeldt, Kaski and Koivisto [BHKK10] and runs in time $1.657^{k} \cdot n^{\mathcal{O}(1)}$. Tsur gave the fastest known deterministic algorithm for the problem running in time $2.554^{k} \cdot n^{\mathcal{O}(1)}$ Tsu19. For LONGEST CYCLE, the current fastest randomized algorithm running in time $4^{k} \cdot n^{\mathcal{O}(1)}$ is due to Zehavi [Zeh16] and the best deterministic algorithm runs in time $4.884^{k}$. $n^{\mathcal{O}(1)}\left[\mathrm{FLP}^{+} 18\right]$. For LONG $(s, t)$-PATH the best known running time is $4.884^{k} \cdot n^{\mathcal{O}(1)}\left[\mathrm{FLP}^{+} 18\right.$.

Theorem 12 ([AYZ95, $\left.\left[\mathrm{FLP}^{+} 18\right]\right)$. Longest Path, Longest CyCle, and Long $(s, t)-$ Path admit algorithms with running time $2^{\mathcal{O}(k)} \cdot n^{\mathcal{O}(1)}$. 
We also use the following algorithms of Jansen, Kozma, and Nederlof [JKN19].

Theorem 13 ([JKN19]). If a graph $G$ has at least $n-k$ vertices of degree at least $\frac{n}{2}$ or if a graph $G$ has $\delta(G) \geq \frac{n}{2}-k$, a Hamiltonian cycle in $G$ can be found in time $2^{\mathcal{O}(k)} \cdot n^{\mathcal{O}(1)}$.

\section{Generalized theorems}

The classical theorems of Dirac and Erdös-Gallai provide bounds on the length of cycles and paths in terms of vertex degrees in graph $G$. In our algorithmic extension of Dirac's theorem, we deal with a more general problem when the cycle's length is bounded by vertex degrees of graph $G-B$. In our algorithm we use generalizations of these classical results stated for vertex degrees in graph $G-B$. These generalizations are simple and most likely they are known as a folklore. However we could not find them in the literature and prove them here for completeness.

The first theorem is the generalization of Dirac's theorem (Theorem 2): Theorem 2 is its special case with $B=\emptyset$.

Theorem 14. Let $G$ be a 2-connected n-vertex graph. For any $B \subseteq V(G)$ there exists a simple cycle in $G$ of length at least $\min \{n-|B|, 2 \delta(G-B)\}$. Moreover, there is a polynomial time algorithm constructing a cycle of such length.

Proof. We assume that $0<|B| \leq n-1$ and $\delta(G-B)>1$, other cases are trivial.

Consider graph $G-B$. It consists of one or more connected components. If $G$ has at least one connected component, say $H$, that is 2 -connected and is of size at least $2 \delta(G-B)$, then a cycle of length at least $\min (|V(H)|, 2 \delta(H)) \geq 2 \delta(G-B)$ can be found inside $H$ by Theorem 2 .

Now assume that each connected component in $G-B$ either contains a cut-vertex or consists of less than $2 \delta(G-B)$ vertices. Assume that there are at least two connected components in $G-B$, say $H_{1}$ and $H_{2}$. Since $\delta(G-B)>1$, both $H_{1}$ and $H_{2}$ consist of at least three vertices.

If $H_{1}$ contains a cut vertex, take one of its leaf-blocks, say $L_{1}$, and put $S_{1}=L_{1}$. Note that all vertices but one are of degree at least $\delta(G-B)$ in $L_{1}$. As $\delta(G-B)>1, L_{1}$ consists of at least three vertices and is 2-connected. If $H_{1}$ is 2-connected, put $S_{1}=H_{1}$. Find $S_{2}$ in the same way for $H_{2}$. By Menger's theorem, there are two vertex-disjoint paths from $V\left(S_{1}\right)$ to $V\left(S_{2}\right)$. Thus, there are two distinct vertices $u_{1}, v_{1} \in V\left(S_{1}\right)$ that are connected correspondingly with $u_{2}, v_{2} \in V\left(S_{2}\right)$ with two vertex-disjoint paths. Note that the total length of these paths is at least four, since the are no edges $u_{1} u_{2}$ and $v_{1} v_{2}$ in $G$. By Lemma 1 , there is a path of length at least $\delta(G-B)$ between $u_{1}$ and $v_{1}$ in $S_{1}$ if $\left|V\left(S_{1}\right)\right| \geq 4$. If $\left|V\left(S_{1}\right)\right|<4$, then $S_{1}$ is a cycle on three vertices, so there also exists a path of length at least $2 \geq\left|V\left(S_{1}\right)\right|-1 \geq \delta(G-B)$ between $u_{1}$ and $v_{1}$ in $S_{1}$. Analogously, there is a path of length at least $\delta(G-B)$ between $u_{2}$ and $v_{2}$ in $S_{2}$. Combine these two paths and the two paths outside and obtain a cycle of length at least $2 \delta(G-B)+4$ in $G$.

Now assume that there is exactly one connected component in $G-B$ of size $n-|B|$. Note that it consists of at least three vertices as $\delta(G-B)>1$. If it is 2-connected, then its size is less than $2 \delta(G-B)$. Hence, the desired cycle is obtained automatically by Theorem 1. If it is not 2-connected, take any of its cut vertices and add it to $B$ to obtain $B^{\prime}$. Note that $\delta\left(G-B^{\prime}\right) \geq \delta(G-B)-1$. Now $G-B^{\prime}$ consists of at least two connected components, so apply the discussion above for this case and obtain a cycle of length at least $2 \delta\left(G-B^{\prime}\right)+4 \geq 2 \delta(G-B)+2$.

The proof is constructive and all its steps (computing 2-connected components, finding a cutvertex, computing two vertex-disjoint paths, etc.) are implementable in polynomial time.

The similar theorem for paths can be now derived. 
Theorem 15. Let $G$ be a connected n-vertex graph. For any $B \subseteq V(G)$ there exists a simple path in $G$ of length at least $\min \{n-|B|-1,2 \delta(G-B)\}$. Moreover, there is a polynomial time algorithm constructing a path of such length.

Proof. Construct graph $G^{\prime}$ from $G$ by adding to it a universal vertex, that is the vertex adjacent to all vertices of $G$. Note that $\delta\left(G^{\prime}-B\right)=\delta(G-B)+1$ and $G^{\prime}$ consists of $n+1$ vertices. Also, $G^{\prime}$ is 2-connected, since $G$ is connected. Thus, by Theorem 14 , $G^{\prime}$ contains a simple cycle of length at least $\min \{n+1-|B|, 2 \delta(G-B)+2\}$.

If this cycle does not contain the universal vertex, this cycle is contained in $G$ as well, and we automatically obtain a path of length at least $\min \{n+1-|B|, 2 \delta(G-B)+2\}-1$ in $G$.

If the cycle contains the universal vertex, remove this vertex from the cycle. Since this vertex is incident with two edges of the cycle, we obtain a path of length at least $\min \{n+1-|B|, 2 \delta(G-B)+$ $2\}-2$ in $G$. Again, the construction can be easily turned into a polynomial time algorithm.

The following Corollary generalizes the theorem of Erdős and Gallai from [EG59, Theorem 1.16].

Corollary 3. Let $G$ be a 2-connected graph and let $s, t$ be a pair of distinct vertices in $G$. For any $B \subseteq V(G)$ there exists a path of length at least $\delta(G-B)$ between $s$ and $t$ in $G$. Moreover, there is a polynomial time algorithm constructing a cycle of such length.

Proof. Suppose that $n-|B| \geq 2 \delta(G-B)$. Use Theorem 14 to find a cycle of length at least $2 \delta(G-B)$. By Menger's theorem, there are two vertex-disjoint paths from $\{s, t\}$ to this cycle in $G$. Take these paths and the longer arc of the cycle and obtain a path of length at least $\delta(G-B)$ between $s$ and $t$.

Consider the case when $n-|B|<2 \delta(G-B)$, so $\delta(G-B) \geq(|V(G-B)|+1) / 2$. If $n-|B| \leq 3$, then $\delta(G-B) \leq 2$, so it is enough to find any path of length two between $s$ and $t$. If $n-|B| \geq 4$, then $G-B$ is 2-connected, as it contains a Hamiltonian cycle by the classical Dirac's theorem. Apply Menger's theorem to $G$ and find two vertex-disjoint paths from $\{s, t\}$ to $V(G) \backslash B$. Let these paths be a path going from $s$ to $s^{\prime} \in V(G-B)$ and from $t$ to $t^{\prime} \in V(G-B)$. By Lemma 1 , there is a path of length at least $\delta(G-B)$ between $s^{\prime}$ and $t^{\prime}$ in $G-B$. Combine this path with the paths from $s$ to $s^{\prime}$ and from $t$ to $t^{\prime}$. This yields a path of length at least $\delta(G-B)$ between $s$ and $t$ in $G$.

\section{$5 \quad$ Long $(s, t)$-Cycle}

In this section we give an FPT algorithm that finds a cycle of length at least $k$ passing through designated terminal vertices $s$ and $t$. When the length of such cycle is of order $\mathcal{O}(k)$, then the classical methods like color-coding solve the problem. The difficulty is that the length of the cycle can be arbitrarily bigger than $k$. For that case we build on the approach from [FLP ${ }^{+} 18$ ] that was used to design an algorithm for a longest $(s, t)$-path.

Now we are ready to prove Theorem 4. We restate it here.

Theorem 4. The LONG $(s, t)$-CYCLE problem is solvable in $\mathcal{O}\left((2 e)^{3 k} \cdot m n\right)$ time by a randomized Monte Carlo algorithm and in $(2 e)^{3 k} k^{\mathcal{O}(\log k)} \cdot m n \log n$ deterministic time.

Proof. Let $(G, s, t, k)$ be an instance of Long $(s, t)$-CyCle. Clearly, we can assume that $G$ is connected, because if $s$ and $t$ are in distinct connected components, then we have a trivial noinstance, and if $s$ and $t$ are in the same connected component of a disconnected graph, then we can consider the problem on the component containing $s$ and $t$ instead of $G$. To avoid additional case 
analysis, we assume that $s \neq t$. Otherwise, if $s=t$, we can do the following. If $k \leq 3$, then to solve the problem, it is sufficient to check whether $G$ has a cycle containing $s$ and this easily can be done in linear time. If $k \geq 4$, then we apply the algorithm from [FLP ${ }^{+} 18$. To be able to do it formally, we create a new vertex $t^{\prime}$ that is a false tween of $s$ and then check whether the obtained graph has an $\left(s, t^{\prime}\right)$-path of length at least $k$. Fomin, Lokshtanov, Panolan, Saurabh and Zehavi $\mathrm{FLP}^{+} 18$ ] do not state explicitly the dependency of their algorithm on the graph size. However, it can be seen that the running times of the randomized and deterministic variants of their algorithm are dominated by $\mathcal{O}\left((2 e)^{3 k} \cdot m n\right)$ and $(2 e)^{3 k} k^{\mathcal{O}(\log k)} \cdot m n \log n$, respectively. We also assume that $k \geq 4$. If $k \leq 3$, then to solve the problem, it is sufficient to find any two internally disjoint $(s, t)$-paths, and this can be done by the standard flow techniques (see, e.g., the recent textbook [Wil19]) in time $\mathcal{O}(n+m)$, because we are looking for a flow of volume 2 .

The algorithm works in two stages. First we try to find two internally vertex-disjoint $(s, t)$-paths of total length $\ell$ for $\ell \in\{k, \ldots, 3 k\}$. If such paths are found, they form the required cycle, so we stop. Otherwise, we proceed to Stage 2, where we assume that the long $(s, t)$-cycle, if it exists, is longer that $3 k$.

Stage 1. First, we check whether there are two internally disjoint $(s, t)$-paths of total length $\ell$ for some $\ell \in\{k, \ldots, 3 k\}$. For this, we apply the classical color-coding technique of Alon, Yuster, and Zwick [AYZ95]. Here the arguments are standard and we only sketch how to solve the decision version of the problem. The algorithm may be easily modified to construct the paths. We describe a randomized Monte Carlo algorithm and explain how to derandomize it in the concluding part of the theorem proof.

We color the vertices of $G$ uniformly at random by $3 k$ colors $\{1, \ldots, 3 k\}$. We say that two $(s, t)$ paths $P_{1}$ and $P_{2}$ form a colorful solution if the vertices of each of the paths have distinct colors and the colors of the internal vertices of $P_{1}$ are distinct from the colors of the internal vertices of $P_{2}$. (Clearly, the colors of $s$ and $t$ are the same in both paths.) In other words, in the $(s, t)$-cycle formed by $P_{1}$ and $P_{2}$ all vertices are colored in different colors.

We find a colorful solution by dynamic programming. Denote by $c(x)$ the color of a vertex $x \in V(G)$, and let $p=c(s)$ and $q=c(t)$. If $p=q$, then there is no colorful solution. Suppose that $p \neq q$. For a vertex $x \in V(G)$ and a nonempty set of colors $X \subseteq\{1, \ldots, 3 k\}$, define $\alpha(x, X)=$ true if there is an $(s, x)$-path $P$ with $|X|$ vertices that are colored by distinct colors from $X$, and we set $\alpha(x, X)=$ false otherwise. The values of $\alpha(x, X)$ are computed for all $x \in V(G)$ and all $X \subseteq\{1, \ldots, 3 k\}$ starting from sets of size one.

For every $x \in V(G)$ and every $i \in\{1, \ldots, 3 k\}$, we define

$$
\alpha(x,\{i\})= \begin{cases}\text { true } & \text { if } x=s \text { and } i=p \\ \text { false } & \text { otherwise. }\end{cases}
$$

Assume that $|X| \geq 2$ and the table of values of $\alpha(x, Y)$ is constructed for all $x \in V(G)$ and all $Y \subseteq\{1, \ldots, 3 k\}$ such that $|Y|<|X|$. Then for $x \in V(V)$, we set

$$
\alpha(x, X)= \begin{cases}\bigvee_{y \in N_{G}(x)} \alpha(y, X \backslash\{c(x)\}) & \text { if } c(x) \in X \\ \text { false } & \text { if } c(x) \notin X .\end{cases}
$$

By exactly the same arguments as for the color-coding algorithm for LONGEST PATH (see, e.g., $\mathrm{CFK}^{+} 15 \mathrm{~b}$, Chapter 5]), we obtain that (1) and (2) allow to compute the table of values of $\alpha(x, X)$ for all $x \in V(G)$ and all nonempty $X \subseteq\{1, \ldots, 3 k\}$ in time $\mathcal{O}\left(2^{3 k} \cdot m n\right)$, because we compute $\alpha(x, X)$ for $n$ vertices $x$ and $2^{3 k}-1$ sets $X$, and to compute $\alpha(x, X)$ using (2), we consider the neighbors of $x$. 
To complete the description of the algorithm that verifies the existence of a colorful solution, we observe that such a solution exists if and only if there are $X, Y \subseteq\{1, \ldots, 3 k\}$ such that $X \cap Y=$ $\{p, q\},|X|+|Y| \geq k+2$, and $\alpha(t, X)=\alpha(t, Y)=$ true.

Hence, it takes time $\mathcal{O}\left(2^{3 k} \cdot m n\right)$ to decide whether there is a colorful solution. If there is a colorful solution, $(G, s, t, k)$ is a yes-instance of LONG $(s, t)$-CyCLE. However, the absence of a colorful solution does not imply that we have a no-instance.

Assume that there are two internally disjoint $(s, t)$-paths $P_{1}$ and $P_{2}$ in $G$ whose total length is between $k$ and $3 k$. That is, $k \leq\left|V\left(P_{1}\right) \cup V\left(P_{2}\right)\right| \leq 3 k$. Then the probability that all vertices of $V\left(P_{1}\right) \cup V\left(P_{2}\right)$ are colored by distinct colors is at least $\frac{(3 k) !}{(3 k)^{3 k}} \geq e^{-3 k}$. The probability that there is no colorful solution is at most $1-e^{3 k}$. Therefore, by trying to find a colorful solution for $N=\left\lceil e^{3 k}\right\rceil$ random colorings, we either conclude that we have a yes-instance, or return no-answer with the mistake probability at most $\left(1-e^{3 k}\right)^{N} \leq e^{-1}$. This gives us a Monte Carlo algorithm with running time $\mathcal{O}\left((2 e)^{3 k} \cdot m n\right)$.

Stage 2. From now, we assume that we failed to solve the problem at Stage 1. This means that each solution is an $(s, t)$-cycle of length $3 k+1$. As in Stage 1 , we find two disjoint $(s, t)$-paths of total length at least $3 k+1$. This is done by generalizing the technique of Fomin, Lokshtanov, Panolan, Saurabh and Zehavi from $\left[\mathrm{FLP}^{+} 18\right]$ for finding an $(s, t)$-path of length at least $k$. Now instead of color-coding, we use the technique of random separation [CCC06]. The main step of our procedure for Stage 2 is given in Algorithm 1.

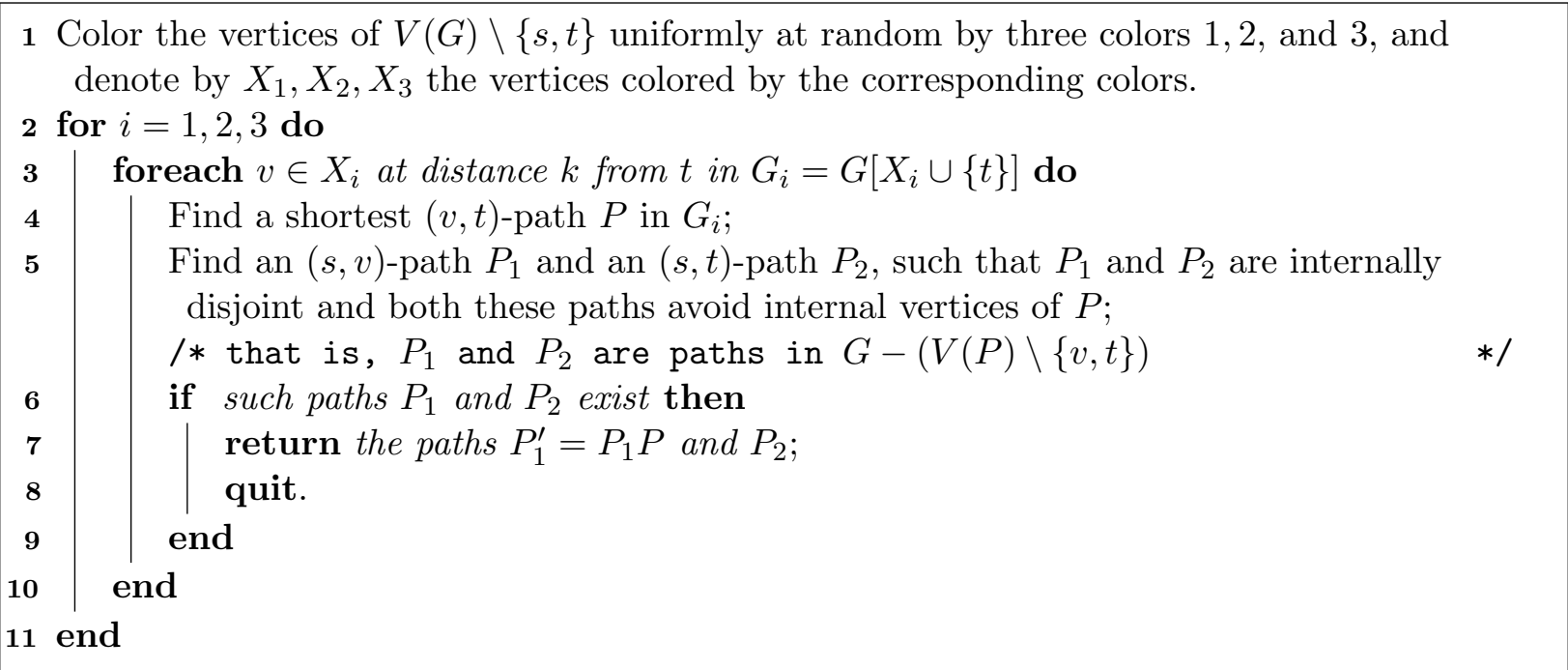

Algorithm 1: Main step of Stage 2.

Due to the conditions that $P_{1}$ does not contain internal vertices of $P$, avoids $t$, and is internally disjoint with $P_{2}$, we have that the concatenation $P_{1}^{\prime}$ of $P_{1}$ and $P$ is a path. Moreover, $P_{1}^{\prime}$ and $P_{2}$ are internally vertex disjoint $(s, t)$-paths. Since the length of $P$ is $k$, the length of $P_{1}^{\prime}$ is at least $k$. We conclude that if the algorithm returns $P_{1}^{\prime}$ and $P_{2}$, then these paths form a required $(s, t)$-cycle of length at least $k$. The algorithm runs in $\mathcal{O}(n+m)$ time, as $P_{1}$ and $P_{2}$ can be found (if they exist) by the standard flow algorithm, see e.g., Wil19].

However, the proof that the algorithm finds a solution in a yes-instance with a reasonable probability is non-trivial. It follows from the following lemma. 
Lemma 2. If $(G, s, t, k)$ is a yes-instance of LONG $(s, t)$-CYCLE, then the described algorithm finds a solution with probability at least $\frac{2}{3^{3 k-1}}$.

Proof of Lemma 2. Suppose that $(G, s, t, k)$ is a yes-instance. Then there are two internally disjoint $(s, t)$-paths $P_{1}$ and $P_{2}$ with total length at least $k$. We assume that the total length of paths $P_{1}$ and $P_{2}$ is minimum. Recall that the total length of $P_{1}$ and $P_{2}$ is at least $3 k+1$. We assume that the vertices of $P_{1}$ and $P_{2}$ are ordered in the path order starting with $s$. Thus whenever we refer to the first or the last vertices of the paths, these vertices respect this ordering. We follow the same convention for all $(x, y)$-paths, that is, we order the vertices starting from $x$. We consider two cases.

Case 1. The shortest path among $P_{1}$ and $P_{2}$ is of length at most $k$. Without loss of generality, we assume that the length of $P_{2}$ is at most $k$. Then the length of $P_{1}$ is at least $2 k+1$. Denote by $A$ the set of the first $k-1$ internal vertices of $P_{1}$, by $B$ the set of the last $k$ internal vertices of $P_{1}$, and by $C$ the set of internal vertices of $P_{2}$. Because the vertices of $V(G) \backslash\{s, t\}$ are colored uniformly at random, with probability at least $\frac{3 !}{3^{|A|} \cdot 3^{|B|} \cdot 3^{|C|}} \geq \frac{2}{3^{3 k-1}}$

(i) vertices of each of the sets $A, B$, and $C$ receive the same colors, and

(ii) vertices of distinct sets are of distinct colors.

We show that if (i) and (ii) holds, then the algorithm finds a solution. For further analysis, we assume that $A \subseteq X_{1}, B \subseteq X_{2}$, and $C \subseteq X_{3}$.

Let $v$ be the internal vertex of $P_{1}$ at distance $k$ from $t$ in the path. Then $v \in B \subseteq X_{2}$. Denote by $P_{1}^{\prime}$ the $(s, v)$-subpath of $P_{1}$. Let $P$ be an arbitrary shortest $(v, t)$-path in $G_{2}=G\left[X_{2} \cup\{t\}\right]$. Notice that $P$ is internally disjoint with $P_{2}$, because $V\left(P_{2}\right) \backslash\{s, t\} \subseteq X_{3}$, and $X_{2} \cap X_{3}=\emptyset$. We claim that $P_{1}^{\prime}$ and $P$ are internally disjoint.

Targeting towards a contradiction, assume that $V\left(P_{1}^{\prime}\right) \cap V(P) \backslash\{v\} \neq \emptyset$. Let $u$ be the first vertex of $P_{1}^{\prime}$ that is in $V(P)$. Let $Q_{1}$ be the $(s, u)$-subpath of $P_{1}^{\prime}$ and let $Q$ be the $(u, t)$-subpath of $P$. Recall that the first $k-1$ internal vertices of $P_{1}$ are in $A \subseteq X_{1}$. This implies that $A \subseteq V\left(Q_{1}\right)$. Threfore, the length of $Q_{1}$ is at least $k$. Let $\hat{P}_{1}=Q_{1} Q$. We obtain that $\hat{P}_{1}$ is an $(s, t)$-path of length at least $k$ that is internally disjoint with $P_{2}$. Hence, $\hat{P}_{1}$ and $P_{2}$ form a solution. However, the length of $\hat{P}_{1}$ is less than the length of $P_{1}$, contradicting the condition that $P_{1}$ and $P_{2}$ form a solution of minimum total length. Hence $P_{1}^{\prime}$ and $P$ are internally disjoint.

We have that $\hat{P}_{1}=P_{1}^{\prime} P$ is a path internally disjoint with $P_{2}$. We also have that $A \subseteq V\left(P_{1}\right)$ and, therefore, the length of $\hat{P}_{1}$ is at least $k$. Thus $\hat{P}_{1}$ and $P_{2}$ is a solution. By the construction of $\hat{P}_{1}$, the length of $\hat{P}_{1}$ is at most the length of $P_{1}$. Since $P_{1}$ and $P_{2}$ compose a solution of minimum total length, the length of $P_{1}$ is the same as the length of $\hat{P}_{1}$. Hence, $v$ is at distance $k$ from $v$ in $G_{3}$.

Summarizing, there is a vertex $v$ at distance $k$ from $t$ in $G_{2}$ such that for any shortest $(v, t)$-path $P$ in graph $G_{2}$, in graph $G-(V(P) \backslash\{v, t\})$ there exist an $(s, v)$-path $P_{1}$ and an $(s, t)$-path $P_{2}$ that are internally disjoint. In this case our algorithm finds a solution.

Case 2. The length of each of the paths $P_{1}$ and $P_{2}$ is at least $k+1$. Let $B$ be the last $k$ internal vertices of $P_{1}$ and let $C$ be the last $k$ internal vertices of $P_{2}$. Since each of the paths $P_{1}$ and $P_{2}$ is of length at least $k+1$ because the total length of both paths is at least $3 k+1$, we conclude the following. For some positive integers $k_{1}$ and $k_{2}$ such that $k_{1}+k_{2}=k-1$, the first $k_{1}$ internal vertices of $P_{1}$ are not in $B$, and the first $k_{2}$ internal vertices of $P_{2}$ are not in $C$. Denote by $A_{1}$ the first $k_{1}$ internal vertices of $P_{1}$ and by $A_{2}$ the first $k_{2}$ internal vertices of $P_{2}$. We set $A=A_{1} \cup A_{2}$. Thus $|A|=k-1$ and the sets $A, B$ and $C$ are pairwise disjoint. Since the vertices of $V(G) \backslash\{s, t\}$

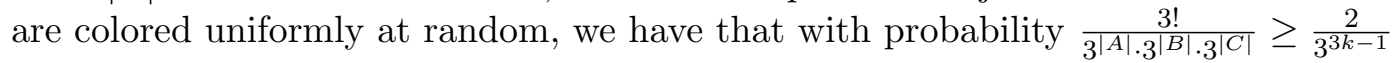


(i) vertices of each of the sets $A, B$, and $C$ are of the same color, and

(ii) vertices of distinct sets are of different colors.

As in Case 1, we show that if a coloring satisfies (i) and (ii), then the algorithm finds a solution. Without loss of generality, we assume that $A \subseteq X_{1}, B \subseteq X_{2}$, and $C \subseteq X_{3}$.

Let $v_{1}$ be the internal vertex of $P_{1}$ at distance $k$ from $t$ in $P_{1}$, and let $v_{2}$ be the internal vertex of $P_{2}$ at distance $k$ from $t$ in $P_{2}$. Note that $v_{1} \in B \subseteq X_{2}$ and $v_{2} \in C \subseteq X_{3}$. Denote by $P_{1}^{\prime}$ the $\left(s, v_{1}\right)$ subpath of $P_{1}$ and by $P_{1}^{\prime \prime}$ the $\left(v_{1}, t\right)$-subpath of $P_{1}$. Similarly, we define $P_{2}^{\prime}$ as the $\left(s, v_{2}\right)$-subpath of $P_{2}$ and $P_{2}^{\prime \prime}$ as the $\left(v_{2}, t\right)$-subpath of $P_{2}$.

We prove the following claim.

Claim 1. At least one the following options holds.

- Either for every shortest $\left(v_{1}, t\right)$-path $Q_{1}$ in $G_{2}=G\left[X_{2} \cup\{t\}\right]$, paths $Q_{1}$ and $P_{2}$ are internally disjoint,

- or for every shortest $\left(v_{2}, t\right)$-path $Q_{2}$ in $G_{3}=G\left[X_{3} \cup\{t\}\right]$, paths $Q_{2}$ and $P_{1}$ are internally disjoint.

Proof of Claim 1. The proof is by contradiction. Assume that there is a shortest $\left(v_{1}, t\right)$-path $Q_{1}$ in $G_{2}$ and a shortest $\left(v_{2}, t\right)$-path $Q_{2}$ in $G_{3}$ such that neither $Q_{1}$ and $P_{2}$ are internally disjoint, nor $Q_{2}$ and $P_{1}$ are internally disjoint. See Figure 2 .

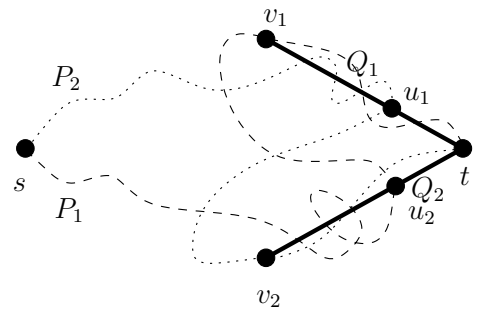

a)

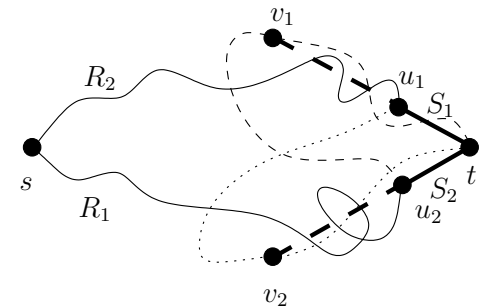

b)

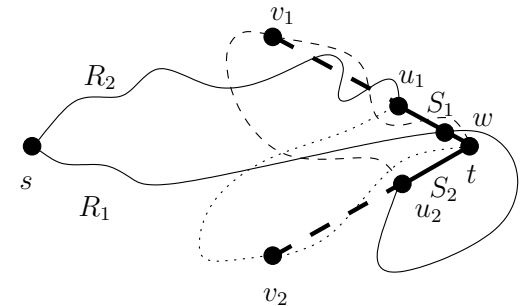

c)

Figure 2: Structure of the paths $P_{1}, P_{2}, Q_{1}$, and $Q_{2}$. a) A dashed line shows path $P_{1}$, a dotted line indicates $P_{2}$, and solid lines are used for $Q_{1}$ and $Q_{2}$. b) Solid lines indicate paths $R_{1}, R_{2}, S_{1}$, and $S_{2}$. The thin lines are used for $R_{1}$ and $R_{2}$, while the thick lines for $S_{1}$ and $S_{2}$. The choice of $w$ is demonstrated in c).

Notice that $Q_{1}$ and $Q_{2}$ are internally disjoint since they are paths in $G_{2}$ and $G_{3}$ respectively, and $t$ is the unique common vertex of these graphs. Let $u_{1}$ be the vertex of $V\left(Q_{1}\right) \cap V\left(P_{2}\right)$ distinct from $t$ that is at the minimum distance from $t$ in $Q_{1}$. Similarly, let $u_{2}$ be the vertex of $V\left(Q_{2}\right) \cap V\left(P_{1}\right)$ distinct from $t$ that is at the minimum distance from $t$ in $Q_{2}$. The choice of $u_{1}$ and $u_{2}$ is shown in Figure 2 (a). Because $V\left(P_{2}^{\prime \prime}\right) \subseteq X_{3}$ and $V\left(P_{1}^{\prime \prime}\right) \subseteq X_{2}$, we have that $u_{1} \in V\left(P_{2}^{\prime}\right)$ and $u_{2} \in V\left(P_{1}^{\prime}\right)$. Denote by $R_{1}$ the $\left(s, u_{2}\right)$-subpath of $P_{1}$ and by $R_{2}$ the $\left(s, u_{1}\right)$-subpath of $P_{2}$. Let also $S_{1}$ be the $\left(u_{1}, t\right)$-subpath of $Q_{1}$ and $S_{2}$ be the $\left(u_{2}, t\right)$-subpath of $Q_{2}$. The construction of these paths is shown in Figure 2 (b).

We claim that paths $S_{1}$ and $R_{1}$ have no common vertices. For the sake of contradiction, let $V\left(S_{1}\right) \cap V\left(R_{1}\right) \neq \emptyset$ and assume that $w$ is the first vertex of $R_{1}$ in $V\left(S_{1}\right)$ (see Figure 2 (c)). Since $R_{1}$ and $R_{2}$ are internally vertex disjoint, $w$ is an internal vertex of $S_{1}$. By the choice of $u_{1}$, there is no internal vertex of $S_{1}$ that belongs to $P_{2}$. Hence, the concatenation $\hat{P}_{1}$ of the $(s, w)$-subpath of $R_{1}$ and the $(w, t)$-subpath of $S_{1}$, gives a path that is internally vertex disjoint with $P_{2}$. Observe 
also that $A_{1} \subseteq V\left(\hat{P}_{1}\right)$, because $w \in B$ and the first $k_{1}$ internal vertices of $P_{1}$ are in $A_{1}$. Therefore, the total length of paths $\hat{P}_{1}$ and $P_{2}$ is at least $k$. However, the length of the $(s, w)$-subpath of $R_{1}$ is less than the length of $P_{1}^{\prime}$ and the length of the $(w, t)$-subpath of $S_{1}$ is less than $k$. Therefore, the length of $\hat{P}_{1}$ is less than the length of $P_{1}$, contradicting the choice of $P_{1}$ and $P_{2}$.

This proves that $S_{1}$ and $R_{1}$ have no common vertices. By the same arguments, $S_{2}$ and $R_{2}$ also have no common vertices.

Consider $(s, t)$-paths $\hat{P}_{1}=R_{2} S_{1}$ and $\hat{P}_{2}=R_{1} S_{2}$. Since paths $S_{1}$ and $R_{1}$ do not intersect and paths $S_{2}$ and $R_{2}$ also do not intersect, we have that paths $\hat{P}_{1}$ and $\hat{P}_{2}$ are internally disjoint. Because $A_{1} \subseteq V\left(\hat{P}_{2}\right)$ and $A_{2} \subseteq V\left(\hat{P}_{1}\right)$, the total length of paths $\hat{P}_{1}$ and $\hat{P}_{2}$ is at least $k$. However, because the length of $P_{1}^{\prime \prime}$ is less than the length of $S_{1}$ and because the length of $P_{2}^{\prime \prime}$ is less than the length of $S_{2}$, the total length of $\hat{P}_{1}$ and $\hat{P}_{2}$ is less than the total length of $P_{1}$ and $P_{2}$. This contradict the choice of $P_{1}$ and $P_{2}$ and proves the claim.

By Claim 1, without loss of generality, we assume that for every shortest $\left(v_{1}, t\right)$-path $Q_{1}$ in $G_{2}$, paths $Q_{1}$ and $P_{2}$ are internally disjoint. Now we repeat the arguments from Case 1 . We observe that every shortest $\left(v_{1}, t\right)$-path $Q_{1}$ in $G_{2}$ is internally disjoint with $P_{1}^{\prime}$. Indeed, if this is not the case, we can select the first vertex $u$ of $P_{1}^{\prime}$ that is in $Q_{1}$. Then by replacing $P_{1}$ by the concatenation of the $(s, u)$-subpath of $P_{1}^{\prime}$ and the $(u, t)$-subpath of $Q_{1}$, we obtain a solution with a shorter total length. But this contradicts the choice of $P_{1}$ and $P_{2}$. Since $Q_{1}$ and $P_{1}^{\prime}$ are internally vertex disjoint, we have that the cycle formed by paths $\hat{P}_{1}=P_{1}^{\prime} Q_{1}$ and $P_{2}$ is a solution. This implies that $Q_{1}$ and $P_{1}^{\prime \prime}$ have the same length. Therefore $v_{1}$ is at distance $k$ from $t$.

We conclude that there is $v_{1}$ at distance $k$ from $t$ in $G_{2}$ such that for every shortest $(v, t)$-path $Q_{1}$ in $G_{2}$, there are an $\left(s, v_{1}\right)$-path $P_{1}^{\prime}$ and an $(s, t)$-path $P_{2}$ in $G-(V(P) \backslash\{v, t\})$ that are internally disjoint. Then the algorithm finds a solution. This concludes Case 2 and the proof of Lemma 2 .

By Lemma 2, if we iterate Algorithm $133^{3 k-1} / 2$ times, then we either find a solution, or return the no-answer with the error probability at most $\left(1-\frac{2}{3^{3 k-1}}\right)^{3^{3 k-1} / 2} \leq e^{-1}$. Thus we have a Monte Carlo algorithm with false negatives that runs in time $\mathcal{O}\left(3^{3 k} \cdot(n+m)\right)$.

Derandomization. For the Monte Carlo algorithm that we use in the first stage (finding a short cycle), derandomization uses the standard technique. We replace random colorings by functions from the $(n, 3 k)$-perfect hash family of functions of $\operatorname{size} e^{3 k} k^{\mathcal{O}(\log k)} \cdot \log n$ that can be constructed in time $e^{3 k} k^{\mathcal{O}(\log k)} \cdot n \log n$ by the results of Naor, Schulman, and Srinivasan [NSS95] (we refer to $\left[\mathrm{CFK}^{+} 15 \mathrm{~b}\right.$, Chapter 5] for the detailed introduction to the technique). This allows us to check in $(2 e)^{3 k} k^{\mathcal{O}(\log k)} \cdot m n \log n$ deterministic time whether there are two internally vertex disjoint $(s, t)$ paths in $G$ whose total length is at least $k$ but at most $3 k$.

To derandomize the algorithm from the second stage that uses random separation, we have to do an extra work. This is because commonly random separation is used to distinguish two sets $\mathrm{CFK}^{+} 15 \mathrm{~b}$, Chapter 5]. In our algorithm we distinguish three sets; derandomization here is slightly different and is based on Lemma 3 . Lemma 3 could be a folklore, but we did not find it in the literature and prove it here for completeness.

Let $k$ and $n$ be positive integers. An $(n, k)$-universal set is a family $\mathcal{U}$ of subsets of $\{1, \ldots, n\}$ such that for any $S \subseteq\{1, \ldots, n\}$ of size $k$, the family $\{A \cap S \mid A \in \mathcal{U}\}$ contains all $2^{k}$ subsets of $S$. We use the following result of Naor, Schulman, and Srinivasan [NSS95].

Proposition 1 ([NSS95]). For any $n, k \geq 1$, one can construct an $(n, k)$-universal set of size $2^{k} k^{\mathcal{O}(\log k)} \cdot \log n$ in time $2^{k} k^{\mathcal{O}(\log k)} \cdot n \log n$.

Using Proposition 1, we prove the following lemma. 
Lemma 3. For an $n$-element set $\Omega$ and a positive $k$, there is a family of functions $\mathcal{F}_{n, k}$ mapping $\Omega$ to $\{1,2,3\}$ of size $2^{5 k} k^{\mathcal{O}(\log k)} \cdot(\log n)^{2}$ such that for every triple of disjoint nonempty sets $A_{1}, A_{2}, A_{3} \subseteq$ $\Omega$, each of size at most $k$, there is $f \in \mathcal{F}_{n, k}$ with the property that

- $f(x)=f(y)$ if $x, y \in A_{i}$ for some $i \in\{1,2,3\}$,

- $f(x) \neq f(y)$ if $x \in A_{i}$ and $y \in A_{j}$ for distinct $i, j \in\{1,2,3\}$.

Moreover, $\mathcal{F}_{n, k}$ can be constructed in $2^{5 k} k^{\mathcal{O}(\log k)} \cdot n^{2} \log n$ time.

Proof. If $n \leq 3 k$, then we define $\mathcal{F}_{n, k}$ to be the family of all at most $3^{3 k}$ mappings $f: \Omega \rightarrow\{1,2,3\}$. Hence, from now we assume that $n \geq 3 k$. Let $\Omega=\left\{\omega_{1}, \ldots, \omega_{n}\right\}$.

We apply Proposition 1 to construct the following family of universal sets. We construct an $(n, 3 k)$ universal set $\mathcal{U}^{(1)}$. Then for every positive $p \leq n$, we construct an $(p, 2 k)$-universal set $\mathcal{U}_{p}^{(2)}$. Then $\mathcal{F}_{n, k}$ is constructed as follows. For every $U=\left\{i_{1}, \ldots, i_{p}\right\} \in \mathcal{U}^{(1)}$ and every $W=\left\{j_{1}, \ldots, j_{q}\right\} \in$ $\mathcal{U}_{p}^{(2)}$, we construct $f: \Omega \rightarrow\{1,2,3\}$ such that for every $h \in\{1, \ldots, n\}$,

$$
f\left(\omega_{h}\right)= \begin{cases}1 & \text { if } h \notin\left\{i_{1}, \ldots, i_{p}\right\} \\ 2 & \text { if } h \in\left\{i_{1}, \ldots, i_{p}\right\} \backslash\left\{i_{j_{1}}, \ldots, i_{j_{q}}\right\} \\ 3 & \text { if } h \in\left\{i_{j_{1}}, \ldots, i_{j_{q}}\right\} .\end{cases}
$$

To see that $\mathcal{F}_{n, k}$ satisfies the required property, consider arbitrary disjoint sets $A_{1}, A_{2}, A_{3} \subseteq \Omega$ of size at most $k$. We assume without loss of generality that each $A_{i}$ is of size exactly $k$ (otherwise, we can complement the sets by adding elements of $\Omega$ that are outside these sets). Let $A_{i}=$ $\left\{\omega_{i_{1}^{i}}, \ldots, \omega_{i_{k}^{i}}\right\}$ for $i \in\{1,2,3\}$. Let $S=\left\{i_{1}^{1}, \ldots, i_{k}^{1}\right\} \cup\left\{i_{1}^{2}, \ldots, i_{k}^{2}\right\} \cup\left\{i_{1}^{3}, \ldots, i_{k}^{3}\right\}$. By definition, the $(n, 3 k)$-universal set $\mathcal{U}^{(1)}$, contains a set $X$ such that $S \cap X=\left\{i_{1}^{2}, \ldots, i_{k}^{2}\right\} \cup\left\{i_{1}^{3}, \ldots, i_{k}^{3}\right\}$. Let $p=|X|$ and assume that $X=\left\{j_{1}, \ldots, j_{p}\right\}$. Again by definition, the $(p, 2 k)$-universal set $\mathcal{U}_{p}^{(2)}$ contains a set $Z$ such that for every $s \in Z, j_{s} \neq i_{1}^{2}, \ldots, i_{k}^{2}$, and for every $t \in\{1, \ldots, k\}$, there is $s \in Z$ such that $j_{s}=i_{t}^{2}$. This implies that for $f \in \mathcal{F}_{n, k}$ constructed for $X \in \mathcal{U}^{(1)}$ and $Y \in \mathcal{U}_{p}^{(2)}, f(x)=i$ if $x \in A_{i}$ for $i \in\{1,2,3\}$. Therefore, $f$ distinguishes the sets $A_{1}, A_{2}, A_{3}$.

By Proposition 1, $\left|\mathcal{U}^{(1)}\right|=2^{3 k} k^{\mathcal{O}(\log k)} \cdot \log n$ and $\left|\mathcal{U}_{p}^{(2)}\right|=2^{2 k} k^{\mathcal{O}(\log k)} \cdot \log n$. Therefore, $\left|\mathcal{F}_{n, k}\right| \leq$ $2^{5 k} k^{\mathcal{O}(\log k)}(\log n)^{2}$. By Proposition 1, the universal sets can be constructed in time $2^{3 k} k^{\mathcal{O}(\log k)}$. $n^{2} \log n$. Then we construct $\mathcal{F}_{n, k}$ in time $2^{5 k} k^{\mathcal{O}(\log k)} \cdot n(\log n)^{2}$.

To derandomize our algorithm, we apply Lemma 3. Notice that the only property of random colorings that we use in the algorithm is that with sufficiently high probability the sets $A, B$, and $C$ defined in the proof of Lemma 2 are colored by distinct colors. The sets $A, B$, and $C$ have sizes at most $k$, and they are subsets of $V(G) \backslash\{s, t\}$. This implies that the random colorings can be replaced by functions of the family $\mathcal{F}_{n-2, k}$ for $\Omega=V(G) \backslash\{s, t\}$. Since Algorithm 1 runs in $\mathcal{O}(n+m)$ time, the running time is $2^{5 k} k^{\mathcal{O}(\log k)} \cdot(n+m)(\log n)^{2}$. Taking into account the time for constructing $\mathcal{F}_{n-2, k}$, we conclude that the problem can be solved in $2^{5 k} k^{\log k} \cdot m n \log n$ deterministic time.

Recall that in the first stage of our algorithm, we try to find two internally disjoint $(s, t)$ paths of total length $\ell$ for some $\ell \in\{k, \ldots, 3 k\}$, and this can be done in $(2 e)^{3 k} \cdot m n$ randomized and $(2 e)^{3 k} k^{\mathcal{O}(\log k)} \cdot m n \log n$ deterministic time. Since $(2 e)^{3} \geq 2^{5} \geq 3^{3}$ and $n m \geq n(n-1)$ as $G$ is assumed to be connected, we obtain that the running time of the first stage dominates the running time of the second. We conclude that the problem can be solved in $(2 e)^{3 k} \cdot m n$ randomized and $(2 e)^{3 k} k^{\mathcal{O}(\log k)} \cdot m n \log n$ deterministic time. It is plausible that the running time for the first stage can be improved by making use of more sophisticated techniques for LONGEST PATH and 
Longest CyCle (see, e.g., [FLPS16, Zeh16]) but such an improvement goes beyond the scope of our paper.

\section{Long Erdős-Gallai $(s, t)$-Path}

In this section we prove Theorem 5. Long ERDös-Gallai $(s, t)$-PATH is solvable in time $2^{\mathcal{O}(k+|B|)} \cdot n^{\mathcal{O}(1)}$. The proof of the theorem relies on the structural properties of graphs with a long path. The notions of Erdös-Gallai decomposition and Erdös-Gallai component are crucial here. We prove several combinatorial and algorithmic properties of Erdős-Gallai decomposition, and then apply the obtained properties in the proof of Theorem 5 .

\subsection{Erdős-Gallai decompositions and structures}

We need to introduce the operation of B-refinements. The intuition behind this operation is the following. In our proof, we will be using the following rerouting strategy. Suppose we have an $(s, t)$-path $P$, and we want to construct a longer path by rerouting some parts of $P$ through a connected component $H$ of $G-V(P)$. If $H$ is 2-connected, we can try to apply Theorem 2 to argue that such an enlargement of $P$ is possible. However, when $H$ is not 2-connected, we want to eliminate some "insignificant" parts of $H$. While in the refinement we contract some of the edges inside $H$, all edges between $H$ and the remaining part of the graph remain.

Definition 1 ( $B$-refinement of $H$ ). Let $H$ be a connected subgraph of a graph $G$ and $B \subset V(G)$. The $B$-refinement of $H$, denoted by $R_{B}(H)$, is the graph obtained by the following process. Start with $R_{B}(H):=G$. While $H$ is not 2-connected and contains a leaf-block with all inner vertices from $B$, contract all edges in $H$ from this leaf-block to its cut-vertex.

In other words, $R_{B}(H)$ is obtained from $G$ by repeatedly contracting edges of $H$ from the leaf-blocks of $H$ whose inner vertices are from $B$. Note that in $B$-refinement only edges with both endpoints in $B$ can be contracted. We also say that $R_{B}(H)$ is obtained from $G$ by applying $B$-refinement to $H$.

We are ready to introduce the primary tool for solving LONG ERDős-GALLAI $(s, t)$-PATH. This structure arises in the extremal cases when we cannot enlarge an $(s, t)$-path by local replacement used in the proof of the Erdős-Gallai's theorem. This is where the name we use for the decomposition comes from.

Definition 2 (Erdős-Gallai decomposition and Erdős-Gallai component). Let $P$ be a path in a 2-connected graph $G$ and let $B \subseteq V(G)$. We say that two disjoint paths $P_{1}$ and $P_{2}$ in $G$ induce an Erdös-Gallai decomposition for $P$ and $B$ in $G$ if

- Path $P$ is of the form $P=P_{1} P^{\prime} P_{2}$, where the inner path $P^{\prime}$ has at least $\delta(G-B)$ edges.

- Let $G^{\prime}$ be the graph obtained from $G$ by applying $B$-refinement to every connected component $H$ of $G-V\left(P_{1} \cup P_{2}\right)$, except those components $H$ with $V(H) \subseteq B$. Note that no edges of the paths $P_{1}$ and $P_{2}$ are contracted. There are at least two connected components $H^{\prime}$ in $G^{\prime}-V\left(P_{1} \cup P_{2}\right)$ with $V\left(H^{\prime}\right) \nsubseteq B$. For every such connected component $H^{\prime}$ holds $\left|V\left(H^{\prime}\right)\right| \geq 3$ and one of the following.

(R1) $H^{\prime}$ is 2-connected and the maximum size of a matching in $G^{\prime}$ between $V\left(H^{\prime}\right)$ and $V\left(P_{1}\right)$ is one, and between $V\left(H^{\prime}\right)$ and $V\left(P_{2}\right)$ is also one; 


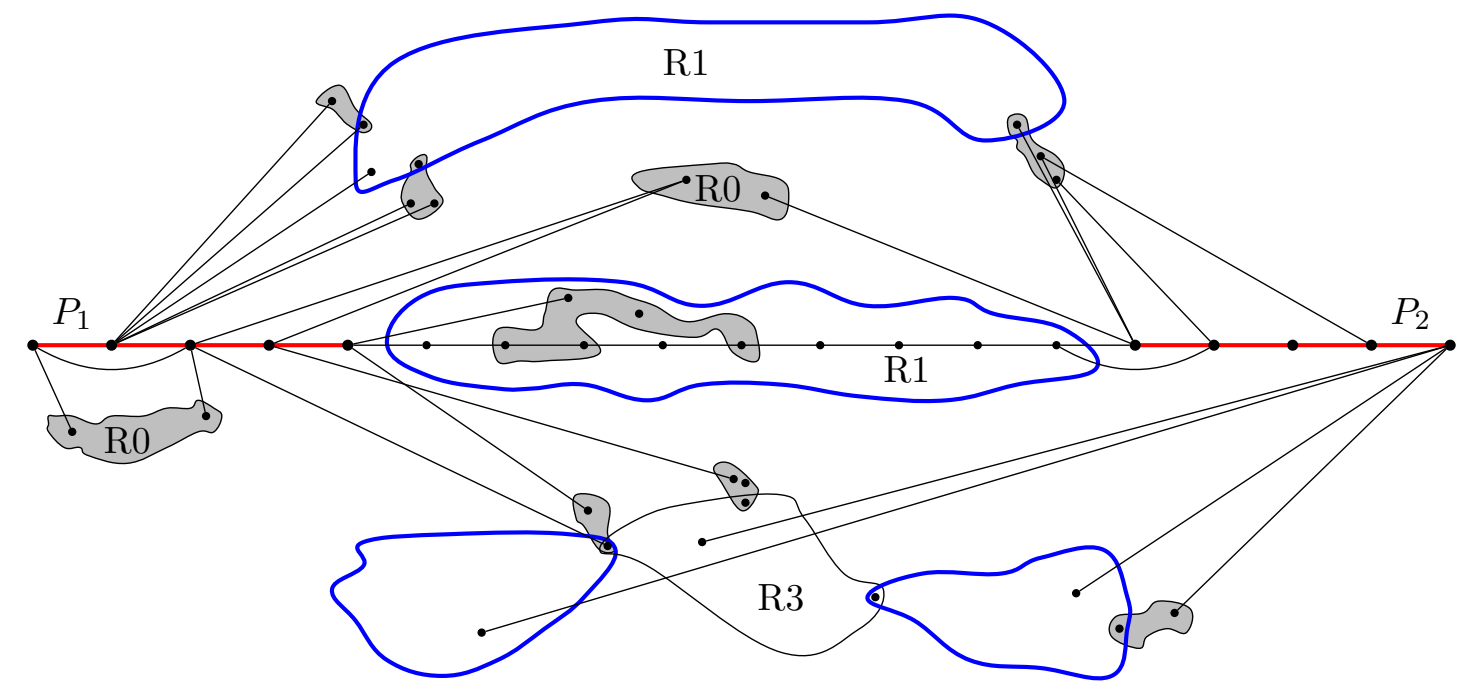

Figure 3: A schematic example of an Erdös-Gallai decomposition for a path. The components are denoted by their respective types in the decomposition, R0 denotes components consisting entirely of vertices from $B$ (marked by light gray). The four Erdős-Gallai components are marked by thick blue borders.

(R2) $H^{\prime}$ is not 2-connected, exactly one vertex of $P_{1}$ has neighbors in $H^{\prime}$, that is $\mid N_{G^{\prime}}\left(V\left(H^{\prime}\right)\right) \cap$ $V\left(P_{1}\right) \mid=1$, and no inner vertex from a leaf-block of $H^{\prime}$ has a neighbor in $P_{2}$;

(R3) The same as (R2), but with $P_{1}$ and $P_{2}$ interchanged. That is, $H^{\prime}$ is not 2-connected, $\left|N_{G^{\prime}}\left(V\left(H^{\prime}\right)\right) \cap V\left(P_{2}\right)\right|=1$, and no inner vertex from a leaf-block of $H^{\prime}$ has a neighbor in $P_{1}$.

The set of Erdős-Gallai components for an Erdős-Gallai decomposition is defined as follows. First, for each component $H^{\prime}$ of type (R1), $H^{\prime}$ is an Erdős-Gallai component of the Erdős-Gallai decomposition. Second, for each $H^{\prime}$ of type (R2), or of type (R3), all its leaf-blocks are also Erdős-Gallai components of the Erdős-Gallai decomposition. The example of an Erdös-Gallai decomposition is given in Figure 3 .

The following lemma provides a polynomial time algorithm that either finds a long path in the given graph or constructs an Erdős-Gallai decomposition.

Lemma 4. Let $G$ be a 2-connected graph with two distinct vertices $s$ and $t, B \subseteq V(G)$ be a subset of vertices such that $s, t \in B$, and $k>0$ be an integer such that $4 k+2|B|+4 \leq \delta(G-B)$. There is a polynomial time algorithm that

- either outputs an $(s, t)$-path $P$ of length at least $\delta(G-B)+k$,

- or outputs an $(s, t)$-path $P$ with $V(P) \cup B=V(G)$,

- or outputs an $(s, t)$-path $P$ with paths $P_{1}, P_{2}$ that induce an Erdös-Gallai decomposition for $P$ and $B$ in $G$.

Proof. By Corollary 3, an $(s, t)$-path $P$ of length at least $\delta(G-B)$ can be found in polynomial time. If the length of $P$ is at least $\delta(G-B)+k$, we output it and stop. Otherwise, we try to make $P$ longer by replacing some of its parts with paths in $G-V(P)$. 
We first contract some edges of $G$ in a way similar to the definition of Erdös-Gallai decompositions. For each connected component $H$ of $G-V(P)$ such that $V(H)$ is not in $B$, we perform $B$-refinement of $H$. That is, while $H$ is not 2-connected and has a leaf-block with all inner vertices from $B$, we contract all edges of this leaf-block. We denote the resulting graph by $G^{\prime}$. Note that $G^{\prime}$ still contains $P$ as a subgraph and that $\delta\left(G^{\prime}-B\right) \geq \delta(G-B)$. If we find an $(s, t)$-path that is longer than $P$ in $G^{\prime}$, this path can be easily transformed into a path of the same or greater length in $G$. Moreover, if we find paths $P_{1}$ and $P_{2}$ that induce an Erdős-Gallai decomposition for $P$ in $G^{\prime}$, then $P_{1}, P_{2}$ induce an Erdös-Gallai decomposition for $P$ in graph $G$ as well. Thus, from now on, we proceed with the graph $G^{\prime}$.

We start with the trivial case. If $V\left(G^{\prime}\right) \backslash P \subseteq B$, then $V(P) \cup B=V(G)$. Hence, the algorithm just outputs $P$ and stops.

From now on we assume that $\left(V\left(G^{\prime}\right) \backslash B\right) \backslash V(P) \neq \emptyset$. Let $H^{\prime}$ be a connected component in $G^{\prime}-V(P)$ that contains at least one vertex in $V\left(G^{\prime}\right) \backslash B$. We consider several cases. The first case is a trivial case when $H^{\prime}-B$ contains at most two vertices. The second case corresponds to Erdős-Gallai components of type (R1), while the third case to Erdös-Gallai components of types (R2) and (R3).

If we find out that $P$ can be enlarged, we replace $P$ with the longer path in $G$ and start trying to make it longer again. Throughout the proof and all its claims, we consider that $P$ cannot be made longer with the replacement operation.

Case 1: $H^{\prime}-B$ contains at most two vertices. In this case, each vertex in $V\left(H^{\prime}-B\right)$ has at least $\delta\left(G^{\prime}-B\right)-2$ neighbors in $P$. If the length of $P$ is less than $2 \delta(G-B)-4 \geq \delta(G-B)+4 k$, then each vertex in $V\left(H^{\prime}-B\right)$ has two consecutive vertices in $P$ as neighbors. Hence, any such vertex can be inserted in $P$ between such two neighbors, so the length of $P$ increases by one.

Conclusion of Case 1. Either $H^{\prime}-B$ consists of at least three vertices, or the length of $P$ can be increased (in polynomial time).

Case 2: $H^{\prime}$ is 2-connected. We start with the following claim.

Claim 2. If there is a matching of size at least three between $V\left(H^{\prime}\right)$ and $V(P)$ in $G^{\prime}$, then the length of $P$ can be enlarged in polynomial time.

Proof of Claim 2. As $\delta\left(G^{\prime}-B\right) \geq \delta(G-B), 2 \delta\left(G^{\prime}-B\right)-2>\delta(G-B)+4 k+2|B|>\delta(G-B)+k$. So we assume that the length of $P$ is at most $2 \delta\left(G^{\prime}-B\right)-1$. Let $u_{1} v_{1}, u_{2} v_{2}, u_{3} v_{3}$ be a matching in $G^{\prime}$ such that $u_{1}, u_{2}, u_{3} \in V\left(H^{\prime}\right)$ and $v_{1}, v_{2}, v_{3} \in V(P)$.

If no vertex in $V\left(H^{\prime}-B\right)$ has a neighbor in $P$, then $\delta\left(H^{\prime}-B\right) \geq \delta\left(G^{\prime}-B\right)$. By Corollary 3 , there is a path of length at least $\delta\left(G^{\prime}-B\right)$ between any pair of vertices in $H^{\prime}$. Because the length of $P$ is at most $2 \delta\left(G^{\prime}-B\right)-1<2 \delta\left(G^{\prime}-B\right)+4$, at least for one pair $\left\{v_{i}, v_{j}\right\}, i \neq j$, the distance between $v_{i}$ and $v_{j}$ in $P$ is less than $\delta\left(G^{\prime}-B\right)+2$. Then we replace the $\left(v_{i}, v_{j}\right)$-subpath in $P$ with the path $v_{i} u_{i} \rightsquigarrow u_{j} v_{j}$, where $u_{i} \rightsquigarrow u_{j}$ is a path between $u_{i}$ and $u_{j}$ in $H^{\prime}$ of length at least $\delta\left(G^{\prime}-B\right)$. The length of $v_{i} u_{i} \rightsquigarrow u_{j} v_{j}$ is at least $\delta\left(G^{\prime}-B\right)+2$ and hence we can enlarge $P$.

Now we assume that there is at least one vertex $w \in V\left(H^{\prime}-B\right)$ with a neighbor in $P$. We can assume that in the matching $u_{1} v_{1}, u_{2} v_{2}, u_{3} v_{3}$, one of the vertices $u_{i}=w$. (If all $u_{i} \in B$, we just replace $u_{1}$ with $w$.) Vertex $w$ has at least $\max \left\{1, \delta\left(G^{\prime}-B\right)-\delta\left(H^{\prime}-B\right)\right\}$ neighbors in $P$. Let $S$ be the set of neighbors of $u_{1}, u_{2}, u_{3}$ in $P$, that is, $S:=\left(N_{G^{\prime}}\left(u_{1}\right) \cup N_{G^{\prime}}\left(u_{2}\right) \cup N_{G^{\prime}}\left(u_{3}\right)\right) \cap V(P)$. Then the size of $S$ is at least $\max \left\{\delta\left(G^{\prime}-B\right)-\delta\left(H^{\prime}-B\right), 3\right\}$. Let $s_{1}, s_{2}, \ldots, s_{|S|}$, be the order of vertices from $S$ in the path $P$. If the length of one of the subpaths $s_{i}, s_{i+1}, i \in\{1, \ldots,|S|-1\}$, of $P$ is 1 , we can enlarge $P$ by replacing $s_{i}, s_{i+1}$ with an $\left(s_{i}, s_{i+1}\right)$-path of length at least 2 going through $V\left(H^{\prime}\right)$. Moreover, at least two of these subpaths go between neighbors of $u_{i}$ and $u_{j}$ for distinct $i$ and $j$. If one of these paths, say between $s_{\ell}$ and $s_{\ell+1}$, is of length less than $\delta\left(H^{\prime}-B\right)+2$, we can 
increase $P$ by replacing it with path $s_{\ell} u_{i} \rightsquigarrow u_{j} s_{\ell+1}$, where $u_{i} \rightsquigarrow u_{j}$ is a path between $u_{i}$ and $u_{j}$ in $H^{\prime}$ of length at least $\delta\left(H^{\prime}-B\right)$. This means that if we cannot enlarge $P$, then the length of $P$ is at least $2(|S|-3)+2\left(\delta\left(H^{\prime}-B\right)+2\right) \geq 2\left(\delta\left(G^{\prime}-B\right)-\delta\left(H^{\prime}-B\right)-3\right)+2\left(\delta\left(H^{\prime}-B\right)+2\right)=$ $2 \delta\left(G^{\prime}-B\right)-2>\delta(G-B)+k$.

By the claim and the fact that $G$ is 2-connected, we can assume that the maximum size of a matching between $V\left(H^{\prime}\right)$ and $V(P)$ in $G^{\prime}$ is exactly two.

Claim 3. There is a path of length at least $\delta\left(G^{\prime}-B\right)-2$ between any pair of vertices in $H^{\prime}$.

Proof of Claim 3. Let $h_{1} v_{1}, h_{2} v_{2}$ be the edges of the maximum matching between $V\left(H^{\prime}\right)$ and $V(P)$ in $G^{\prime}$, where $h_{1}, h_{2} \in V\left(H^{\prime}\right), v_{1}, v_{2} \in V(P)$. Note that no vertex in $V\left(H^{\prime}\right) \backslash\left\{h_{1}, h_{2}\right\}$ has neighbours in $V(P) \backslash\left\{v_{1}, v_{2}\right\}$.

If $h_{1}$ and $h_{2}$ have no neighbours other than $v_{1}$ and $v_{2}$ in $V(P)$, then, trivially, $N_{G}\left(V\left(H^{\prime}\right)\right) \cap$ $V(P)=\left\{v_{1}, v_{2}\right\}$, so $\delta\left(H^{\prime}-B\right) \geq \delta\left(G^{\prime}-B\right)-2$.

Without loss of generality, we now assume that $h_{1}$ has a neighbour $v_{3} \in V(P) \backslash\left\{v_{1}, v_{2}\right\}$. Then no vertex in $V\left(H^{\prime}\right) \backslash\left\{h_{1}, h_{2}\right\}$ can have $v_{1}$ as a neighbour. Analagously, if $h_{2}$ has a neighbour other than $v_{1}$ or $v_{2}$, no vertex in $V\left(H^{\prime}\right) \backslash\left\{h_{1}, h_{2}\right\}$ can have $v_{2}$ as a neighbour. Hence, if $N_{G}\left(h_{i}\right) \nsubseteq\left\{v_{1}, v_{2}\right\}$ for both $i=1$ and $i=2$, then $\delta\left(H^{\prime}-\left(B \cup\left\{h_{1}, h_{2}\right\}\right)\right) \geq \delta\left(G^{\prime}-B\right)-2$.

We now assume that $h_{2}$ has no neighbours other than $v_{1}$ and $v_{2}$ in $V(P)$. If $h_{2}$ is adjacent to $v_{1}$, then no vertex in $V\left(H^{\prime}\right) \backslash\left\{h_{1}, h_{2}\right\}$ can be adjacent to $v_{2}$, as we would obtain a matching $h_{1} v_{3}, h_{2} v_{1}$, $h_{3} v_{2}$ of size at least three. Hence, if $h_{2} v_{1} \in E\left(G^{\prime}\right)$, then $\delta\left(H^{\prime}-\left(B \cup\left\{h_{1}, h_{2}\right\}\right)\right) \geq \delta\left(G^{\prime}-B\right)-2$. If $h_{2}$ is not adjacent to $v_{1}$, then all vertices in $V\left(H^{\prime}\right) \backslash\left\{h_{1}\right\}$ only can have $v_{2}$ as a neighbour, so $\delta\left(H^{\prime}-\left(B \cup\left\{h_{1}\right\}\right)\right) \geq \delta\left(G^{\prime}-\left(B \cup\left\{h_{1}\right\}\right)\right)-1 \geq \delta\left(G^{\prime}-B\right)-2$.

It is left to apply Corollary 3 to all of the cases.

Hence, if there is a matching between $V\left(H^{\prime}\right)$ and two vertices on $P$ that are closer than $\delta\left(G^{\prime}-B\right)$ to each other and $P$ can be made longer. Suppose that we have a matching between $V\left(H^{\prime}\right)$ and $V(P)$ with endpoints $h_{1}, h_{2} \in V\left(H^{\prime}\right)$ and $v_{1}, v_{2} \in V(P)$, where $v_{1}$ is closer to $s$ on $P$ than $v_{2}$. Let $a_{1}$ denote the distance from $s$ to $v_{1}$ on $P$ and $a_{2}$ denote the distance from $v_{2}$ to $s$ on $P$. Then, if $|V(P)|+1-\left(a_{1}+a_{2}\right)<\delta\left(G^{\prime}-B\right), P$ can be enlarged using the long $\left(h_{1}, h_{2}\right)$-path of length at least $\delta\left(G^{\prime}-B\right)-2$ in $H^{\prime}$. Otherwise, $a_{1}+a_{2} \leq|V(P)|+1-\delta\left(G^{\prime}-B\right)$. In particular, $a_{1}, a_{2} \leq|V(P)|+1-\delta\left(G^{\prime}-B\right)$. Thus, $v_{1}$ is within the first $|V(P)|+2-\delta\left(G^{\prime}-B\right)$ vertices of $P$ and $v_{2}$ is within the last $|V(P)|+2-\delta\left(G^{\prime}-B\right)$ vertices of $P$.

Conclusion of Case 2. If $H^{\prime}$ is 2-connected, then either $P$ can be made longer or the following holds. The size of the maximum matching between $H^{\prime}$ and $P$ is exactly 2. Moreover, for any maximum matching between $H^{\prime}$ and $P$, the endpoint of one edge of the matching is one of the first $|V(P)|-\delta\left(G^{\prime}-B\right)+2$ vertices of $P$ and one is within the last $|V(P)|-\delta\left(G^{\prime}-B\right)+2$ vertices of $P$.

Case 3: $H^{\prime}$ is not 2-connected. Let $L$ be a leaf-block $L$ of $H^{\prime}$ and let $c$ be the cut-vertex of the leaf-block $L$. Note that $V(L) \backslash B \backslash\{c\}$ is not empty and $\delta(L-(B \cup\{c\})) \geq \delta\left(H^{\prime}-B\right)-1$.

Assume first that there is a matching of size three between $V(L)$ and $V(P)$ in $G^{\prime}$. Similar to Case 2, then there is a vertex in $V(L-(B \cup\{c\}))$ with at least $\delta\left(G^{\prime}-B\right)-\delta(L-(B \cup\{c\}))$ neighbors in $V(P)$. In this case, since the length of $P$ is at most $\delta(G-B)+k<2\left(\delta\left(G^{\prime}-B\right)-\right.$ $\delta(L-(B \cup\{c\}))-1)+2 \delta(L-(B \cup\{c\}))$, we can reroute a part of $P$ through $H^{\prime}$ and thus make it longer.

Now we may assume that the maximum matching size between $V(L)$ and $V(P)$ in $G^{\prime}$ is at most two. Again, similar to Case 2 and Claim 3 we derive that $\delta\left(L-\left(B^{\prime} \cup\{c\}\right)\right) \geq \delta\left(G^{\prime}-(B \cup\{c\})\right)-2 \geq$ $\delta\left(G^{\prime}-B\right)-3$ for some $B^{\prime} \supseteq B$. Hence, there is a path of length at least $\delta\left(G^{\prime}-B\right)-3$ between any 
pair of vertices in $L$ by Corollary 3 . It follows that there is a path of length at least $\delta\left(G^{\prime}-B\right)-3$ between an inner vertex of a leaf-block of $H^{\prime}$ and any other vertex in $H^{\prime}$.

For each leaf-block in $H^{\prime}$, there is at least one inner vertex that has at least one neighbor in $P$, otherwise $G^{\prime}$ is not 2-connected.

Suppose first that there are two inner vertices of two distinct leaf-blocks in $H^{\prime}$ that have two distinct neighbors in $V(P)$. There is always path between these two inner vertices of length at least $2\left(\delta\left(G^{\prime}-B\right)-3\right)$ : we can find two paths in each leaf-block starting in the cut vertex and ending in an inner vertex of length at least $\delta\left(G^{\prime}-B\right)-3$. Since the length of $P$ is at most $\delta(G-B)+k \leq 2\left(\delta\left(G^{\prime}-B\right)-3\right)+2$, the subpath of $P$ between their neighbours is shorter than if than the path between them through $H^{\prime}$. So we can enlarge $P$ by using this path.

Note that if there are at least two vertices $V(P)$ having at least one inner leaf-block vertex of $H^{\prime}$ as a neighbour, then we can always pick two inner vertices as described in the previous paragraph. Hence, if $P$ cannot be made longer, there is exactly one vertex $v \in V(P)$ that is connected to inner vertices of the leaf-blocks of $H^{\prime}$. Then, in fact, $\delta(L-(B \cup\{c\})) \geq \delta\left(G^{\prime}-(B \cup\{c\})\right)-1 \geq \delta\left(G^{\prime}-B\right)-2$ for each leaf-block $L$ of $H$ with cut vertex $c$. The following claim holds.

Claim 4. There is a path of length at least $\delta\left(G^{\prime}-B\right)-2$ between any inner vertex of a leaf-block and any other vertex of $H^{\prime}$.

Since $G$ is 2-connected, there is at least one other vertex $u \in V(P)$ that has neighbors in $V\left(H^{\prime}\right)$. If the distance between $u$ and $v$ on $P$ is less than $\left(\delta\left(G^{\prime}-B\right)-2\right)+2$, then $P$ can be made longer. As there is a path of length at least $\delta\left(G^{\prime}-B\right)-2$ between their neighbours in $H$. Hence, $H^{\prime}$ can only have neighbors among the first and among the last $|V(P)|+2-\delta\left(G^{\prime}-B\right)$ vertices of $P$ analogously to Case 2.

Conclusion of Case 3. If $H^{\prime}$ contains at least three vertices and is not 2-connected, then either $P$ can be made longer, or the following properties hold. All inner vertices of its leaf-blocks that have neighbors in $V(P)$ have exactly one neighbor on $P$, and this neighbour is the same for all inner vertices. This neighbour vertex is within the first (or the last) $|V(P)|+3-\delta\left(G^{\prime}-B\right)$ vertices of $P$. All other neighbours of $V\left(H^{\prime}\right)$ on $P$ are, oppositely, within the last (or the first) $|V(P)|+2-\delta\left(G^{\prime}-B\right)$ vertices of $P$.

Constructing Erdös-Gallai decomposition. We use the structural properties of $G^{\prime}$ to construct an Erdös-Gallai decomposition in graph $G^{\prime}$, and hence in $G$. We start from an $(s, t)$-path $P$ in $G^{\prime}$ and try to increase its length by applying one of the algorithms from Cases 1-3. Assume that we cannot increase the length of $P$ anymore. Then we have a path $P$ and every connected component $H^{\prime}$ of $G^{\prime}-V(P)$ should satisfy the properties summarized in the conclusion of Case 2 or Case 3. We show that in this case we either could construct in polynomial time a new path $P$ of length at least $\delta(G-B)+k$, or to construct an Erdős-Gallai decomposition.

Then each $H^{\prime}$ has neighbors within the first $k+2$ vertices of $P$ and within the last $k+2$ vertices of $P$. Denote by $P_{1}$ the shortest subpath of $P$ starting in $s$ that contains all starting neighbors (that is, neighbours that are closer to $s$ than to $t$ in $P$ ) among all possible components $H^{\prime}$. Analogously, denote by $P_{2}$ the shortest subpath of $P$ ending in $t$ that contains all ending neighbors (that is, neighbours that are closer to $t$ than to $s$ in $P$ ) among all possible $H^{\prime}$. Thus $P=P_{1} P^{\prime} P_{2}$.

Claim 5. The length of $P^{\prime}$ is at least $\delta(G-B)-k$.

Proof of Claim 5. We know that $\left|V\left(P_{1}\right)\right|,\left|V\left(P_{2}\right)\right| \leq|V(P)|-\delta\left(G^{\prime}-B\right)+2$. The length of each of $P_{1}$ and $P_{2}$ is at most $|V(P)|-\delta\left(G^{\prime}-B\right)+1$, so the length of $P^{\prime}$ is at least

$$
(|V(P)|+1)-2\left(|V(P)|-\delta\left(G^{\prime}-B\right)+1\right)=2 \delta\left(G^{\prime}-B\right)-|V(P)|>\delta(G-B)-k .
$$




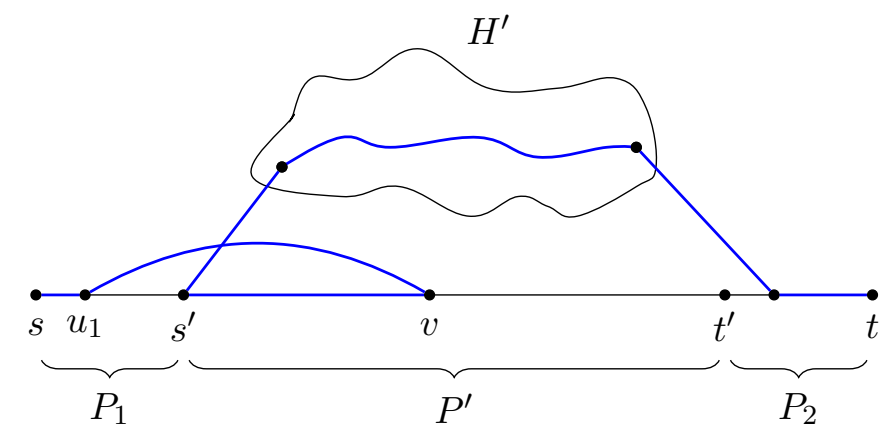

Figure 4: Construction of a long path in $G^{\prime}$ when $P^{\prime}$ is shorter than $\delta(G-B)$.

Denote by $s^{\prime}$ and $t^{\prime}$ the endpoints of $P^{\prime}$, so $P_{1}$ and $P_{2}$ are the $\left(s, s^{\prime}\right)$-subpath and the $\left(t^{\prime}, t\right)$ subpath of $P$ respectively. The following claim is rather useful.

Claim 6. There is a connected component $H^{\prime}$ in $G^{\prime}-\left(V\left(P_{1}\right) \cup V\left(P_{2}\right)\right)$ with $V\left(H^{\prime}\right) \backslash B=V\left(P^{\prime}-\right.$ $\left.\left\{s^{\prime}, t^{\prime}\right\}\right) \backslash B$.

Proof of Claim 6. We actually need to show that each vertex $v \in V\left(P^{\prime}-\left\{s^{\prime}, t^{\prime}\right\}\right)$ can only have neighbours in $V(P)$ or $B$. Suppose that there is $v \in V\left(P^{\prime}-\left\{s^{\prime}, t^{\prime}\right\}\right)$ with a neighbour $u \in V\left(G^{\prime}\right) \backslash V(P) \backslash B$. Then $u$ is in some connected component $H^{\prime \prime}$ of $G^{\prime}-V(P)$ with $\left|V\left(H^{\prime \prime}-B\right)\right| \geq$ $|\{u\}|>0$. Note that then $H^{\prime \prime}$ has a vertex with a neighbour in $P$ that is not in $V\left(P_{1}\right) \cup V\left(P_{2}\right)$. This contradicts the choice of $P_{1}$ and $P_{2}$.

We now show that the length of $P^{\prime}$ can be actually assumed to be at least $\delta(G-B)$, as agrees with the definition of Erdős-Gallai decomposition. This strengthens Claim 5 .

Claim 7. If the distance between $P_{1}$ and $P_{2}$ in $P$ is less than $\delta(G-B)$, then $G^{\prime}$ contains an $(s, t)$-path of length at least $\delta(G-B)+k$. Moreover, this path can be computed in polynomial time.

Proof of Claim \%. Suppose that the distance between $P_{1}$ and $P_{2}$ in $P$ is less than $\delta(G-B)$. Equivalently, $\left|V\left(P^{\prime}-\left\{s^{\prime}, t^{\prime}\right\}\right)\right|<\delta(G-B)-1$. Vertices in $P^{\prime}-\left\{s^{\prime}, t^{\prime}\right\}$ are adjacent in $G^{\prime}$ only to vertices in $B$ and vertices from $V(P)$ by Claim 6 . Hence, each vertex in $V\left(\left(P^{\prime}-\left\{s^{\prime}, t^{\prime}\right\}\right)-B\right)$ has at least three neighbors in $V\left(P_{1}\right) \cup V\left(P_{2}\right)$, as it has at most $\left|V\left(P^{\prime}-\left\{s^{\prime}, t^{\prime}\right\}\right)\right|-1 \leq \delta(G-B)-3$ neighbors in $V\left(P^{\prime}-\left\{s^{\prime}, t^{\prime}\right\}\right)$.

Consider the first vertex in $P^{\prime}$ that is not in $B$ and is at distance at least $\delta(G-B) / 2$ from the start of $P^{\prime}$. Denote this vertex by $v$. Note that the length of the $\left(s^{\prime}, v\right)$-subpath of $P^{\prime}$ is at most $\delta(G-B) / 2+|B|$. By Claim 5 , the distance from $v$ to the last vertex of $P^{\prime}$, i.e. the length of the $\left(v, t^{\prime}\right)$ subpath of $P^{\prime}$, is at least $\left(\left|V\left(P^{\prime}\right)\right|-1\right)-(\delta(G-B) / 2+|B|) \geq \delta(G-B)-k-\delta(G-B) / 2-|B|=$ $\delta(G-B) / 2-k-|B|$. Hence, the distance from $v$ to each of the endpoints of $P^{\prime}$ is at least $\delta(G-B) / 2-k-|B|$. Vertex $v$ has at least two neighbors in $V\left(P_{1}\right)$ or in $V\left(P_{2}\right)$, as it has at least three neighbours in $V\left(P_{1}\right) \cup V\left(P_{2}\right)$. Without loss of generality, assume that it has two neighbors in $P_{1}$.

One of its neighbors, say $u_{1}$, is different from $s^{\prime}$. Construct an $(s, t)$-path as follows. Start from $s$, move to $u_{1}$ along $P_{1}$, then from $u_{1}$ to $v$, then follow the path $P^{\prime}$ backwards from $v$ to $s^{\prime}$. By the construction of $P_{1}$, there is at least one component $H^{\prime}$ in $G^{\prime}-V(P)$ that is connected with $s^{\prime}$. Thus from $s^{\prime}$ we enter $H^{\prime}$, and follow a path of length at least $\delta\left(G^{\prime}-B\right)-2$ in $H^{\prime}$ (such path always 
exists by either Claim 3 or Claim 4 to reach some vertex in $P_{2}$. We complete the construction of the path by following along $P_{2}$ to $t$ (see Figure 4). The length of the constructed path is at least

$$
\underbrace{1}_{s \rightsquigarrow u_{1} \rightsquigarrow v}+\underbrace{\delta(G-B) / 2-k-|B|}_{v \rightsquigarrow s^{\prime}}+\underbrace{1+(\delta(G-B)-2)+1)}_{s^{\prime} \rightsquigarrow t \text { through } H^{\prime}},
$$

which equals $\frac{3}{2} \delta(G-B)-k-|B|+1>\delta(G-B)+k$.

By the claim, if the length of $P^{\prime}$ is less than $\delta(G-B)$, then we find in polynomial time the desired path and stop. Otherwise, the distance between $P_{1}$ and $P_{2}$ in $P$ is at least $\delta(G-B)$, hence $\left|V\left(P_{1}\right)\right|+\left|V\left(P_{2}\right)\right| \leq k+1$ as $|V(P)| \leq \delta(G-B)+k$.

Claim 8. The connected component $H^{\prime}$ from Claim 6 is of type (R1) in $G^{\prime}-\left(V\left(P_{1}\right) \cup V\left(P_{2}\right)\right)$, or a path of length at least $\delta(G-B)+k$ in $G^{\prime}$ can be found in polynomial time.

Proof of Claim 8. We first show that $H^{\prime}$ is 2-connected after $B$-refinements are applied to it. Denote the component $H^{\prime}$ with applied $B$-refinements by $H^{\prime \prime}$ and assume that $G^{\prime}=R_{B}\left(H^{\prime}\right)$. If $H^{\prime \prime}$ is not 2-connected, then it contains at least two leaf-blocks, as $\left|V\left(P^{\prime}-\left\{s^{\prime}, t^{\prime}\right\}\right)\right| \geq \delta(G-B)-1>2$. Since $\delta\left(H^{\prime}-B\right) \geq \delta\left(G-\left(B \cup\left(V\left(P_{1}\right) \cup V\left(P_{2}\right)\right)\right)\right) \geq \delta(G-B)-k-1$, each leaf-block of $H^{\prime \prime}$ should contain at least $\delta(G-B)-k$ vertices outside $B$. Hence, $H^{\prime}-B$ consists of at least $2(\delta(G-B)-k)-1 \geq \delta(G-B)+k \geq|V(P)|$ vertices. This is not possible since $V\left(H^{\prime}-B\right) \subseteq$ $V\left(P^{\prime}-\left\{s^{\prime}, t^{\prime}\right\}\right)$ and $\left|V\left(P^{\prime}-\left\{s^{\prime}, t^{\prime}\right\}\right)\right|<|V(P)|$.

It is left to show that the matching conditions of type (R1) are also satisfied. Assume that these conditions do not hold. Without loss of generality, assume that the maximum matching size between $V\left(H^{\prime \prime}\right)$ and $V\left(P_{1}\right)$ is at least two in $R_{B}\left(H^{\prime}\right)$. Then there are two edges $v_{1} h_{1}, v_{2} h_{2} \in E\left(G^{\prime}\right)$ with $v_{1}, v_{2} \in V\left(P_{1}\right)$ and $h_{1}, h_{2} \in V\left(H^{\prime \prime}\right)$. Without loss of generality, we assume that $v_{1}$ is closer to $s$ on $P$ than $h_{2}$. In particular, $v_{1} \neq s^{\prime}$. As $H^{\prime \prime}$ is 2-connected, then by Corollary 3 , it contains a path of length at least $\delta\left(H^{\prime \prime}-B\right)=\delta\left(H^{\prime}-B\right) \geq \delta(G-B)-k-1$ between $h_{1}$ and $h_{2}$. As discussed above in the proof of Claim 5 (see Figure 4), there is a path connecting $s^{\prime}$ with some vertex in $P_{2}$ going through a component $H$ in $G-V(P)$. Hence, there is an $\left(s^{\prime}, t\right)$-path of length at least $\delta(G-B)$ that does not have common vertices with $H^{\prime \prime}$. Then we concatenate the following paths. Take the $\left(s, v_{1}\right)$-subpath of $P_{1}$, proceed further with the edge $v_{1} h_{1}$ and the $\left(h_{1}, h_{2}\right)$-path inside $H^{\prime \prime}$, then with the edge $h_{2} v_{2}$ and the $\left(v_{2}, s^{\prime}\right)$-subpath of $P_{1}$. Finish with the $\left(s^{\prime}, t\right)$-path. The obtained path is an $(s, t)$-path of length at least

$$
\underbrace{1}_{s \rightsquigarrow h_{1}}+\underbrace{\delta(G-B)-k-1}_{h_{1} \rightsquigarrow h_{2}}+\underbrace{1}_{h_{2} \rightsquigarrow s^{\prime}}+\underbrace{\delta(G-B)}_{s^{\prime} \rightsquigarrow t},
$$

which equals to $2 \delta(G-B)-k+1>\delta(G-B)+k$. Thus, if $H^{\prime \prime}$ is not of type (R1), then we can find a long path in $G^{\prime}$ in polynomial time.

Note that every connected component in $G^{\prime}-V\left(P_{1} \cup P_{2}\right)$ corresponds either to Case 2 , or to Case 3, or to Claim 8, or is fully contained in $B$. A connected component from Case 2 or Claim 8 corresponds to (R1)-type connected components of Erdős-Gallai decompositions. The connected components from Case 3 correspond to (R2)-type and (R3)-type connected components depending on whether the vertex $v$ is from $V\left(P_{1}\right)$ or from $V\left(P_{2}\right)$. Thus, $P_{1}$ and $P_{2}$ induce an Erdős-Gallai decomposition for $P$ and $B$ in $G^{\prime}$, and hence in $G$.

The following proposition about long paths inside Erdős-Gallai components is clear from the proof of Lemma 4. 
Proposition 2. For any Erdös-Gallai component of any Erdös-Gallai decomposition in $G$ for $B \subseteq V(G)$, there is a path of length at least $\delta(G-B)-2$ between any pair of vertices of this Erdös-Gallai component.

We start to establish the properties of Erdös-Gallai components that will be exploited by the algorithm. To state the first property, we need the following definition.

Definition 3. We say that a path $P$ enters a subgraph $H$, if at least one edge of $H$ is also an edge of $P$.

Informally, the property is the following. Consider an Erdős-Gallai component $M$ for some Erdős-Gallai decomposition and consider also an $(s, t)$-path $P^{\prime}$. Path $P^{\prime}$ can hit some vertices of $M$. However, if $P^{\prime}$ enters $M$, then all vertices of $H$ hit by $P$, that is, all common vertices of $P$ and $M$, appear consecutively in $P^{\prime}$.

Lemma 5. Let $G$ be a 2-connected graph, $B \subseteq V(G), P$ be an $(s, t)$-path in $G$. Let paths $P_{1}, P_{2}$ induce an Erdös-Gallai decomposition for $P$ and $B$ in $G$. Let also $G^{\prime}$ be the graph obtained after $B$-refinements of connected components of $G-V\left(P_{1} \cup P_{2}\right)$, and let $M$ be an Erdös-Gallai component. Then for every $(s, t)$-path $P^{\prime}$ in $G^{\prime}$, if $P^{\prime}$ enters $M$, then all vertices of $M \cap V\left(P^{\prime}\right)$ appear consecutively in $P^{\prime}$.

Proof. Targeting towards a contradiction, assume that the statement of the lemma does not hold. Then there is an $(s, t)$-path $P^{\prime}$ that contains at least one edge of $M$, but vertices of $M$ does not appear consecutively in $P^{\prime}$. That is, there are vertices $v_{1}, v_{2} \in V(M), v_{1} \neq v_{2}$, such that $P^{\prime}$ is of the form $s, \ldots, v_{1}, \ldots, x, \ldots, v_{2}, \ldots, t$, where $x \notin V(M)$. No internal vertex of the $\left(s, v_{1}\right)$-subpath and the $\left(v_{2}, t\right)$-subpath of $P^{\prime}$ belongs to $V(M)$. Moreover, the $\left(v_{1}, v_{2}\right)$-subpath of $P^{\prime}$ contains at least one edge of $M$ and at least one edge outside of $M$.

Let $G^{\prime}$ be the graph obtained from $G$ after applying all possible $B$-refinements. According to the definition of Erdős-Gallai component, $M$ can be one of the following three types. Either it is a connected component of $G^{\prime}-V\left(P_{1} \cup P_{2}\right)$ (this corresponds to type (R1)), or it is a leaf-block of a connected component of $G^{\prime}-V\left(P_{1} \cup P_{2}\right)$ (this corresponds to types (R2) and (R3)]. Therefore, we consider three cases.

Case 1. Suppose that $M$ is an Erdös-Gallai component of type (R1), That is, $M$ is a connected component of $G^{\prime}-V\left(P_{1} \cup P_{2}\right)$ and also $M$ is 2-connected. Consider the $\left(s, v_{1}\right)$-subpath of $P^{\prime}$ in $G$. Since $s \in V\left(P_{1}\right)$, there exists vertex $w_{1}$ that is the last vertex on this subpath that is from $V\left(P_{1} \cup P_{2}\right)$. Then the subpath is of form $s \rightsquigarrow w_{1} \rightsquigarrow v_{1}$, where all inner vertices of the subpath $w_{1} \rightsquigarrow v_{1}$ are from $V(H) \backslash V(M)$, where $H$ is the connected component $M$ before the $B$-refinements. But after the $B$-refinement of $H$ in $G$, all inner edges of this path are contracted. Then in $G^{\prime}$ this $\left(w_{1}, v_{1}\right)$-subpath consists of just single edge $w_{1} v_{1}$.

Analogously, consider the $\left(v_{2}, t\right)$-subpath of $P^{\prime}$ and let $w_{2}$ be the first vertex from $V\left(P_{1}\right) \cup V\left(P_{2}\right)$ on this subpath. The $\left(v_{2}, w_{2}\right)$-subpath goes only through vertices in $V(H) \backslash V(M)$ in $G$ and turns into the edge between $v_{2}$ and $w_{2}$ in $G^{\prime}$.

The last subpath to consider is the $\left(v_{1}, v_{2}\right)$-subpath of $P^{\prime}$. It goes between vertices in $M$ and contains at least one edge outside $M$; hence it should contain at least one vertex in $V\left(P_{1}\right) \cup V\left(P_{2}\right)$. Let $u$ be the first vertex on this subpath that is from $V\left(P_{1}\right) \cup V\left(P_{2}\right)$. Then either the $\left(v_{1}, u\right)$-subpath or the $\left(u, v_{2}\right)$-subpath contains an edge of $M$.

First, suppose that the $\left(v_{1}, u\right)$-subpath contains an edge of $M$. Denote by $v_{3}$ the last vertex from $V(M)$ on this subpath. Then $v_{3} \neq v_{1}$ and the $\left(v_{3}, u\right)$-subpath contains only vertices in $V(H) \backslash V(M)$ as internal vertices. Hence, in this case there is an edge between $v_{3}$ and $w_{3}=u$ in $G^{\prime}$. 
Now for the case when the $\left(u, v_{2}\right)$-subpath contains an edge of $M$. Denote by $v_{3}$ the first vertex on this subpath that is from $M$. Then $v_{3} \neq v_{2}$ and the $\left(u, v_{3}\right)$-subpath does not contain vertices of $M$ as internal vertices. Denote by $w_{3}$ the last vertex in $V\left(P_{1}\right) \cup V\left(P_{2}\right)$ on this subpath. We obtain a path between $w_{3}$ and $v_{3}$ that goes only through $V(H) \backslash V(M)$ in $G$, so there is an edge between $w_{3}$ and $v_{3}$ in $G^{\prime}$.

Conclusion of Case 1. If $M$ is an Erdös-Gallai component corresponding to a connected component of type (R1), then there is a matching $v_{1} w_{1}, v_{2} w_{2}, v_{3} w_{3}$ of size three between $V(M)$ and $V\left(P_{1}\right) \cup V\left(P_{2}\right)$ in $G^{\prime}$. Hence, there is a matching between $V(M)$ and $V\left(P_{i}\right)$ of size two for some $i \in\{1,2\}$. This contradicts to the corresponding condition (R1) of Erdős-Gallai decompositions; hence Case 1 cannot occur.

Case 2. Now suppose that there is a type (R2) connected component $H$ in $G-\left(V\left(P_{1}\right) \cup V\left(P_{2}\right)\right)$ such that $M$ is a leaf-block of the component obtained after some edges of $H$ were contracted in the process of $B$-refinement $R_{B}(H)$. Denote the cut-vertex of this leaf-block $M$ by $c$. We will refer to all remaining vertices of $M$ as to inner vertices. By the definition of (R2)-type components, $N_{G^{\prime}}(V(M)) \cap V\left(P_{1}\right)=\{w\}$ for some $w \in V\left(P_{1}\right)$. Again consider the $\left(s, v_{1}\right)$-subpath, the $\left(v_{1}, v_{2}\right)$ subpath, and the $\left(v_{2}, t\right)$-subpath of $P^{\prime}$. The $\left(v_{1}, v_{2}\right)$-subpath contains a vertex $u \in V\left(P_{1}\right) \cup V\left(P_{2}\right)$ as internal vertex, so we can also break it into $\left(v_{1}, u\right)$-subpath and $\left(u, v_{2}\right)$-subpath.

Note that at least two of these four subpaths do not contain $c$. Each of these subpaths is an $(x, y)$-path for $x \in V\left(P_{1}\right) \cup V\left(P_{2}\right)$ and $y \in V(H)$. We claim that if such $(x, y)$-path does not contain $c$, then it contains $w$. Suppose that an $(x, y)$-path does not contain $c$, so $y$ is an inner vertex of $M$. This path does not contain $c$, and to reach $y$ it should reach some inner vertex of $M$ from the outside, since the path starts in $V\left(P_{1}\right) \cup V\left(P_{2}\right)$. Hence, this path should contain $w$. Otherwise there is an edge between $V\left(P_{2}\right)$ and some inner vertex of $M$ in $G^{\prime}$, which contradicts the property (R2).

Thus at least two of the four subpaths contain $w$. The only possible option for this is when $u=w$ and both $\left(v_{1}, u\right)$-subpath and $\left(u, v_{2}\right)$-subpath do not contain $c$. Then both $\left(s, v_{1}\right)$-subpath and $\left(v_{2}, t\right)$-subpath do not contain $w$, since $w$ can appear only once in $P^{\prime}$. Both of them reach an inner vertex of $M$ from the outside of $M$. If a path reaches an inner vertex of $M$ and avoids $w$, then it should contain the cut-vertex $c$. Therefore, the $\left(s, v_{1}\right)$-subpath and the $\left(v_{2}, t\right)$-subpath both contain $c$. This is contradiction, since these two paths are vertex-disjoint.

Conclusion of Case 2. If $M$ is an Erdős-Gallai component corresponding to a connected component of type (R2), then $P^{\prime}$ necessarily contains an edge between $V\left(P_{2}\right)$ and an inner vertex of $M$. This contradicts the definition of (D2)-type components.

Case 3. The case when $M$ is an Erdős-Gallai component of type (R3) is symmetrical.

In each of the three cases we obtained a contradiction with one of the properties of an ErdösGallai decomposition. This completes the proof.

In order to proceed further with the structural properties of Erdős-Gallai decompositions, we need the following definition and lemma.

Definition 4 ( $B$-leaf-block separator). Let $H$ be a connected graph that is not 2-connected and $B$ be a subset of its vertices. Let $I$ be the set of inner vertices of all leaf-blocks of $H$. We say that $S \subseteq V(H) \backslash I$ is a $B$-leaf-block separator of $H$, if $S$ separates at least one vertex in $V(H) \backslash(I \cup B)$ from $I$ in $H$.

Lemma 6. Let $H$ be a connected graph with at least one cut-vertex and let $B$ be a subset of its vertices. Let $S$ be a B-leaf-block separator of $H$. Then for any vertex $v$ that is not an inner vertex 
of a leaf-block of $H$, there is a cut-vertex $c$ of a leaf-block of $H$ and a $(c, v)$-path of length at least $\frac{1}{2}(\delta(H-B)-|S|)$ in $H$.

Proof. We assume that $\delta(H-B)>|S|$, since the other case is trivial.

Consider graph $H-(B \cup S)$. We know that there is at least one connected component in this graph that does not contain any vertex from $I$ and contains at least one vertex not in $B$. Denote this connected component by $T$. We know that $\delta(T) \geq \delta(H-(B \cup S))>1$. By Theorem 8, $T$ contains a cycle $C$ of length at least $\delta(T)+1$.

We know that $C$ is fully contained in some non-leaf-block of $H$. Denote this block by $K$. Now let $v$ be a vertex in $V(H) \backslash I$ given from the lemma statement. It is easy to see that we can always choose the vertex $c$ in a way that any $(c, v)$-path contains at least one edge of $K$. Take such vertex and an arbitrary $(c, v)$-path. Edges of $K$ induce a subpath of non-zero length in this path. Let $x, y$ be the endpoints of this subpath. We know that $x \neq y$.

We need the following claim.

Claim 9. If a 2-connected graph contains a cycle on $k$ vertices, then it contains a path of length at least $\left\lceil\frac{k}{2}\right\rceil$ between any pair of vertices.

Proof of Claim 9. Take two distinct vertices $s, t$. To show that there is a path between $s$ and $t$ of length at least $\left\lceil\frac{k}{2}\right\rceil$, we apply Menger's theorem to $\{s, t\}$ and the vertex set of the cycle of length $k$. This gives two vertex-disjoint paths going from $s$ and $t$ to two vertices $s^{\prime}$ and $t^{\prime}$ on the cycle. Take the longer arc between $s^{\prime}$ and $t^{\prime}$ on the cycle and combine it with the two paths. The resulting path is of length at least $\left\lceil\frac{k}{2}\right\rceil$.

By Claim 9, there is a path of length at least $\delta(T) / 2>\frac{1}{2}(\delta(H-B)-|S|)$ between $x$ and $y$ in $K$. Replace the subpath of the initial $(c, v)$-path with this subpath. This yields a $(c, v)$-path of desired length.

In Lemma 5, we proved that if a path enters an Erdös-Gallai component, then after leaving it, it cannot come back. The following lemma guarantees, that if we have a yes-instance, then there is a solution path that enters at least one Erdős-Gallai component.

Lemma 7. Let $G$ be a graph, $B \subseteq V(G)$ be a subset of its vertices and $P_{1}, P_{2}$ induce an ErdösGallai decomposition for an $(s, t)$-path $P$ in $G$ of length less than $\delta(G-B)+k$. Let $k$ be an integer such that $5 k+4|B|+6<\delta(G-B)$. If there exists an $(s, t)$-path of length at least $\delta(G-B)+k$ in $G$, then there exists $(s, t)$-path of length at least $\delta(G-B)+k$ in $G$ that enters an Erdös-Gallai component.

Proof. Since the length of $P$ is less than $\delta(G-B)+k$, we may assume that $\left|V\left(P_{1}\right) \cup V\left(P_{2}\right)\right|<k+2$.

Assume that there is an $(s, t)$-path $P^{\prime}$ of length at least $\delta(G-B)+k$ in $G$ that contains no edge of an Erdös-Gallai component. We show that there exists an $(s, t)$-path of length at least $\delta(G-B)+k$ that enters some Erdős-Gallai component.

The path $P^{\prime}$ path can contain only edges with endpoints in $V\left(P_{1}\right) \cup V\left(P_{2}\right) \cup B$ or edges of nonleaf-blocks of (R2) or (R3)-type components. All other edges are edges of Erdős-Gallai components.

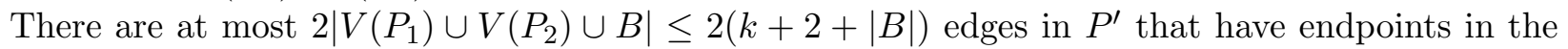
corresponding set. Hence, $P^{\prime}$ contains at least $\delta(G-B)+k-2(k+2+|B|)=\delta(G-B)-k-4-2|B|$ edges that lie inside non-leaf-blocks of separable components of the Erdös-Gallai decomposition.

Let $u$ be the vertex on $P^{\prime}$ such that the $(s, u)$-subpath of $P^{\prime}$ is of length exactly $k+1$. Denote this subpath by $P_{1}^{\prime}$. Analogously, let $v$ be the vertex on $P^{\prime}$ such that the $(v, t)$-subpath of $P^{\prime}$ is of length exactly $k$, and denote this subpath by $P_{2}^{\prime}$. Note that $P_{1}^{\prime}$ and $P_{2}^{\prime}$ are on a distance 


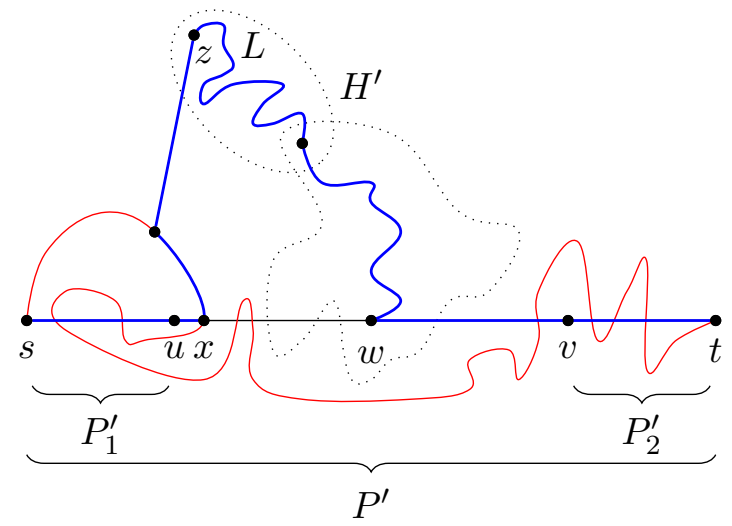

Figure 5: Constructing a path entering an Erdős-Gallai component in Case 1. The path $P$ is highlighted red. The constructed path is thick blue.

at least $\delta(G-B)-k>0$ from each other on $P^{\prime}$. The $(u, v)$-subpath of $P^{\prime}$ consists of at least $(\delta(G-B)-k-4-2|B|)-2 k>2|B|$ edges of the non-leaf-blocks. Hence, at least one non-leaf-block edge in $P^{\prime}$ is not incident to any vertex in $B$.

Let $H$ be the connected component in $G-\left(V\left(P_{1}\right) \cup V\left(P_{2}\right)\right)$ that contains this edge and let $H^{\prime}$ be its $B$-refinement. The graph $H^{\prime}$ contains at least one edge of the $(u, v)$-subpath of $P^{\prime}$, and none of these edges are incident to an inner vertex of its leaf-blocks. We note that the whole path $P^{\prime}$ cannot go through any inner leaf-block vertex of $H^{\prime}$. Suppose that this is not true and it contains such vertex. Since it does not contain any leaf-block edge, this path should enter and leave this inner vertex from the outside of $H$. And the only way to enter a (R2)-type or a (R3)-type component of the Erdös-Gallai decomposition is to go from the only vertex of $V\left(P_{1}\right)$ or of $V\left(P_{2}\right)$ correspondingly. Thus, this vertex of either $V\left(P_{1}\right)$ or $V\left(P_{2}\right)$ is contained twice on the path, and that is not possible. Consider now the graph $H^{\prime}-\left(V\left(P_{1}^{\prime}\right) \cup V\left(P_{2}^{\prime}\right)\right)$.

Case 1. Suppose that there is a connected component in this graph that contains an inner vertex of a leaf-block of $H^{\prime}$ and some vertex of the $(u, v)$-subpath of $P^{\prime}$ simultaneously. Denote this leaf-block by $L$ and the vertex of the $(u, v)$-subpath by $w$. Note that all paths between $w$ and vertices of $L$ go through the cut-vertex of $L$. If there are multiple choices of $w$ for $L$, choose the one which is the closest to the cut-vertex of $L$. As $P^{\prime}$ does not contain any inner leaf-block vertex of $H^{\prime}$, the connected component of $w$ in $H^{\prime}-\left(V\left(P_{1}^{\prime}\right) \cup V\left(P_{2}^{\prime}\right)\right)$ contains the whole leaf-block $L$. Hence, there is a path connecting $w$ with any inner vertex of $L$. Choose any inner vertex of $L$ that is connected to $V\left(P_{1}\right)$ (if $H^{\prime}$ is (R2)-type) or to $V\left(P_{2}\right)$ (if $H^{\prime}$ is (R3)-type). Denote this vertex by $z$. Since $L$ is a Erdös-Gallai component, there is a path in $L$ of length at least $\delta(G-B)-2$ connecting $z$ with the cut-vertex of $L$, so there is a $(w, z)$-path of length at least $\delta(G-B)-2$ in $H^{\prime}$. Note that the only common vertex of this path and $P^{\prime}$ is the vertex $w$. We also know that the $(s, w)$-subpath and the $(w, t)$-subpath of $P^{\prime}$ are of length at least $k+1$, since $w$ is not in $V\left(P_{1}^{\prime}\right) \cup V\left(P_{2}^{\prime}\right)$.

Now prolong the $(w, z)$-path in $G$ by going outside $H^{\prime}$ from $z$ to the vertex from $V\left(P_{1}\right)$ or $V\left(P_{2}\right)$ depending on the type of $H$, and finally go from this vertex to $t$ following the initial path $P$. We obtain a $(w, t)$-path $Q$ that has at least two common vertices with $P^{\prime}$.

Denote by $x$ the second vertex on this $(w, t)$-path that is common with $P^{\prime}$. Denote the $(w, x)$ subpath of $Q$ by $Q^{\prime}$. The path $Q^{\prime}$ is of length at least $\delta(G-B)-1$ and contains at least one Erdös-Gallai component edge, since it contains the $(w, z)$-path as a proper subpath. Suppose that $x$ is a part of the $(s, w)$-subpath of $P^{\prime}$. Then consider constructing the following path (see Figure 5). Take the $(s, x)$-subpath of $P^{\prime}$, then go following the path $Q^{\prime}$ from $x$ to $w$, and finish with the $(w, t)$ - 
subpath of $P^{\prime}$. The constructed path is of length at least $0+(\delta(G-B)-1)+(k+1) \geq \delta(G-B)+k$, so we are done. If $x$ is not a part of the $(s, w)$-subpath of $P^{\prime}$, then it is a part of the $(w, t)$-subpath of $P^{\prime}$. Then the required path is combined of the $(s, w)$-subpath of $P^{\prime}$, then of $Q^{\prime}$ and of the $(x, t)$-subpath of $P^{\prime}$. Its total length is at least $(k+1)+(\delta(G-B)-1)+0 \geq \delta(G-B)+k$.

Case 2. It is left to consider the case when $V\left(P_{1}^{\prime}\right) \cup V\left(P_{2}^{\prime}\right)$ separates all inner leaf-block vertices from all vertices of the $(u, v)$-subpath of $P^{\prime}$ that are from $V\left(H^{\prime}\right)$. Note that at least one vertex of the $(u, v)$-subpath is from $V\left(H^{\prime}\right) \backslash B$. Denote the set of all inner leaf-block vertices in $H^{\prime}$ by $I$. We know that $V\left(P_{1}^{\prime}\right) \cup V\left(P_{2}^{\prime}\right)$ separates at least one vertex in $V\left(H^{\prime}\right) \backslash B$ from $I$. Apply Lemma 6 to $H^{\prime}$ and $S=V\left(P_{1}^{\prime}\right) \cup V\left(P_{2}^{\prime}\right)$.

Suppose that $H^{\prime}$ is of type (R2), Then take any vertex in $V\left(H^{\prime}\right)$ that is connected with $V\left(P_{2}\right)$ by an edge (after the edge contractions). Denote this vertex by $y$. We know that $y$ is not in $I$, so there is a $(c, y)$-path of length at least $\frac{1}{2}\left(\delta\left(H^{\prime}-B\right)-|S|\right)$ in $H^{\prime}$ for cut-vertex $c$ of some leaf-block $L$ in $H^{\prime}$. This leaf-block has at least one inner vertex that is connected to $V\left(P_{1}\right)$ by an edge. Denote such vertex by $x$. There is a path of length at least $\delta(G-B)-3$ between $x$ and $c$ inside $L$. Combine this $(x, c)$-path with the $(c, v)$-path and obtain a path of length at least $1+(\delta(G-B)-3)+\left(\frac{1}{2}\left(\delta\left(H^{\prime}-\right.\right.\right.$ $B)-|S|))+1$ between $V\left(P_{1}\right)$ and $V\left(P_{2}\right)$. This path does not intersect internally with $P_{1}$ or $P_{2}$. Hence, there is an $(s, t)$-path in $G$ of length at least $\delta(G-B)+\frac{1}{2} \delta\left(H^{\prime}-B\right)-\frac{1}{2}|S|-1$. We know that $\delta\left(H^{\prime}-B\right) \geq \delta\left(G-\left(B \cup V\left(P_{1}\right) \cup V\left(P_{2}\right)\right)\right) \geq \delta(G-B)-k-1$. Thus, our $(s, t)$-path is of length at least $\delta(G-B)+\frac{1}{2}((\delta(G-B)-k-1)-(2(k+1))-2)=\delta(G-B)+\frac{1}{2}(\delta(G-B)-3 k-5) \geq \delta(G-B)+k$. This path contains an edge of the leaf-block $L$, which is an Erdös-Gallai component edge, so we are done.

When $H^{\prime}$ is of type (R3), the proof is symmetrical. The proof of the lemma is complete.

We are now ready to formulate a very crucial lemma of this section. It serves as a basic tool for applying recursion in Erdős-Gallai decomposition in the algorithm for LONG ERDös-GALLAI $(s, t)$ PATH. Basically, it provides a way to search for a long part of the $(s, t)$-path inside an Erdős-Gallai component wrapped up in a 2-connected subgraph of $G$.

Lemma 8. Let paths $P_{1}, P_{2}$ induce an Erdös-Gallai decomposition for an $(s, t)$-path $P$ and $B \subseteq$ $V(G)$ in graph $G$. Let $M$ be an Erdös-Gallai component in $G$. Then there is a polynomial time algorithm that outputs a 2-connected subgraph $K$ of $G$ and two vertices $s^{\prime}, t^{\prime} \in V(K)$, such that every $(s, t)$-path $P^{\prime}$ in $G$ that enters $M$, the following hold

1. $V(K) \backslash B=\left(V(M) \cup\left\{s^{\prime}, t^{\prime}\right\}\right) \backslash B$;

2. $P^{\prime}[V(K)]$ is an $\left(s^{\prime}, t^{\prime}\right)$-subpath of $P^{\prime}$ and an $\left(s^{\prime}, t^{\prime}\right)$-path in $K$;

3. $\delta\left(K-\left(B \cup\left\{s^{\prime}, t^{\prime}\right\}\right)\right) \geq \delta\left(G-\left(B \cup\left\{s^{\prime}, t^{\prime}\right\}\right)\right)$;

Proof. We consider several cases depending on the type of the connected component $H$ of $G-$ $\left(V\left(P_{1}\right) \cup V\left(P_{2}\right)\right)$ that contains $M$. We start with the simpler case, when $H^{\prime}=R_{H}(B)$ is separable. By $G^{\prime}$ we as usual denote the graph $G$ where all edges corresponding to $B$-refinements of the Erdős-Gallai decomposition induced by $P_{1}, P_{2}$ are applied.

Case 1. The component $H$ is of type (R2) (type (R3) is symmetrical as ususal). Then $M$ is some leaf-block of $H^{\prime}$. If an $(s, t)$-path $P^{\prime}$ enters $M$, then $P^{\prime}\left[V(M) \cap V\left(P^{\prime}\right)\right]$ is a path in $L$ by Lemma 5 . Moreover, we know that this path starts in an inner vertex of $M$ and ends in the cut-vertex of $M$. Denote these two vertices by $v$ and $c$ respectively.

Also, $N_{G}(H)=\{w\}$ for some $w \in V\left(P_{1}\right)$, and by definition of (R2)-type connected components, $P^{\prime}$ contains a $(w, v)$-subpath going (in either direction) internally only through vertices in $V(H) \cap B$. There are two cases of how $K$ should be constructed. 
If there is a single and the only inner vertex $v \in V(M)$ such that there is an edge between $w$ and $v$ in $G^{\prime}$, then any path $P^{\prime}$ that enters $M$ contains a path between $v$ and $c$ as a subpath. Thus, put $K:=M$ and $s^{\prime}:=v, t^{\prime}:=c$. Clearly, $K, s^{\prime}, t^{\prime}$ satisfy all three conditions in the lemma statement.

The other case is when there are at least two inner vertices in $M$ that are neighbors to $w$ in $G^{\prime}$. We cannot put $s^{\prime}$ equal to any vertex of $M$, because we cannot be sure that $P^{\prime}$ passes through a concrete inner vertex. But we are sure that $P^{\prime}$ passes through $w$. Construct $K$ in the following way. Denote by $B^{\prime}$ the set of vertices in $B$ that are reachable from $V(M) \backslash\{c\}$ in $H-\{c\}$. Then put $K:=G\left[V(M) \cup B^{\prime} \cup\{w\}\right], s^{\prime}=w, t^{\prime}=c$. Note that $K$ is an induced subgraph of $G$ and is 2-connected as $G^{\prime}[V(M) \cup\{w\}]$ is 2-connected. The first and the last two conditions in the lemma statement are satisfied, and we claim that the second one is satisfied as well.

We already know that $P^{\prime}$ contains a $(w, c)$-subpath. This subpath goes from $w$ to an inner vertex of $M$ through the vertices in $B^{\prime}$, and then follows a path inside $M$. Hence, this subpath is contained in $K$. It is left to show that no vertex from $V(M) \cup B^{\prime} \cup\{w\}$ can appear in $P^{\prime}$ outside of the $(w, c)$ subpath. We do it by contradiction. Assume that there is such vertex $v \in V(M) \cup B^{\prime} \cup\{w\}$. If $v \in V(M)$, then $v \neq c$, hence $v$ is an inner vertex of $M$. Then $P^{\prime}$ should contain a $(s, v)$-subpath or a $(v, t)$-subpath that does not go through $w$ nor $c$, but $\{w, c\}$ separates $V(M)$ from $V\left(P_{1}\right) \cup V\left(P_{2}\right)$. Thus, $v \in B^{\prime}$. Then there exists either $(s, v)$-subpath or $(v, t)$-subpath in $P^{\prime}$ that does not contain $w$ and any vertex from $V(M)$. Hence, this subpath connects $v$ with some vertex $u \in V\left(P_{2}\right)$ and goes only through $B^{\prime}$. We know that after the edge contractions for $G^{\prime}$ the vertex $v$ becomes identified with an inner vertex of $M$, so there is an edge between $u$ and this inner vertex. This is not possible by the definition of type (R2) connected components. We obtain a contradiction.

Case 2. $H$ is of type (R1), so $H^{\prime}$ is 2-connected and $M=H^{\prime}$. We know that the maximum matching size between $V\left(P_{i}\right)$ and $V(M)$ in $G^{\prime}$ is exactly one for each $i \in\{1,2\}$. For each $i$, it splits into two possible options: either $\left|N_{G^{\prime}}\left(V\left(P_{i}\right)\right) \cap V(M)\right|=1$ or $\left|N_{G^{\prime}}(V(M)) \cap V\left(P_{i}\right)\right|=\left\{w_{i}\right\}$, where $w_{i}$ has at least two neighbors in $V(M)$ in $G^{\prime}$. We now consider several subcases of Case 2 depending on the combinations of these options.

If for each $i \in\{1,2\},\left|N_{G^{\prime}}\left(V\left(P_{i}\right)\right) \cap V(M)\right|=1=\left\{v_{i}\right\}$ for some $v_{i} \in V(M)$, then, an $(s, t)$-path $P^{\prime}$ can enter or leave $M$ only through the vertices $v_{1}$ and $v_{2}$. Note that $v_{1} \neq v_{2}$, since $\left\{v_{1}, v_{2}\right\}$ separates $V(M)$ from the rest of the graph in $G$. Thus, if $P^{\prime}$ enters $M$, then it necessarily contains a $\left(v_{1}, v_{2}\right)$-subpath inside $M$. By Lemma 5, we have that $P^{\prime}\left[V\left(P^{\prime}\right) \cap V(M)\right]$ is exactly the $\left(v_{1}, v_{2}\right)$ subpath inside $M$. Thus, it is enough to put $K:=M$ and $s^{\prime}:=v_{1}, t^{\prime}:=v_{2}$. The first two and the last conditions of the lemma are satisfied for this choice of $K, s^{\prime}$ and $t^{\prime}$. Also, no vertex in $V(M) \backslash\left\{v_{1}, v_{2}\right\}$ has neighbors outside $V(H)$ in $G$, so $\left.\delta\left(K-\left(B \cup\left\{s^{\prime}, t^{\prime}\right\}\right)\right)=\delta\left(M-\left\{v_{1}, v_{2}\right\}\right]\right) \geq \delta\left(G-\left(B \cup\left\{s^{\prime}, t^{\prime}\right\}\right)\right)$, and the third condition is also satisfied.

The other case is when for each $i \in\{1,2\},\left|N_{G^{\prime}}(V(M)) \cap V\left(P_{i}\right)\right|=\left\{w_{i}\right\}$, where $w_{i}$ has at least two neighbors in $V(M)$ in $G^{\prime}$. It is easy to see that to enter or leave any vertex of $H$ in $G$, an $(s, t)$-path $P^{\prime}$ should go through $w_{1}$ and $w_{2}$. Since $G$ is 2-connected, $w_{1} \neq w_{2}$ and $P^{\prime}$ contains a $\left(w_{1}, w_{2}\right)$-subpath going internally only through vertices in $V(H)$. Put $K:=G\left[V(H) \cup\left\{w_{1}, w_{2}\right\}\right]$, $s^{\prime}:=w_{1}, t^{\prime}:=w_{2}$. Clearly, $K$ is 2-connected because $G$ is 2-connected, $\left\{w_{1}, w_{2}\right\}$ separates $V(H)$ from the rest of $G$, and degrees of $w_{1}$ and $w_{2}$ in $K$ are at least two.

We need to show that the second condition is satisfied as well. If it is not satisfied, then $P^{\prime}[V(K)]$ consists of at least two disjoint paths. We know that one of these paths is the $\left(w_{1}, w_{2}\right)$-subpath. Hence, the other one contains at least one vertex from $V(H)$ but does not contain $w_{1}$ or $w_{2}$. This is not possible since $\left\{w_{1}, w_{2}\right\}$ separates $V(H)$ from the rest of the graph.

Thus, the first two and the last condition are satisfied. It is easy to see that the third condition is satisfied as well, because vertices in $V(H)$ have no outside neighbors apart from $s^{\prime}$ and $t^{\prime}$ in $G$.

It is left to consider the case when $N_{G^{\prime}}(V(M)) \cap V\left(P_{1}\right)=\left\{w_{1}\right\}$, where $w_{1}$ has at least two 
neighbors in $V(M)$ in $G^{\prime}$, and $N_{G^{\prime}}\left(V\left(P_{2}\right)\right) \cap V(M)=\left\{v_{2}\right\}$ (the case when 1 and 2 are interchanged is symmetrical). This is the most non-clear case. We know that if $P^{\prime}$ enters $M$, then it should pass through both $w_{1}$ and $v_{2}$. Moreover, the $\left(w_{1}, v_{2}\right)$-subpath of $P^{\prime}$ goes internally only through $V(H)$. Let $B^{\prime}$ be the set of vertices reachable from $v_{2}$ by the edges in $E(H) \backslash E\left(H^{\prime}\right)$.

The difficulty beyond choosing $K$ in this case is to satisfy the second condition. We split on two cases.

Assume that there is no edge between $w_{1}$ and $v_{2}$ in $G^{\prime}$. Then put $K:=G\left[\left(V(H) \cup\left\{w_{1}\right\}\right) \backslash B^{\prime}\right]$, $s^{\prime}:=w_{1}$ and $t^{\prime}:=v_{2}$. Clearly, $K$ is equal to $G\left[V(H) \cup\left\{w_{1}\right\}\right]$ with applied $B$-refinements so it is 2-connected. The first, the third and the fourth condition of the lemma are satisfied by the arguments similar to the cases considered above. It is left to show that the second condition is satisfied. Suppose that $P^{\prime}[V(K)]$ contains a path different from the $\left(w_{1}, v_{2}\right)$-subpath. Then there is at least one vertex $u \in V(K) \backslash V(M)$ that is not on this subpath. Then $P^{\prime}$ should contain either an $(s, u)$-subpath or an $(t, u)$-subpath that does not go through $w_{1}$ or $v_{2}$. Moreover, this subpath does not contain any vertex of $V(M)$ by Lemma 5. Denote by $x$ the last vertex on this supbath that is not from $V(K)$. Then $P^{\prime}$ contains an $(x, u)$-subpath, where $x \in V\left(P_{1}\right) \cup V\left(P_{2}\right)$ After the $B$-refinement of $H$, this path yields an edge in $G^{\prime}$ between $x$ and $y$ for some $y \in V(M)$. This is only possible when either $x=w_{1}$ or $y=v_{2}$.

The case $x=w_{1}$ is not possible because the $(x, u)$-subpath does not contain $w_{1}$. Hence, it should be the case that $y=v_{2}$. Then $u$ is a vertex reachable from $v_{2}$ in $H$ outside $V(M)$. That is, $u \in B^{\prime}$. Hence, $u \notin V(K)$. We obtain a contradiction, so all four conditions are satisfied.

It is left to consider the case when there is an edge between $w_{1}$ and $v_{2}$ in $G^{\prime}$. It is clear that in this case the graph $G\left[V(H) \cup\left\{w_{1}\right\}\right]$ is 2-connected. Unfortunately, we cannot put $K$ equal to this graph because this might break the second condition of the lemma.

We already know, however, that the graph $K:=G\left[\left(V(H) \cup\left\{w_{1}\right\}\right) \backslash B^{\prime}\right]$ with $s^{\prime}:=w_{1}$ and $t^{\prime}:=v_{2}$ would satisfy all conditions of the lemma except, possibly the first. Thus, there are two cases.

When the graph $G\left[\left(V(H) \cup\left\{w_{1}\right\}\right) \backslash B^{\prime}\right]$ is 2-connected, then consider $K, s^{\prime}$ and $t^{\prime}$ similarly to the case when there is no edge between $w_{1}$ and $v_{2}$.

Otherwise, the graph $G\left[\left(V(H) \cup\left\{w_{1}\right\}\right) \backslash B^{\prime}\right]$ is not 2-connected. Then $w_{1}$ is connected to exactly two vertices from $V(M)$ in $G^{\prime}$. One of these two vertices is $v_{2}$. The other one we denote by $v_{1}$. Then $P^{\prime}$ necessarily contains a $\left(v_{1}, v_{2}\right)$-subpath inside $M$. Then it is sufficient to put $K:=M$, $s^{\prime}:=v_{1}, t^{\prime}:=v_{2}$, as $M$ is 2-connected.

The proof is complete.

\subsection{Algorithm for Long Erdős-Gallai $(s, t)$-Path}

We are almost set to proceed with the proof of Theorem 5. The algorithm is based on Lemmata 4, 5, 6, and 8 on properties of Erdös-Gallai decompositions. For the proof of the correctness of the algorithm, we will need one more lemma.

Lemma 9. Let $G$ be a 2-connected graph with $B \subseteq V(G)$ such that $\frac{6}{5} \delta(G-B) \geq|V(G)|$ and $\delta(G-B) \geq 4|B|$. Then for any pair of distinct vertices $s, t \in V(G)$, the longest $(s, t)$-path in $G$ contains all vertices from $V(G-B)$.

Proof. The proof is by contradiction. Suppose that there is an $(s, t)$-path $P$ in $G$ such that the length of $P$ is maximum possible, but there is $v \in V(G) \backslash B$ with $v \notin V(P)$. By Corollary 3 , the length of $P$ is at least $\delta(G-B)$. Hence, $|V(P-B)|>\delta(G-B)-|B|$. Then $v$ has at most $|V(G-B)|-|V(P-B)|<\frac{1}{5} \delta(G-B)+|B|$ neighbors outside $V(P)$. Hence, $v$ has more than $\frac{4}{5} \delta(G-B)-|B|$ neighbors from $V(P)$. 
Note that $v$ should not have any two consecutive vertices in $P$ as neighbors, otherwise $P$ can be made longer. Hence, $2\left(\frac{4}{5} \delta(G-B)-|B|\right)<|V(P)|$. Equivalently, $|V(P)|>|V(G)|+\frac{3}{5} \delta(G-$ $B)-2|B| \geq|V(G)|$. This is a contradiction.

For reader's convenience, we restate Theorem 5 here.

Theorem 5. LONG ERdős-Gallai $(s, t)$-PATH is solvable in $2^{\mathcal{O}(k+|B|)} \cdot n^{\mathcal{O}(1)}$ running time on 2-connected graphs.

Proof. The recursive algorithm is presented in Algorithm 2. Note that this algorithm requires that $s, t \in B$ in the given input instance. Any instance can be reduced to instance with this restriction by adding $s, t$ into $B$ and increasing $k$ by at most two. This changes the parameters by a constant value and does not significantly affect the running time of the algorithm. Also, this algorithm does not just determine whether the given instance is a yes-instance. If the given instance is a no-instance, the algorithm also outputs the maximum length of an $(s, t)$-path in $G$ in the form $\delta(G-B)+x$, where $x \geq 0$ and $x<k$. Note that algorithm actually also finds a path of such length, and it possible to change it so the path is in the output of the algorithm. We now go through the lines of the algorithm to explain its correctness.

The first two conditional operators handle the most trivial cases of the problem. The first conditional operator is for the case $k=0$, which corresponds to trivial yes-instances by Corollary 3 . The second operator ensures that parameters $k$ and $|B|$ are small enough compared to $\delta(G-B)$ to apply results discussed earlier in this section. If they are not, the algorithm just employs the algorithm from Theorem 12 for Long $(s, t)$-PATH, which works in $2^{\mathcal{O}(\delta(G-B)+k)} \cdot n^{\mathcal{O}(1)}=$ $2^{\mathcal{O}(k+|B|)} \cdot n^{\mathcal{O}(1)}$.

When the third conditional operator is reached, Lemma 4 can indeed be applied to the input instance. Thus, in polynomial time either an $(s, t)$-path of length at least $\delta(G-B)+k$ is found, or an $(s, t)$-path $P$ with $V(P) \cup B=V(G)$ is found, or an $(s, t)$-path $P$ and two paths $P_{1}, P_{2}$ are found. The paths $P_{1}$ and $P_{2}$ induce an Erdös-Gallai decomposition for $P$ in $(G, B)$. If the path of length at least $\delta(G-B)+k$ is found, our algorithm correctly decides that the given instance is a yes-instance and stops. Otherwise, it enters the third conditional operator body.

The conditional operator in line 24 checks that we should deal with the case covered by Lemma 9 We shall now explain this in detail. Suppose that we enter the conditional operator body, i.e., $V(P) \cup B=V(G)$. Since the length of $P$ is at most $\delta(G-B)+k-1$, we get that $\delta(G-B)+k+|B| \geq$ $|V(G)|$. Since this operator can be reached only if $5(k+|B|) \leq|V(G)|$, we can apply Lemma 9 to our instance. We can now look for an $(s, t)$-path that contains all vertices in $V(G-B)$. Clearly, any such path is a hamiltonian path in the graph $G-B^{\prime}$ for some $B^{\prime} \subseteq B$ with $s, t \notin B^{\prime}$. To achieve that any hamiltonian path in $G-B^{\prime}$ corresponds to an $(s, t)$-path, we add two additional vertices $s^{\prime}, t^{\prime}$ of degree one to obtain the graph $H$.

Moreover, in the graph $H$ all vertices have degree at least $\delta(G-B)$, except, probably, at most $|B|+2$ vertices. We know that $2 \delta(G-B)>|V(H)|$, so we can apply one of the two FPT-algorithms from Theorem 13 for solving Hamiltonian PATH in $H$. This algorithm runs in $2^{\mathcal{O}(|B|+2)} \cdot n^{\mathcal{O}(1)}$ time. Thus, the longest path in $G-B$ is found by the algorithm for the correct choice of $B^{\prime}$.

We now move to the most crucial part of the algorithm. This part deals with Erdös-Gallai components of the Erdös-Gallai decomposition induced by $P_{1}$ and $P_{2}$. We note that when line 13 of the algorithm is reached, there are at least two distinct Erdős-Gallai components of the ErdősGallai decomposition of $(G, B)$ by definition. By Lemma 7 and Lemma 8, if the given instance is a yes-instance, there is an Erdös-Gallai component that contains a long subpath of the desired path. Let $M$ be an Erdös-Gallai component fixed by the foreach cycle. Lemma 8 applied to this Erdős-Gallai component yields a triple $K, s^{\prime}, t^{\prime}$. The following lines of the algorithm focus on finding 


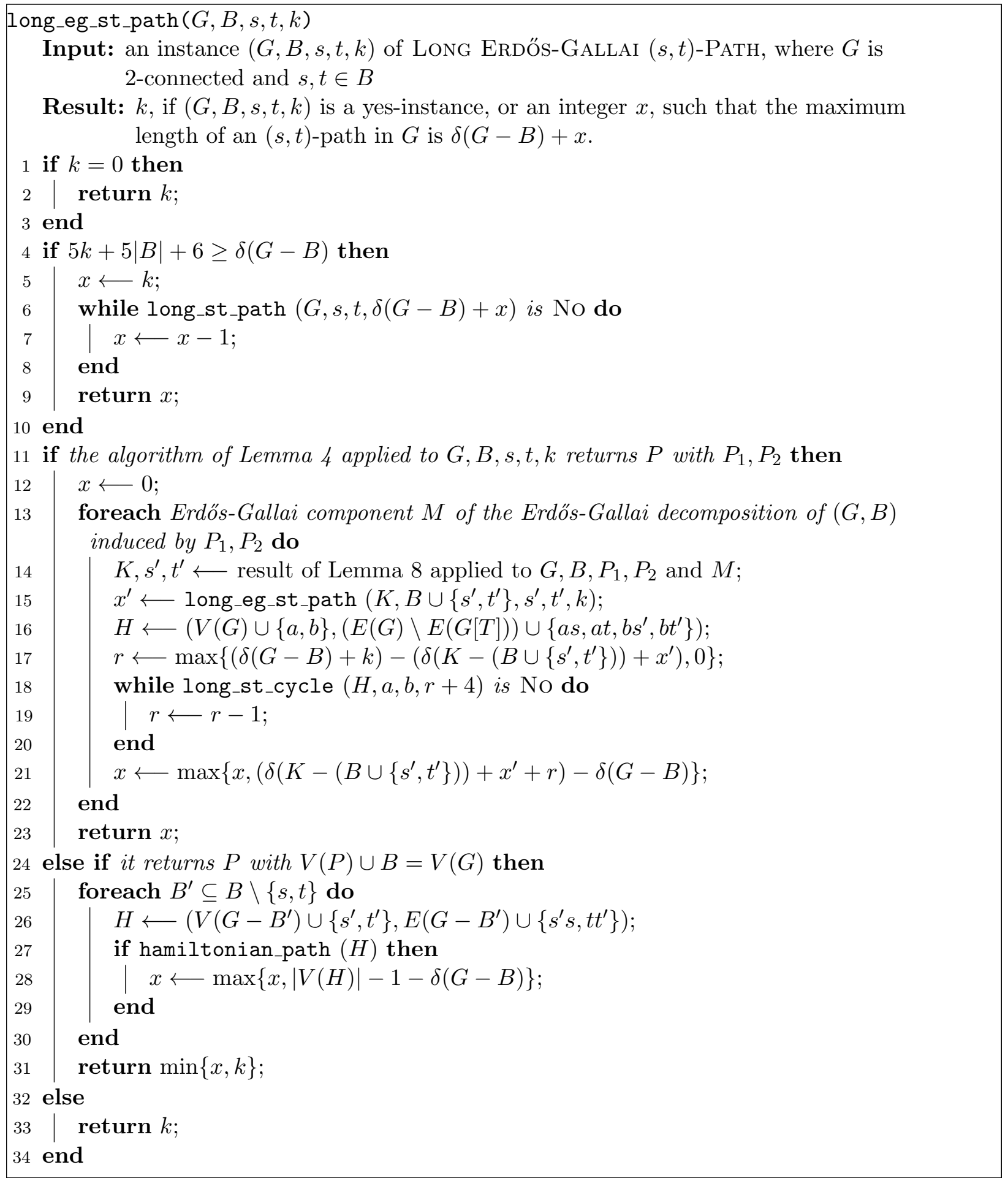

Algorithm 2: Recursive algorithm solving LONG ERDŐS-GAllai $(s, t)$-PATH on 2connected graphs.

maximum $x^{\prime}$ such that there is an $\left(s^{\prime}, t^{\prime}\right)$-path in $K$ of length at least $\delta\left(K-\left(B \cup\left\{s^{\prime}, t^{\prime}\right\}\right)\right)+x^{\prime}$. We know that such path in $K$ exists for $x^{\prime}=0$ by Corollary 3 . We shall analyze the running time of this recursion later in this proof. 
Note that any $\left(s^{\prime}, t^{\prime}\right)$-path in $K$ can be expanded to an $(s, t)$-path in $G$ using at least $p:=$ $\left|\left\{s^{\prime}, t^{\prime}\right\} \backslash\{s, t\}\right|$ edges. Also, $s, t \in B$, so $\left|B \cup\left\{s^{\prime}, t^{\prime}\right\}\right| \leq|B|+p$. Hence, if there is an $\left(s^{\prime}, t^{\prime}\right)$-path in $K$ of length at least $\delta\left(K-\left(B \cup\left\{s^{\prime}, t^{\prime}\right\}\right)\right)+x^{\prime} \geq \delta\left(G-\left(B \cup\left\{s^{\prime}, t^{\prime}\right\}\right)\right)+x^{\prime} \geq \delta(G-B)-p+x^{\prime}$, there is a path of length at least $\delta(G-B)+x^{\prime}$ in $G$. It follows that if $x^{\prime} \geq k$ then the algorithm can safely decide that the given instance is a yes-instance.

Otherwise, the maximum possible $x^{\prime}<k$ is found and it is left for the algorithm to expand the $\left(s^{\prime}, t^{\prime}\right)$-path in $K$ to an $(s, t)$-path in $G$. That is, it needs to find two vertex-disjoint paths of sufficient total length going from $s^{\prime}, t^{\prime}$ to $s, t$ in $G$. An additional restriction for these paths is that they should not contain any edge of $M$. Since the sufficient total length is bounded by $k+2$, we can safely employ the algorithm for LONG $(s, t)$-CyCLE, Theorem 4 from Section 5 , running in $2^{\mathcal{O}(k)} \cdot n^{\mathcal{O}(1)}$ time.

The correctness of the algorithm is now clear and we move to analyze the recursion running time. We know that without the recursive call, the algorithm runs in $2^{\mathcal{O}(k+|B|)} \cdot n^{\mathcal{O}(1)}$ time. For convenience, we write this running time bound in the form $2^{\mathcal{O}(k+|B|)} \cdot(n-2)^{\mathcal{O}(1)}$. Note that this is possible since $n>2$ for any 2 -connected graph $G$. Thus, we can already assume that if the algorithm runs without making recursive calls, it runs in $2^{c_{1}(k+|B|)} \cdot(n-2)^{c_{2}}$ time, where $c_{1}, c_{2} \geq 1$ are constant integers given by the non-recursive subroutine.

Since the recursive call is made when the graph contains at least two Erdös-Gallai components, it is always made to an instance with the smaller number of vertices. We will now prove that our algorithm runs in $2^{c_{1}(k+|B|)} \cdot(n-2)^{c_{2}+1}$ time by induction on $n$.

The base of our induction are instances for which no recursive calls are made. Consider an instance for which at least two recursive calls are made. We want to prove that the algorithm running time $2^{\mathcal{O}(k+|B|)} \cdot n^{\mathcal{O}(1)}$. First note that the parameter $k+|B|$ does not increase in a recursive call, because $\left|\left(B \cup\left\{s^{\prime}, t^{\prime}\right\}\right) \cap V(K)\right| \leq|B \cap V(G)|$.

Let $q \geq 2$ be the number of Erdôs-Gallai components in $G$. For $i \in[q]$, denote by $K_{i}, s_{i}^{\prime}, t_{i}^{\prime}$ the triple given by Lemma 8 for the $i$-th Erdős-Gallai component of $G$. Denote also $n_{i}:=\left|V\left(K_{i}\right)\right|$. The running time of the algorithm for the instance given by $K_{i}$ is at most $2^{c_{1}(k+|B|)} \cdot\left(n_{i}-2\right)^{c_{2}+1}$ by induction. Note that all $q$ sets $V\left(K_{i}\right) \backslash\left\{s_{i}^{\prime}, t_{i}^{\prime}\right\}$ are pairwise disjoint. Also, none of these sets contains $s$ or $t$. Hence, $\sum_{i=1}^{q}\left(n_{i}-2\right) \leq n-2$. We now want to upper-bound the sum $\sum_{i=1}^{q}\left(n_{i}-2\right)^{c_{2}+1}$.

Proposition 3. Let $a_{1}, a_{2}, \ldots, a_{q}$ be a sequence of $q \geq 2$ positive integers with $\sum_{i=1}^{q} a_{i}=n$. Let $x>1$ be an integer. Then $\sum_{i=1}^{q} a_{i}^{x} \leq(n-1)^{x}+1 \leq n^{x}-n^{x-1}$.

Proof. First, we show that the maximum of the sum $\sum_{i=1}^{q} a_{i}^{x}$ is achieved with $q=2, a_{1}=n-1$, $a_{2}=1$, if the sum $\sum_{i=1}^{q} a_{i}=n$ is fixed. To show that the maximum cannot be achieved with $q>2$, it is enough to see that replacing $a_{q-1}$ and $a_{q}$ with $a_{q-1}+a_{q}$ yields a greater total sum, as $\left(a_{q-1}+a_{q}\right)^{x}>a_{q-1}^{x}+a_{q}^{x}$.

We know that the maximum is achieved with $a_{1}^{x}+a_{2}^{x}$ for some positive integers $a_{1}, a_{2}$ with $a_{1}+a_{2}=n$. Without loss of generality, we can assume that $a_{1} \geq a_{2}$. Suppose that $a_{2}>1$. Consider replacing $a_{1}$ with $a_{1}+1$ and $a_{2}$ with $a_{2}-1$. We need to show that the total sum does not decrease, i.e., $\left(a_{1}+1\right)^{x}+\left(a_{2}-1\right)^{x} \geq a_{1}^{x}+a_{2}^{x}$, or $\left(a_{1}+1\right)^{x}-a_{1}^{x} \geq a_{2}^{x}-\left(a_{2}-1\right)^{x}$. Rewrite the left and the right part to obtain

$$
\sum_{i=0}^{x}\left(\begin{array}{l}
x \\
i
\end{array}\right) a_{1}^{i}-a_{1}^{x} \geq a_{2}^{x}-\sum_{i=0}^{x}\left(\begin{array}{l}
x \\
i
\end{array}\right) a_{2}^{i}(-1)^{x-i} .
$$

Then

$$
\sum_{i=0}^{x-1}\left(\begin{array}{l}
x \\
i
\end{array}\right) a_{1}^{i} \geq-\sum_{i=0}^{x-1}\left(\begin{array}{l}
x \\
i
\end{array}\right) a_{2}^{i}(-1)^{x-i}
$$


and

$$
\sum_{i=0}^{x-1}\left(\begin{array}{l}
x \\
i
\end{array}\right)\left(a_{1}^{i}+a_{2}^{i}(-1)^{x-i}\right) \geq 0
$$

Each summand of the sum in the last inequality is non-negative since $a_{1}^{i} \geq a_{2}^{i}$ for any $i \geq 0$. Thus, the initial inequality holds and we can replace $\left(a_{1}, a_{2}\right)$ with $\left(a_{1}+1, a_{2}-1\right)$ if $a_{2}>1$ so the total sum does not decrease. Hence, the maximum is achieved with $a_{1}=n-1$ and $a_{2}=1$.

It is left to show that $(n-1)^{x}+1 \leq n^{x}-n^{x-1}$. We rewrite it as

$$
1 \leq(n-1) \cdot\left(n^{x-1}-(n-1)^{x-1}\right),
$$

which holds as $n>1$ and $x>1$. The proof is complete.

With this proposition, we have that the running time of the algorithm is upper-bounded by

$$
2^{c_{1}(k+|B|)} \cdot(n-2)^{c_{2}}+2^{c_{1}(k+|B|)} \cdot \sum_{i=1}^{q} n_{i}^{c_{2}+1} \leq 2^{c_{1}(k+|B|)} \cdot\left((n-2)^{c_{2}}+\left((n-2)^{c_{2}+1}-(n-2)^{c_{2}}\right)\right),
$$

so the induction hypothesis holds. This concludes the proof.

\section{$7 \quad$ Algorithm for small vertex covers}

In this section we prove Theorem 6 stating that Long DiRAC CyCle / VerTex Cover Above DEGREE is solvable in $2^{\mathcal{O}(p+|B|)} \cdot n^{\mathcal{O}(1)}$ running time. Recall that the task of this problem is, given a graph $G$, a subset of vertices $B$, a vertex cover $S$ of $G$ of size $\delta(G-B)+p$ and a nonnegative integer $k$, decide whether $G$ has a cycle of length at least $2 \delta(G-B)+k$. We start by assembling combinatorial results about paths and vertex covers, which we later use in the algorithm.

The following lemma provides conditions when a part of long cycle $C$ can be rerouted through any sufficiently large independent set.

Lemma 10. Let $G$ be a graph with a given subset of vertices $B$ and a vertex cover $S$ such that $S \supseteq B$ and $|S|=\delta(G-B)+p$ for some $p \geq 0$. Let $k$ be a non-negative integer and let $X \subseteq I=V(G) \backslash S$ be such that $|X|=\delta(G-B)-3 p$. If $G$ has a cycle $C$ of length $2 \delta(G-B)+k$, then it also has a cycle $C^{\prime}$ such that

- The length of $C^{\prime}$ is $2 \delta(G-B)+k$,

- $C^{\prime}$ contains all vertices of $X$, and

- $V(C) \cap S=V\left(C^{\prime}\right) \cap S$.

Proof. Because $S$ is a vertex cover, the length of any cycle in $G$ does not exceed $2|S|$. Hence if $G$ contains a cycle of length $2 \delta(G-B)+k$, we have that $k \leq 2 p$.

Suppose that $G$ contains a cycle $C$ of length $2 \delta(G-B)+k$. Among all cycles of length $2 \delta(G-B)+k$, we select a cycle $C^{\prime}$ such that $V(C) \cap S=V\left(C^{\prime}\right) \cap S$ and, subject to that, with the maximum number of vertices from $X$. We claim that all vertices of $X$ are in $C^{\prime}$. Targeting towards a contradiction, assume that there is a vertex $x \in X$ that is not in $C^{\prime}$. Let $S^{\prime}=S \cap V\left(C^{\prime}\right)$. Note that $\left|S^{\prime}\right| \geq \frac{\left|C^{\prime}\right|}{2}=\delta(G-B)+\frac{k}{2}$. Because $S$ is a vertex cover, all neighbors of $x$ are in $S$. Then $x$ has at least $\delta(G-B)$ neighbors in $S$ and, therefore, all but $p$ vertices of $S^{\prime}$ are adjacent to $x$. 
Let $v_{1}, v_{2}, \ldots, v_{\left|S^{\prime}\right|}$ be the vertices of $S^{\prime}$ in the order they appear on the cycle $C^{\prime}$. Note that for each $i \in\left\{1, \ldots,\left|S^{\prime}\right|\right\}$, vertices $v_{i}$ and $v_{i+1}$ (and $\left.v_{\left|S^{\prime}\right|}, v_{1}\right)$ are either adjacent vertices in $C^{\prime}$, or there exists exactly one vertex from $I$ that is between them in $C^{\prime}$. We want to show that there exists at least one pair $\left\{v_{i}, v_{i+1}\right\}$ such that both $v_{i}$ and $v_{i+1}$ are adjacent to $x$ and a vertex $u \in I \backslash X$ is between $v_{i}$ and $v_{i+1}$ in $C^{\prime}$. If such a pair exists, then by swapping $u$ and $x$ in $C^{\prime}$, we would obtain a cycle that has a larger number of vertices from $X$ leading to a contradiction.

There are at least $\delta(G-B)+k-p$ vertices from $I$ in $C^{\prime}$, so there are at least $\delta(G-B)+k-p$ pairs $\left\{v_{i}, v_{i+1}\right\}$ that have a vertex from $I$ between them. We know that at most $p$ vertices in $S^{\prime}$ are not adjacent to $x$. Since each vertex in $S^{\prime}$ is a member of at most two pairs, vertex $x$ is adjacent to all but $2 p$ such pairs $\left\{v_{i}, v_{i+1}\right\}$.

Suppose that $C^{\prime}$ already contains $t \geq 0$ vertices from $X$. Note that by our assumption, $t<$ $|X|=\delta(G-B)-3 p$, thus $\delta(G-B)+k-p-2 p-t>0$. Therefore, at least one pair of vertices $\left\{v_{i}, v_{i+1}\right\}$ is adjacent to $x$ and $v_{i} u v_{i+1}$ is a subpath of $C^{\prime}$ for some $u \in I \backslash X$. Therefore, by rerouting $C^{\prime}$ through $x$, instead of $u$, we construct a cycle $C^{\prime \prime}$ of length $2 \delta(G-B)+k$, such that $V(C) \cap S=V\left(C^{\prime \prime}\right) \cap S$, and $C^{\prime \prime}$ containing $t+1$ vertices of $X$. But by our assumption, cycle $C^{\prime}$ contains the maximum number of vertices $t$ from $X$. We achieved the contradiction that concludes the proof of the lemma.

We will need the following two simple facts about the number of vertices in a vertex cover that have a small amount of neighbors outside the vertex cover.

Lemma 11. Let $G$ be a graph, $S \subseteq V(G)$ be a vertex cover of $G$, and let $I=V(G) \backslash S \neq \emptyset$. Let $d=\min _{v \in I} \operatorname{deg}_{G}(v), b=|S|-d \geq 0$, and $\beta=\frac{d}{|I|}$. Then for any $\alpha \in\left(0, \frac{1}{\beta}\right)$, the number of vertices in $S$ having less that $\alpha d$ neighbors in $I$ is strictly less than $\frac{b}{1-\alpha \beta}$.

Proof. Let $s$ be the number of vertices in $S$ with less than $\alpha d$ neighbors in $I$. On one hand, the number of edges between $I$ and $S$ is at least $d|I|$. On the other hand, it is less than $\alpha d s+(|S|-s)|I|=$ $\alpha d s+(d+b-s)|I|$. Hence,

$$
d|I|<\alpha d s+(d+b-s)|I|
$$

This is equivalent to

$$
d<\frac{\alpha d s}{|I|}+d+b-s
$$

Thus

$$
b>s \cdot\left(1-\alpha \cdot \frac{d}{|I|}\right)=s \cdot(1-\alpha \beta),
$$

and we conclude that $s<\frac{b}{1-\alpha \beta}$.

Lemma 12. Let $G$ be a graph with $B \subseteq V(G)$ and a vertex cover $S \supseteq B$ with $|S|=\delta(G-B)+p$, where $0<p<\delta(G-B) / 8$. Then for any $X \subseteq V(G) \backslash S$ with $|X| \geq \delta(G-B)-3 p$, at most $2 p$ vertices in $S$ have less than $2 p$ neighbors in $X$.

Proof. Consider the graph $G[S \cup X]$. Apply Lemma 11 to this graph with $I=X$. Clearly, $d \geq \delta(G-B)>8 p$, because $B \subseteq S$ and $p<\delta(G-B) / 8$. Therefore, $b \leq p$. Because $p<\delta(G-B) / 8$, we also have that $\beta=d /|X| \leq|S| /(\delta(G-B)-3 p)=(\delta(G-B)+p) /(\delta(G-B)-3 p) \leq$ $1+4 p /(\delta(G-B)-3 p)<\frac{9}{5}$. Pick $\alpha=\frac{1}{4}$, so $\alpha \beta<\frac{9}{20}$. By Lemma 11 , at most $p /(1-\alpha \beta)<\frac{20}{11} p<2 p$ vertices in $S$ have less than $\alpha d>\frac{1}{4} \cdot 8 p=2 p$ neighbors in $I=X$. 
The following structural lemma provides necessary and sufficient conditions for the existence of a long cycle in graph crossing specified subsets of the vertex cover and the independent set. These conditions can be checked in FPT time (Lemma 14), and both lemmata are the crucial components in the proof of Theorem 6 .

Lemma 13. Let $G$ be a graph, $B \subseteq V(G)$, and let $p>0$ be an integer such that $p<\delta(G-B) / 8$. Assume that $G$ has a vertex cover $S$ such that $|S|=\delta(G-B)+p$ and $B \subseteq S$. Let $k \geq 0$ be an integer and let $X \subseteq I=V(G) \backslash S$ such that $|X| \geq \delta(G-B)-3 p$. Let $A \subseteq S$ be the set of vertices of $S$ with at least $p+1$ neighbors in $X$, and let $Z=S \backslash A$. Then there is a cycle of length $2 \delta(G-B)+k$ in $G$ containing all vertices in $X \cup Z$ if and only if there is a set $Y \subseteq I$ and a path cover $\mathcal{P}$ of $G[S \cup Y]$, such that:

(i) $\mathcal{P}$ consists of $|S|+k-2 q-|Y|$ paths, where $\frac{k}{2} \leq q \leq p$,

(ii) $\mathcal{P}$ contains no path with an endpoint in $Z$ or $Y$,

(iii) At least $p-q$ of paths in $\mathcal{P}$ are paths of length 0 , that is, covering a single vertex of $A$,

(iv) $|Y| \leq 2|Z|$,

(v) $|X \cup Y| \leq \delta(G-B)+k-q$, and

(vi) $|I| \geq \delta(G-B)+k-q$.

Proof. Let $C$ be a cycle of length $2 \delta(G-B)+k$ in $G$ containing all vertices from $X \cup Z$. Define $Y \subseteq I$ to be the set of the vertices of $C$ in $I$ having neighbors in $Z$ in the cycle. Clearly, $|Y| \leq 2|Z|$ satisfying (iv). Let $S^{\prime}=S \cap V(C)$ and define $q:=\left|S^{\prime}\right|-\delta(G-B)$. Note that $q \geq \frac{k}{2}$ because $2\left|S^{\prime}\right| \geq|C|$, and that $q \leq p$ because $\left|S^{\prime}\right| \leq|S|$. Hence, the conditions for $q$ in (i) are satisfied. Since $X \cup Y \subseteq V(C)$ and $|V(C) \backslash(X \cup Y)| \geq\left|S^{\prime}\right|=\delta(G-B)+q$, we have that $|X \cup Y| \leq \delta(G-B)+k-q$ and (v) holds. Notice that $|I| \geq|V(C) \cap I|=|V(C)|-\left|S^{\prime}\right|=(2 \delta(G-B)+k)-(q+\delta(G-B))=\delta(G-B)+k-q$ and (vi) is fulfilled.

Because $p<\delta(G-B) / 8$ and $|X| \geq \delta(G-B)-3 p,|Z|<2 p$ by Lemma 12 . Then $|Y| \leq 2|Z|<4 p$ and, therefore, $\left|S^{\prime}\right|+|Y|<\delta(G-B)+q+4 p \leq \delta(G-B)+5 p$. Since $C$ has $2 \delta(G-B)+k \geq 2 \delta(G-B)$ vertices and $\delta(G-B) \geq 8 p$, we obtain that $\left|S^{\prime}\right|+|Y|<|C|$. This means that $C\left[S^{\prime} \cup Y\right]$ is a proper subgraph of $C$, that is, the union of disjoint paths. Consider the path cover $\mathcal{P}^{\prime}$ of $S^{\prime} \cup Y$ which is produced by $C$, that is, $\mathcal{P}^{\prime}$ is the set of paths that are connected components of $C\left[S^{\prime} \cup Y\right]$ (see Figure 6). It consists of $|C|-\left|S^{\prime}\right|-|Y|$ paths, as each vertex from $V(C) \backslash\left(S^{\prime} \cup Y\right)$ on $C$ is a neighbor to exactly two endpoints in the path cover produced by $C$. Note also that the endpoints of each path of $\mathcal{P}^{\prime}$ are in $A$. This path cover still does not cover vertices in $S \backslash S^{\prime}$, so we add $|S|-\left|S^{\prime}\right|=p-q$ paths of zero length covering each vertex from $S \backslash S^{\prime} \subseteq A$; this satisfies (iii). The obtained path cover $\mathcal{P}$ is a path cover of $S \cup Y$ consisting of exactly $|C|+|S|-2\left|S^{\prime}\right|-|Y|=|S|+k-2 q-|Y|$ paths implying (i). Since all the path in $\mathcal{P}^{\prime}$ have their endpoints in $A$ and each or $p-q$ trivial paths is a vertex of $A$, we obtain that (ii) is fulfilled. We conclude that $\mathcal{P}$ satisfies conditions (i) - (vi) in the statement of the lemma.

We now prove the opposite direction. Let $Y \subseteq I$ and let $\mathcal{P}$ be a path cover of $G[S \cup Y]$ satisfying conditions (i)-(vi) of the lemma. In particular, $|\mathcal{P}|=|S|+k-2 q-|Y|$, where $\frac{k}{2} \leq q \leq p$, by (i). We show that there exists a cycle of length $2 \delta(G-B)+k$ containing all vertices of $X \cup Z$. We remove from $\mathcal{P}$ arbitrary $p-q$ zero length paths covering single vertices of $A$ using (iii). The obtained set of paths $\mathcal{P}^{\prime}$ consists of $|S|-p-q+k-|Y|=\delta(G-B)+k-q-|Y|$ paths and covers $G\left[S^{\prime} \cup Y\right]$, where $S^{\prime} \subseteq S$ and $\left|S^{\prime}\right|=|S|-(p-q)=\delta(G-B)+q$. Now choose an arbitrary subset $I^{\prime}$ of $I$ of size $\delta(G-B)+k-q$ containing all vertices from $X \cup Y$ that exists due to (v) and (vi). We 


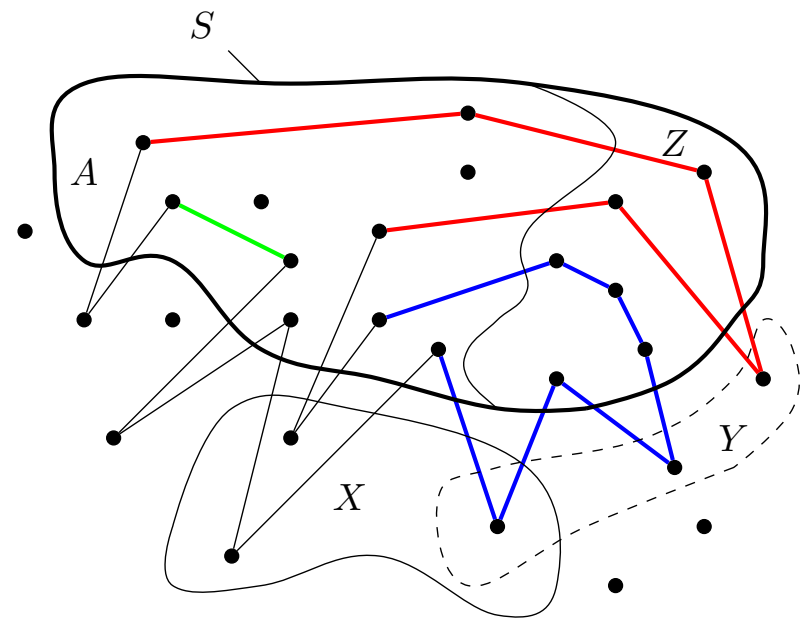

Figure 6: Illustration of how a cycle forms a path cover $\mathcal{P}$ from Lemma 13 . Edges belonging to different paths are colored with different colors. Vertices in $A$ that have no incident colored edge are covered by zero-length paths in $\mathcal{P}$.

consider $H=G\left[S^{\prime} \cup I^{\prime}\right]$. Notice that $|V(H)|=\left|S^{\prime}\right|+\left|I^{\prime}\right|=2 \delta(G-B)+k$. We claim that graph $H$ contains a Hamiltonian cycle. Clearly, this suffices for the proof, because the length of such a cycle is $2 \delta(G-B)+k$ and it contains all the vertices of $X \cup Z$ as required.

Let $H^{\prime}$ be the graph obtained from $H$ be the deletion of edges $e \in H\left[S^{\prime}\right]$ that are not included in the paths of $\mathcal{P}^{\prime}$. It is straightforward to see that it is sufficient to show that $H^{\prime}$ has a Hamiltonian cycle. By construction, $H^{\prime}\left[S^{\prime}\right]$ is the union of paths that are subpaths of the elements of $\mathcal{P}^{\prime}$. By (ii), no path of $\mathcal{P}^{\prime}$ has an endpoint in $Y$. Since $\mathcal{P}^{\prime}$ consists of paths with endpoints in $S^{\prime}$ covering $S^{\prime} \cup Y$ and there is no edges between vertices in $Y$, removal of each vertex from $Y$ breaks one path into two. Hence, the number of disjoint paths forming $H^{\prime}\left[S^{\prime}\right]$ is exactly $\left|\mathcal{P}^{\prime}\right|-|Y|=$ $\delta(G)+k-q-|Y|+|Y|=\left|I^{\prime}\right|$. This implies that the graph $H^{\prime \prime}$ obtained from $H^{\prime}$ by making every pair of distinct vertices of $I^{\prime}$ adjacent has a Hamiltonian cycle if and only if the same holds for $H^{\prime}$, because no Hamiltonian cycle of $H^{\prime \prime}$ cannot contain an edge $u v$ with $u, v \in Y$. Otherwise, such a cycle would cover $S^{\prime}$ by less than $\left|I^{\prime}\right|$ paths. Now the degree of each vertex from $I^{\prime}$ in $H^{\prime \prime}$ is at least $\delta(G-B)-(p-q)+\left|I^{\prime}\right|-1=2 \delta(G-B)+k-p-1$.

Take a vertex $v \in S^{\prime} \backslash Z$. Then $v \in A$ and, by the definition of $A, v$ has at least $p+1$ neighbors in $X \subseteq I^{\prime}$. Hence it has at least $p+1$ neighbors in $H^{\prime \prime}$. Therefore, the sum of vertex degrees of a vertex from $S^{\prime} \backslash Z$ and a vertex from $I^{\prime}$ in $H^{\prime \prime}$, is at least $2 \delta(G-B)+k=\left|V\left(G^{\prime}\right)\right|$. We construct $H^{\prime \prime \prime}$ from $H^{\prime \prime}$ by making adjacent every pair of vertices $u$ and $v$ with $u \in I^{\prime}$ and $v \in S^{\prime} \backslash Z$. Theorem 9 implies that $H^{\prime \prime \prime}$ has a Hamiltonian cycle if and only if $H^{\prime \prime}$ has a Hamiltonian cycle.

Finally, we construct a Hamiltonian cycle in $H^{\prime \prime \prime}$ using the paths of $\mathcal{P}^{\prime}$. For this, recall that each path of $\mathcal{P}^{\prime}$ has its endpoints in $A$ by (ii). Notice that there are exactly $\left|I^{\prime} \backslash Y\right|=\delta(G-B)+$ $k-q-|Y|$ vertices of $I^{\prime}$ that are not covered by the paths. Since the number of paths in $\mathcal{P}^{\prime}$ is $\delta(G-B)+k-q-|Y|$ and every endpoint of a path is adjacent to every vertex of $I^{\prime} \backslash Y$, it is straightforward to see that we can construct a Hamiltonian cycle joining the paths of $\mathcal{P}^{\prime}$ via the vertices of $I^{\prime} \backslash Y$.

Thus we conclude that $H^{\prime \prime \prime}$ has a Hamiltonian cycle. This implies that $H$ has a Hamiltonian cycle and competes the proof.

By Lemma 13 , to find a cycle of length $2 \delta(G-B)+k$ in $G$ containing all vertices in $C \cup Z$, 
it suffices to identify a path cover $\mathcal{P}$. Such a path cover can be computed by making use of color-coding. More precisely.

Lemma 14. Given $G, B, S, k$, and $X, A, Z$ defined in the same way as in Lemma 13, the existence of $Y$ and a path cover $\mathcal{P}$ of $G[S \cup Y]$ satisfying (i)-(vi) can be determined in $2^{\mathcal{O}(p)} \cdot n^{\mathcal{O}(1)}$ running time.

Proof. Because $p<\delta(G-B) / 8$ and $|X| \geq \delta(G-B)-3 p,|Z|<2 p$ by Lemma 12 . Then we are looking for $Y \subseteq I$ with $|Y| \leq 2|Z|<4 p$ by (iv). Also by (i), $\frac{k}{2} \leq q \leq p$. We assume without loss of generality that $q$ and the cardinality $r$ of $Y$ are fixed, as an algorithm can iterate over all $\mathcal{O}\left(p^{2}\right)$ possible pairs of these values in an outer loop. We also assume that (vi) holds for the given value of $q$. The algorithm is now to find a set of disjoint paths $\mathcal{P}$ covering all vertices in $S$ and a set $Y \subseteq I$ of size $r$. Since Lemma 13 requires an upper bound (v) on $|X \cup Y|$, we will aim to maximize $|X \cap Y|$, i.e. the number of vertices from $X$ used by the paths of $\mathcal{P}$.

As the paths of $\mathcal{P}$ cover exactly $|S|+|Y|$ vertices and their number is exactly $|S|+k-2 q-|Y|$ by (i), the total length of these paths is exactly $2|Y|+2 q-k \leq 10 p$. This allows us to deal with a bounded number of paths of positive length. By (ii), there is no path in $\mathcal{P}$ with an endpoint in $Z \cup Y$. In particular, this means that all paths of zero length are vertices in $A$ and the endpoint of nontrivial paths are in $A$. Each nontrivial path has exactly two endpoints in $A$. Then, because the total number of path $\mathcal{P}$ is $|S|+k-2 q-|Y|$, the number of nontrivial paths $t$ is at most $|A|-|\mathcal{P}|=|A|-(|S|+k-2 q-|Y|)=|Y|-|Z|+2 q-k \leq 6 p$. Note also that because $|\mathcal{P}|=|S|+k-2 q-|Y|$, the nontrivial paths should cover exactly $s=|S|+k-2 q-|Y|-t$ vertices of $A$ and they should leave uncovered at least $p-q$ vertices of $A$ to satisfy (iii). Clearly, $s \leq 20 p$, because the total length of the nontrivial paths is at most $10 p$. Thus, our task is reduced to deciding whether there is a set $Y \subseteq I$ of size $r \leq 4 p$ and a family of $t \leq|Y|-|Z|+2 q-k \leq 6 p$ disjoint nontrivial paths $\mathcal{P}^{\prime}$ such that

(a) the endpoints of the paths of $\mathcal{P}^{\prime}$ are in $A$,

(b) the paths cover the vertices of $Y$ and exactly $s=|S|+k-2 q-|Y|-t \leq 20 p$ vertices of $A$, and they leave uncovered at least $p-q$ vertices of $A$,

(c) subject to (a)-(b), $|Y \cap X|$ is maximum.

The color-coding technique of Alon, Yuster, and Zwick AYZ95 is a standard tool for solving problems of this type. Since the approach is standard (see, e.g, the book $\mathrm{CFK}^{+} 15 \mathrm{a}$, Chapter 5]), we only briefly sketch the algorithm. In the same way as in the proof of Theorem 4, we give a sketch of a randomized Monte Carlo algorithm and then explain how it can be derandomized.

For each positive integer $t \leq|Y|-|Z|+2 q-k$, we verify whether there are $Y$ and $\mathcal{P}^{\prime}$ satisfying (a) and (b) and find the maximum size of $|X \cap Y|$. After iterating over all possible values of $t$, the algorithm returns a solution that gives the maximum value of $X \cap Y$. For a given $t$, we compute $s=|S|+k-2 q-|Y|-t$ and verify whether $|A|-s \geq p-q$. We discard the current choice of $t$ if $|A|-s<p-q$. From now we assume that the value of $t$ is fixed and $|A|-s \geq p-q$.

We use the following randomized procedure. We color the vertices of $I$ by $r=|Y|$ distinct colors uniformly at random and then the vertices of $A$ are colored uniformly at random with another set of $s$ distinct colors. We also assume that the vertices of $Z$ are colored as well by pairwise distinct colors that are different from the colors used for $I$ and $A$. We denote by $C_{I}, C_{A}$, and $C_{Z}$ the sets of colors used to color $I, A$, and $Z$, respectively. Let also $C=C_{I} \cup C_{A} \cup C_{Z}$. Clearly, $|C|=\mathcal{O}(p)$. We say that $Y \subseteq I$ and a set of disjoint nontrivial paths $\mathcal{P}^{\prime}$ satisfying (a) and (b) is a coloful solution if the vertices of the paths are colored by distinct colors. 
The main steps of our algorithm either finds the maximum $|X \cap Y|$ for a colorful solution or reports that a colorful solution does not exist.

For a set of colors $R \subseteq C$, denote by $\alpha(R)$ the maximum number of vertices of $X$ that can be covered by a nontrivial path $P$ with $|R|$ vertices such that their the endpoint are in $A$ and the vertices of $P$ are colored by distinct colors from $R$; we assume that $\alpha(R)=-\infty$ if such a path does not exist. We observe that for every $R \subseteq C$, the value of $\alpha(R)$ can be computed in $2^{\mathcal{O}(p)} \cdot n^{\mathcal{O}(1)}$ time by a straightforward modification of the standard dynamic programming algorithm for finding a colorful $|R|$-path (see [AYZ95] and $\left[\mathrm{CFK}^{+} 15 \mathrm{a}\right.$, Chapter 5]). It is easy to incorporate the condition that the endpoits are in $A$. To maximize the number of vertices of $X$ used by a path, we can assume that the vertices of $X$ are of weight one and the vertices of $V(G) \backslash X$ are given zero weights. Then we use the variant of the algorithm that finds a colorful path of maximum weight. From now, we assume that we are given the table of values of $\alpha(R)$ for all $R \subseteq C$. Note that this table of size $2^{\mathcal{O}(p)}$ can be constructed in $2^{\mathcal{O}(p)} \cdot n^{\mathcal{O}(1)}$ time.

Let $R \subseteq C$, and $\ell \leq t$ be a positive integer. Denote by $\beta(R, \ell)$ the maximum number of vertices of $X$ that can be covered by exactly $\ell$ nontrivial path with $|R|$ vertices in total such that their endpoint are in $A$ and the vertices of the paths are colored by distinct colors from $R$; we assume that $\beta(R, \ell)=-\infty$ if such paths do not exist; in particular $\beta(R, \ell)=-\infty$ if $|R| \leq 1$. It is straightforward to see that $\beta(R, 1)=\alpha(R)$ for every $R \subseteq C$. To compute $\beta(R, \ell)$ for $\ell>1$, we use the following straightforward recurrence for $|R| \geq 2$.

$$
\beta(R, \ell)=\max \left\{\alpha\left(R^{\prime}\right)+\beta\left(R \backslash R^{\prime}, \ell-1\right) \mid \emptyset \neq R^{\prime} \subset R\right\} .
$$

We use (3) to compute the table of values of $\beta(R, t)$ for all nonempty $R \subseteq C$. Because $|C|=\mathcal{O}(p)$, computing the table can be done in $2^{\mathcal{O}(p)} \cdot n^{\mathcal{O}(1)}$ time.

By the choice of $C_{I}, C_{A}$ and $C_{Z}$, we have that $\beta(C, t)$ is the maximum number of vertices of $X$ that can be covered by a colorful solution, and $\beta(C, t)=-\infty$ if there is no colorful solution.

To obtain an optimum (non-colorful) solution, we define $N=\left\lceil e^{s+t}\right\rceil \geq \frac{r^{r} \cdot s^{s}}{r ! \cdot s !}$ and iterate the randomized procedure $N$ times. Then the algorithm returns a solution that gives the maximum value $|X \cap Y|$ over all coloful solution or reports that there is no solution if the algorithm fails to find a colorful solution in every iteration.

Suppose that $Y \subseteq I$ of size $r$ and $\mathcal{P}^{\prime}$ of size $t$ satisfy (a) and (b) and provide the maximum value of $|X \cap Y|$. Then with probability at least $\frac{r !}{r^{r}}$, the vertices of $Y$ are colored by distinct colors from $C_{I}$ by a random coring. Similarly, with probability at least $\frac{s !}{s^{s}}$, the $s$ vertices of $A$ covered by the paths of $\mathcal{P}^{\prime}$ are colored by distinct colors of $C_{A}$. Then with probability at least $\frac{r ! \cdot s !}{r^{r} \cdot s^{s}}$, the vertices of the paths of $\mathcal{P}^{\prime}$ are colored by distinct colors. Respectively, the probability that this does not holds, that is, there are at least two vertices of the same color, is at most $\left(1-\frac{r ! \cdot s !}{r \cdot \cdot s}\right)$. By the choice of $N$, we obtain that the probability that for every iteration, at least two vertices of paths of $\mathcal{P}$ have the same color, is at most $\left(1-\frac{r ! \cdot s !}{r^{r} \cdot s^{s}}\right)^{N} \leq e^{-1}$. Thus, the probability that the randomized algorithm fails to return an optimum solution is at most $e^{-1}<1$.

To evaluate the running time, recall that the tables of values of $\alpha(\cdot)$ and $\beta(\cdot, t)$ can be computed in $2^{\mathcal{O}(p)} \cdot n^{\mathcal{O}(1)}$ time. Since $r<4 p$ and $s \leq 20 p, N=2^{\mathcal{O}(p)}$ and, therefore, the total running time is $2^{\mathcal{O}(p)} \cdot n^{\mathcal{O}(1)}$.

To derandomize the algorithm, we use the standard technique (see [AYZ95] and $\mathrm{CFK}^{+} 15 \mathrm{a}$, Chapter 5]). For given $r$ and $s$, we construct the $(|I|, r)$ and $(|A|, s)$-perfect hash families of the functions $\mathcal{F}_{I}$ and $\mathcal{F}_{A}$, respectively, of sizes $e^{r} r^{\mathcal{O}(\log k)} \cdot \log |I|$ and $e^{s} s^{\mathcal{O}(\log s)} \cdot \log |A|$, respectively, using the results of Naor, Schulman, and Srinivasan [NSS95]. These families can be constructed in time $2^{\mathcal{O}(p)} \cdot n \log n$. Then we replace the random colorings of $I$ and $A$ by the functions from $\mathcal{F}_{I}$ and $\mathcal{F}_{A}$, respectively, and iterate the main step over all these functions. This gives deterministic $2^{\mathcal{O}(p)} \cdot n^{\mathcal{O}(1)}$ running time. 
To conclude the proof, note that algorithms finds the maximum possible size of $|X \cap Y|$ for $Y \subseteq I$ of size $r$ such that $S \cap Y$ can be covered by a set of paths $\mathcal{P}$ satisfying conditions (i)-(iv) and (vi) of Lemma 12. To verify (v), it is sufficient to check additionally whether $|X \cup Y| \leq \delta(G-B)+k-q$, by the maximality of $|X \cap Y|$. This concludes the proof.

Everything is settled for the proof of Theorem 6. For convenience, we restate the theorem here. Theorem 6. Long Dirac Cycle / Vertex Cover Above Degree is solvable in $2^{\mathcal{O}(p+|B|)}$. $n^{\mathcal{O}(1)}$ running time.

Proof. Let $(G, B, S, k)$ be a given instance of the problem. We assume without loss of generality that $B \subseteq S$; otherwise we can set $S:=S \cup B$ and $p:=p+|B \backslash S|$, which increases $p$ by at most $|B|$. Let $I=V(G) \backslash S$. Note that $G$ has no cycle longer than $2|S| \leq 2 \delta(G-B)+2 p$. In particular, if $k>2 p$, then the given instance is a no-instance. Therefore, we can assume that $k \leq 2 p$. If $\delta(G-B) \leq 8 p$, then $2 \delta(G-B)+k \leq 18 p$ and one can verify whether $G$ has a cycle of length $2 \delta(G-B)+k$ in $2^{\mathcal{O}(p)} \cdot n^{\mathcal{O}(1)}$ time using, e.g., the algorithm given by Zehavi [Zeh16]. From now on, we assume that $\delta(G-B)>8 p$. It is also convenient to assume that our aim is to verify the existence of a cycle of length exactly $2 \delta(G-B)+k$; for this we iterate over all possible values of the parameter from the initial given value of $k$ and $2 p$.

Also, if $p=0$, then $k=0$ and each vertex in $I$ is adjacent to all vertices in $S=\delta(G-B)$. Then $G$ contains all edges between $S$ and $I$, so a cycle of length at least $2 \delta(G-B)=2|S|$ exists in $G$ if and only if $|S| \geq|I|$ and $|S| \geq 2$. Thus, we can now assume that $p>0$.

If $|I|<\delta(G-B)+k-p$, then $(G, B, S, k)$ is a no-instance. Hence, we can assume that this is not the case. Our algorithm chooses an arbitrary $X \subseteq I$ of size $\delta(G-B)-3 p$. By Lemma 10, the algorithm can now look for a cycle of length $2 \delta(G-B)+k$ in $G$ containing all vertices from $X$.

Then we partition $S$ into two subsets $A$ and $Z$. The subset $A$ consists of all vertices in $S$ that have at least $p+1$ neighbors in $X$. The subset $Z$ consists of all other vertices in $S$. The running time of the procedure computing $Z$ is clearly polynomial. By Lemma 12 , the cardinality of $Z$ is at most $2 p$.

Before we can apply Lemmata 13 and 14 , we need to ensure that the cycle we are looking for contains all vertices from $Z$. To achieve that, we allow our algorithm to brute-force over all $2^{|Z|}=2^{\mathcal{O}(p)}$ options of how the cycle intersects $Z$. When an option is fixed, consider deleting from $G$ all vertices of $Z$ outside the fixed intersection. This can change the value of $p$, as $p=$ $|S|-\delta(G-B)$, and both $|S|$ and $\delta(G-B)$ may change after the deletion. As a consequence, the equality $|X|=\delta(G-B)-3 p$ could no longer hold, so we need to change $X$ correspondingly. Rewrite $\delta(G-B)-3 p=4 \delta(G-B)-3|S|$. Note that the removal of a single vertex of $Z$ from $G$ always decreases $|S|$ by one and can decrease $\delta(G-B)$ by at most one. Hence, the value $\delta(G-B)-3 p$ can only increase. Thus, after the deletion, to ensure $|X|=\delta(G-B)-3 p$, we add some vertices from $I$ to $X$. By Lemma 10 , the choice of these vertices can be arbitrary and we can be sure that there is a cycle containing $X$ while its intersection with $S$ remains the same. Each vertex in $A$ still has at least $p+1$ neighbors in $X$. Since $X$ now can containin some new vertices from $I$, a vertex in $Z$ may have at least $p+1$ neighbors in $X$. If such a vertex exists, we simply move it from $Z$ to $A$. Observe that the value of the parameter $p$ may be only decreased and the deletion does not violate the property $\delta(G-B)>8 p$. Note that the deletion operation discussed above also can imply an increment in $k$ as $\delta(G-B)$ can decrease. This is safe as Lemma 10 does not depend on the value of $k$ other than for estimating the length of the cycle.

After the intersection of the cycle with $Z$ is fixed and all vertices from $Z$ outside it are deleted from $G$, the algorithm finally employs the routine from Lemma 14 to find the path cover from 
Lemma 13 , hence to find the cycle. The total running time of the algorithm (under the assumption that $B \subseteq S$ ) is proportional to the number of sets $Z$, which is $2^{\mathcal{O}(p)}$, times the time required to compute the path cover for each of the sets, which is $2^{\mathcal{O}(p)} \cdot n^{\mathcal{O}(1)}$ by Lemma 14 . Hence the total running time is $2^{\mathcal{O}(p)} \cdot n^{\mathcal{O}(1)}$. Taking into account that to ensure the assumption that $B \subseteq S$ we may increase the initial value of $p$ by at most $|B|$, we conclude that the algorithm runs in $2^{\mathcal{O}(p+|B|)} \cdot n^{\mathcal{O}(1)}$ time.

\section{Finding almost Hamiltonian cycles}

This section is dedicated to the proof of Theorem 7 . To recall, the theorem states that given a graph $G$ with a set $B \subset V(G)$ and a parameter $k$ such that $|B| \leq k$ and $\delta(G-B) \geq \frac{n}{2}-k$, in time $2^{\mathcal{O}(k)} n^{\mathcal{O}(1)}$ we can find the longest cycle in $G$. Before we move on to prove the theorem itself, we show how to deal with the special case where there is a small separator in the graph, as it is an important subroutine in the main algorithm. Another key ingredient to the proof of Theorem 7 is our Long Dirac Cycle / Vertex Cover Above Degree result, presented in Section 7.

\subsection{Small separator lemma}

We show an algorithm for Almost Hamiltonian Dirac Cycle when there is a small (i.e. of size $\mathcal{O}(k))$ separator $B$ in $G$. Intuitively, the presense of a small separator makes the problem easier in the following sense. Each component of $G-B$ still has high minimal degree, slightly less than $\frac{n}{2}$. Thus, essentially, we must have exactly two components of size roughly $\frac{n}{2}$ in $G-B$, which means they are very dense. As was proven in $\left[\mathrm{FGL}^{+} 20 \mathrm{~b}\right]$, in this situation, we can always partition a component into paths that start and end at the given vertices, and span the whole component. We restate their result formally in the next lemma.

Lemma 15 (Lemma 1 in $\left[\mathrm{FGL}^{+} 20 \mathrm{~b}\right]$ ). Let $G$ be an $n$-vertex graph and $p$ be a positive integer such that $\delta(G) \geq \max \{5 p-3, n-p\}$. Let $\left\{s_{1}, t_{1}\right\}, \ldots,\left\{s_{r}, t_{r}\right\}, r \leq p$, be a collection of pairs of vertices of $G$ such that (i) $s_{i} \notin\left\{s_{j}, t_{j}\right\}$ for all $i \neq j, i, j \in\{1, \ldots, r\}$, and (ii) there is at least one index $i \in\{1, \ldots, r\}$ such that $s_{i} \neq t_{i}$. Then there is a family of pairwise vertex-disjoint paths $\mathcal{P}=\left\{P_{1}, \ldots, P_{r}\right\}$ in $G$ such that each $P_{i}$ is an $\left(s_{i}, t_{i}\right)$-path and $\cup_{i=1}^{r} V\left(P_{i}\right)=V(G)$, that is, the paths cover all vertices of $G$.

We note that the proof of Lemma 15 given in $\left[\mathrm{FGL}^{+} 20 \mathrm{~b}\right]$ is actually constructive. That is, there is a polynomial time algorithm that given $G, p$, and the respective set of pairs of vertices, returns the family of paths $\mathcal{P}$ from the statement of Lemma 15.

For simplicity, suppose there is a Hamiltonian cycle $C$ in $G$. The cycle induces a certain partition of $B$ into paths. On the other hand, if we are able to find any such path cover $\mathcal{P}$, we can construct the whole Hamiltonian cycle. Namely, on each component $H$ of $G-B$, we invoke Lemma 15 with a collection of pairs being a certain matching on ends of $\mathcal{P}$ belonging to $H$. In this way we connect the paths together while also visiting every vertex of $H$. If the pairs are selected in a certain way in both components, the union of all these parts will actually form a Hamiltonian cycle. We find the path cover itself with the help of dynamic programming and the color coding technique of Alon, Yuster, and Zwick AYZ95. In what follows, we prove the above in more detail.

Lemma 16. Let $G$ be a given 2 -connected graph on $n$ vertices and let $k \geq 0$ be a given integer. Let $B \subseteq V(G)$ be such that $|B| \leq k, \delta(G-B) \geq \frac{n}{2}-k$, and the graph $G-B$ is not connected. There is a $2^{\mathcal{O}(k)} \cdot n^{\mathcal{O}(1)}$ running time algorithm that finds the longest simple cycle in $G$. 
Proof. Assume $n \geq 12 k$, otherwise we invoke the general $2^{\mathcal{O}(n)}$ algorithm for the LONGEST CyCLE problem from Theorem 12 polynomial number of times to find the longest cycle in $G$.

First, observe that there are exactly two connected components in $G-B$. There must be at least two of them since $G-B$ is not connected. Suppose there are at least three components. Each of them contains a vertex of degree at least $\frac{n}{2}-k$ in $G-B$, therefore the size of each component is at least $\frac{n}{2}-k+1$. The total number of vertices is then at least $3 \frac{n}{2}-3 k+3=n+\left(\frac{n}{2}-3 k\right)+3>n$. This is a contradiction. From now on, let $H_{1}$ and $H_{2}$ be the two connected components of $G-B$.

Consider the longest cycle $C$ in $G$. Recall that by Theorem 14 the length of $C$ is at least $\min \{n-2 k, n-|B|\} \geq n-2 k$, thus it necessarily contains vertices from all of $H_{1}, H_{2}$ and $B$. We say that $C$ induces a path cover $\mathcal{P}$ of $B$, where $\mathcal{P}$ is the set of paths that $C$ forms when restricted to the edges incident to $B$. In other words, remove from $C$ all the edges that are not incident to $B$, and all the vertices that became isolated after that. The resulting collection of vertex-disjoint paths is the path cover $\mathcal{P}$. Note that $\mathcal{P}$ satisfies the following properties.

1. Every path $P \in \mathcal{P}$ starts and ends in $V(G) \backslash B$.

2. Each path $P \in \mathcal{P}$ has at least one vertex in $B$ and no two consecutive vertices in $V(G) \backslash B$.

3. The paths of $\mathcal{P}$ contain at most $3|B|$ vertices in total.

4. The number of paths in $\mathcal{P}$ that start and end in different components of $G-B$ is even and at least two.

Since for every vertex of $B$, its degree in $C$ is exactly two even when restricted to the edges incident to $B$, the property (1) follows. Each path goes through $B$, and two vertices in $V(G) \backslash B$ cannot be adjacent via an edge incident to $B$, thus (2) follows. Property (3) follows directly from property (2). Finally, (4) holds since $C$ must leave both $H_{1}$ and $H_{2}$ an even number of times. Moreover, if there are no paths in $\mathcal{P}$ that start and end in different components, $H_{1}$ and $H_{2}$ cannot be connected via $C$, thus $C$ is not a cycle of length at least $n-2 k$.

We call a set of vertex-disjoint paths in $G$ satisfying (1)-(4) a good path cover. Now we claim that any good path cover can be used to construct a long cycle in $G$, i.e. we can collect all the vertices of $V(G) \backslash B$ in a cycle by going along the paths in the cover. The proof is essentially by pairing endpoints of the paths carefully and then applying Lemma 15 to both $H_{1}$ and $H_{2}$. The illustration is shown in Figure 7 and the proof follows next.

Claim 10. There is a polynomial time algorithm that given a good path cover $\mathcal{P}$ finds a cycle of length $n-t$ in $G$, where $t$ is the number of vertices in $B$ not covered by the paths in $\mathcal{P}$.

Proof. Denote $\mathcal{P}=\left\{P_{1}, \ldots, P_{r}\right\}$, and for each $i \in\{1, \ldots, r\}$, denote the two ends of the path $P_{i}$ by $s_{i}$ and $t_{i}$. We may assume that the paths are ordered in a way that paths $P_{1}, \ldots, P_{a}$ lead from $H_{1}$ to $H_{2}$, paths $P_{a+1}, \ldots, P_{b}$ start and end in $H_{1}$, and paths $P_{b+1}, \ldots, P_{r}$ start and end in $H_{2}$, for certain integers $a$ and $b$, such that $1<a \leq b \leq r$, and $a$ is even by property (5). Additionally, for $i \in\{1, \ldots, a\}$ assume that $s_{i} \in V\left(H_{1}\right), t_{i} \in V\left(H_{2}\right)$.

Let $I$ be the set of internal vertices of paths in $\mathcal{P}$, let $H_{1}^{\prime}=H_{1}-I, H_{2}^{\prime}=H_{2}-I$. The graphs $H_{1}^{\prime}$ and $H_{2}^{\prime}$ are targets for applying Lemma 15 . By property (2), the size of $I \backslash B$ is at most $k$, thus $\delta\left(H_{1}^{\prime}\right) \geq \delta(G-B)-k=\frac{n}{2}-2 k$, and by the same argument $\delta\left(H_{2}^{\prime}\right) \geq \frac{n}{2}-2 k$.

Consider the following set $T_{1}$ of $b-\frac{a}{2}$ pairs of vertices in $H_{1}^{\prime}$. If $b=a$, the pairs are $\left\{s_{1}, s_{2}\right\}$, $\left\{s_{3}, s_{4}\right\}, \ldots,\left\{s_{a-1}, s_{a}\right\}$. If $b>a$, the pairs are $\left\{s_{2 i-1}, s_{2 i}\right\}$ for $1 \leq i<\frac{a}{2},\left\{s_{a-1}, s_{a+1}\right\},\left\{t_{j}, s_{j+1}\right\}$ for $a+1 \leq j<b$, and $\left\{t_{b}, s_{a}\right\}$.

Now, we apply Lemma 15 to the graph $H_{1}^{\prime}$, the set of pairs $T_{1}$, and we set the parameter $p$ to be $2 k$. Since pairs in $T_{1}$ are disjoint, and $\max \{5 p-3, n-p\}=\max \{10 k-3, n-2 k\} \leq \delta\left(H_{1}^{\prime}\right)$, all 


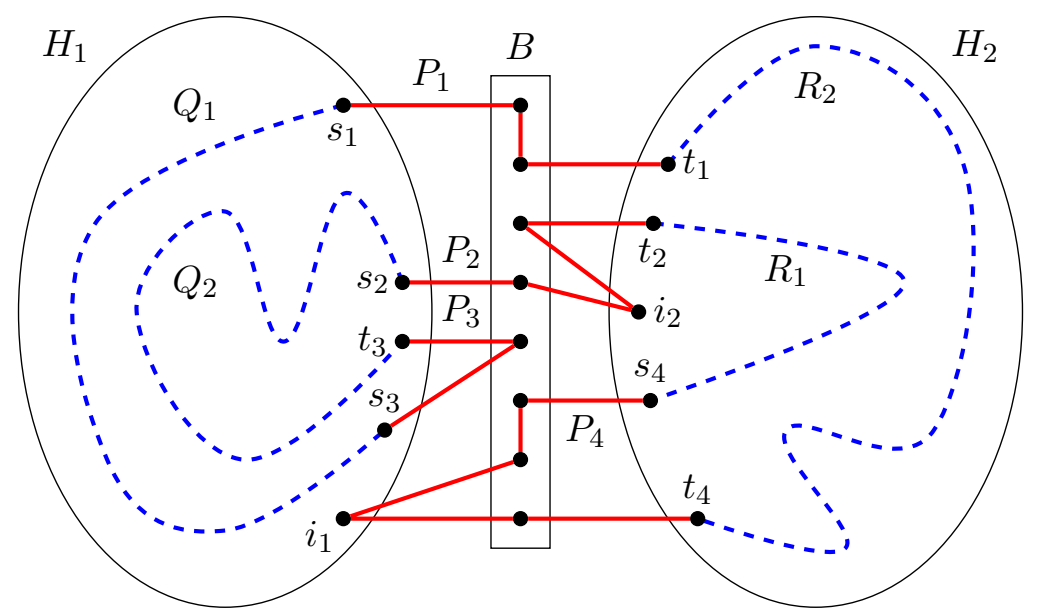

Figure 7: Reconstructing the cycle from the good path cover $\mathcal{P}=\left\{P_{1}, P_{2}, P_{3}, P_{4}\right\}$. The paths $Q_{1}$ and $Q_{2}$ are obtained by applying Lemma 15 to $H_{1}^{\prime}=H_{1}-\left\{i_{1}\right\}$, the same for the paths $R_{1}, R_{2}$ and the graph $H_{2}^{\prime}=H_{2}-\left\{i_{2}\right\}$. The resulting concatenation of paths is a Hamiltonian cycle in $G$.

conditions of the lemma are satisfied. Thus, there exist vertex-disjoint paths $Q_{1}, \ldots, Q_{b-\frac{a}{2}}$ that have the respective endpoints from $T_{1}$ and cover all vertices of $H_{1}^{\prime}$.

We deal with $H_{2}^{\prime}$ similarly. We only need to connect $t_{1}, \ldots, t_{a}$ in a shifted way compared to $s_{1}, \ldots, s_{a}$, so that we obtain a cycle at the end. Consider the following set $T_{2}$ of $\frac{a}{2}+r-b$ pairs of vertices in $H_{2}^{\prime}$. If $b=r$, the pairs are $\left\{t_{2}, t_{3}\right\},\left\{t_{4}, t_{5}\right\}, \ldots,\left\{t_{a-2}, t_{a-1}\right\}$, and $\left\{t_{1}, t_{a}\right\}$. If $b<r$, the pairs are $\left\{t_{2 i}, t_{2 i+1}\right\}$ for $1 \leq i<\frac{a}{2},\left\{t_{a}, s_{b+1}\right\},\left\{t_{j}, s_{j+1}\right\}$ for $b+1 \leq j<r$, and $\left\{t_{1}, t_{r}\right\}$. Again, we apply Lemma 15 to the graph $H_{2}^{\prime}$, the set of pairs $T_{2}$, and $p=2 k$. We obtain vertex-disjoint paths $R_{1}, \ldots, R_{r-b+\frac{a}{2}}$ that have the respective endpoints from $T_{2}$ cover all vertices of $H_{2}^{\prime}$.

The resulting cycle $C$ with $V(C)=(V(G) \backslash B) \cup I$ is a cyclic concatenation of paths $P_{1}, \ldots$, $P_{r}, Q_{1}, \ldots, Q_{b-\frac{a}{2}}, R_{1}, \ldots, R_{r-b+\frac{a}{2}}$ in a certain order. Namely,

$$
C=P_{1} Q_{1} P_{2} R_{1} \cdots P_{a-1} Q_{\frac{a}{2}} P_{a+1} Q_{\frac{a}{2}+1} P_{a+2} \cdots P_{b} Q_{b-\frac{a}{2}} P_{a} R_{\frac{a}{2}} P_{b+1} \cdots P_{r} R_{r-b+\frac{a}{2}},
$$

where we understand the notation $P Q$ for paths $P$ and $Q$ with a common endpoint as their natural concatenation. Clearly, $C$ is a cycle, and it spans all the previously defined paths. By construction, these paths cover all vertices in $I, V\left(H_{1}^{\prime}\right)$, and $V\left(H_{2}^{\prime}\right)$, thus they cover all vertices in $V(G)$ except those vertices in $B$ that are not covered by $\mathcal{P}$.

Now it only remains to find a good path cover that covers the maximum number of vertices in $B$. By Claim 10, a good path cover immediately gives us a cycle of the corresponding length, and we have also showed that a long cycle in $G$ induces a good path cover.

To find the desired good path cover, first we observe that the number of vertices covered by the paths in the cover is at most $3|B|$ by property (3) of a good path cover. We proceed with a color-coding scheme using $r=3|B|$ colors: color each vertex in $B$ in its own color, and each vertex in $V(G) \backslash B$ randomly and independently in one of the remaining $r-|B|$ colors, with equal probability for each color. Denote this coloring by $c: V(G) \rightarrow\{1, \ldots, r\}$. Now we look for a colored good path cover, that is, a good path cover that covers at most one vertex of each color.

We find a colored good path cover with the help of dynamic programming. Define a state as a tuple $(C, v, i, \ell, p)$ where $C$ is a subset of $\{1, \ldots, r\}, v$ is a vertex in $V(G), i \in\{1,2\}, \ell \in\{1, \ldots, r\}$, and $p \in\{0,1, \ldots,|B|\}$. We call a state $(C, v, i, \ell, p)$ feasible if there exists a set of vertex-disjoint paths $\mathcal{P}=\left\{P_{1}, \ldots, P_{t}\right\}$ in $G$ such that the following holds. 
1. Every path $P_{1}, \ldots, P_{t-1}$ starts and ends in $V(G) \backslash B$ and has the length of at least three, $P_{t}$ starts in $V\left(H_{i}\right)$, ends in $v$, and its length is $\ell$.

2. No path $P \in \mathcal{P}$ has two consecutive vertices in $V(G) \backslash B$.

3. The paths in $\mathcal{P}$ cover exactly one vertex of each color in $C$, and no vertices of other colors.

4. The number of paths in $\left\{P_{1}, \ldots, P_{t-1}\right\}$ that start and end in different components of $G-B$ is exactly $p$.

Note that $\mathcal{P}$ in the definition of a feasible state is essentially an "unfinished" good path cover that agrees with the state $(C, v, i, \ell, p)$. Our goal now is to compute the set of all feasible states $S$. We start by setting

$$
S_{1}=\left\{(\{c(v)\}, v, 1,1,0): v \in V\left(H_{1}\right)\right\} \cup\left\{(\{c(v)\}, v, 2,1,0): v \in V\left(H_{2}\right)\right\} .
$$

These are our initial states, corresponding to sets containing one path of length one. Trivially, each such state is feasible, and these are all feasible states that use exactly one color. Next, for each $j$ in $\{1, \ldots, r-1\}$, we show how to compute the set of feasible states $S_{j+1}$ of size $j+1$ from $S_{j}$, the set of feasible states of size $j$. Here by the size of the state $(C, v, i, \ell, p)$ we mean $|C|$, the number of colors used, which is the same as the total number of vertices covered by any set of paths corresponding to the state.

To compute $S_{j+1}$ from $S_{j}$, we iterate over all states in $S_{j}$ and try to extend each of them by an additional vertex. Intuitively, we either extend the unique unfinished path corresponding to the state, or declare it finished and start a new path. Fix a state $(C, v, i, \ell, p) \in S_{j}$, there is a set of paths $\mathcal{P}=\left\{P_{1}, \ldots, P_{t}\right\}$ satisfying the feasibility definition for $(C, v, i, \ell, p)$. Consider each $u \in N_{G}(v)$ such that $c(u) \notin C$. If both $v$ and $u$ are not in $B$, we do nothing. Otherwise, add to $S_{j+1}$ the state $(C \cup c(u), u, i, \ell+1, p)$. Clearly, the size of this state is $j+1$, and it is easy to verify that the set of paths $\mathcal{P}^{\prime}=\left\{P_{1}, \ldots, P_{t} u\right\}$ satisfies the feasibility definition for $(C \cup c(u), u, i, \ell+1, p)$.

For the "new path" kind of extending $(C, v, i, \ell, p)$ with $\ell>2$, consider each vertex $u \in V(G) \backslash B$ such that $c(u) \notin C$. If $v \in B$ do nothing, otherwise add to $S_{j+1}$ the state $\left(C \cup c(u), u, i^{\prime}, 1, p^{\prime}\right)$, where $i^{\prime}$ is such that $u \in H_{i^{\prime}}$ and $p^{\prime}=p$ if $v \in V\left(H_{i}\right)$, or $p^{\prime}=p+1$ if $v \notin V\left(H_{i}\right)$. To see that this state is feasible, consider the set of paths $\mathcal{P}^{\prime}=\left\{P_{1}, \ldots, P_{t}, u\right\}$. Indeed, every path among $P_{1}, \ldots$, $P_{t-1}$ starts and ends in $V(G) \backslash B$, and $P_{t}$ as well, since $v \in V(G) \backslash B$. The length of $P_{t}$ is $\ell$ so at least three, and for $P_{1}, \ldots, P_{t-1}$ this holds by feasibility of the original state. The last path $u$ starts in $V\left(H_{i^{\prime}}\right)$ by definition of $i^{\prime}$, ends in $u$, and has the length of one. Properties (2) and (3) are preserved automatically. The value $p^{\prime}$ reflects exactly how $p$ is changed with respect to the newly finished path $P_{t}$.

Now we show that $S_{j+1}$ contains all feasible states of size $j+1$, provided that $S_{j}$ contains all feasible states of size $j$. Consider a state $\left(C^{\prime}, u, i^{\prime}, \ell^{\prime}, p^{\prime}\right) \in S_{j+1}$ and a corresponding set of paths $\mathcal{P}^{\prime}=\left\{P_{1}, \ldots, P_{t}\right\}$. Recall that $\left|P_{t}\right|=\ell^{\prime}$, if $\ell^{\prime}>1$, consider a state $\left(C, v, i^{\prime}, \ell^{\prime}-1, p^{\prime}\right)$ where $v$ is the previous vertex to $u$ in $P_{t}, C=C^{\prime} \backslash\{c(u)\}$. Observe that $\left(C, v, i^{\prime}, \ell^{\prime}-1, p^{\prime}\right)$ is feasible as witnessed by the set of paths $\mathcal{P}=\left\{P_{1}, \ldots, P_{t}^{\prime}\right\}$ where $P_{t}^{\prime}$ is $P_{t}$ without its last vertex $u$. Since $u \in N_{G}(v), c(u) \notin C$, and $v$ and $u$ are not simultaneously in $V(G) \backslash B$ by property (2) for $\mathcal{P}^{\prime}$, the state $\left(C^{\prime}, u, i^{\prime}, \ell^{\prime}, p^{\prime}\right)$ is added to $S_{j+1}$ when the algorithm considers extending the state $\left(C, v, i^{\prime}, \ell^{\prime}-1, p^{\prime}\right) \in S_{j}$ by the vertex $u$. If $\ell^{\prime}=1$, consider a state $(C, v, i, \ell, p)$ where $v$ is one of the endpoints of $P_{t-1}, i$ is the index of the component of the other endpoint of $P_{t-1}, \ell=\left|P_{t-1}\right|$, $C=C^{\prime} \backslash\{c(u)\}$, and $p$ is either $p^{\prime}$ or $p^{\prime}-1$, depending on whether $v$ belongs to $H_{i}$ or not. The set of paths $\left\{P_{1}, \ldots, P_{t-1}\right\}$ witnesses the feasibility of $(C, v, i, \ell, p)$, and thus $\left(C^{\prime}, u, i^{\prime}, \ell^{\prime}, p^{\prime}\right)$ is added to $S_{j+1}$ on the corresponding "new path" step. 
Therefore, we have shown that for each $j$ in $\{1, \ldots, r-1\}$, we correctly compute the set $S_{j+1}$ from $S_{j}$, so in the end we have the sets $S_{j}$ of feasible states of size $j$, for each $j \in\{1, \ldots, r\}$. Finally, we consider a subset $\mathcal{C}$ of the feasible states $(C, v, i, \ell, p) \in \bigcup_{j=1}^{r} S_{j}$, such that $v \notin B, \ell>2$, and $p^{\prime}$ is at least 2 and even, where $p^{\prime}=p$ if $v \in H_{i}$ and $p^{\prime}=p+1$ if $v \notin H_{i}$. Note that $\mathcal{C}$ is not empty since a long cycle in $G$ guaranteed by Theorem 14 induces a good path cover, and thus a feasible state of the form above. From $\mathcal{C}$, we pick a state maximizing $|C \cap\{1, \ldots,|B|\}|$. The set of paths $\left\{P_{1}, \ldots, P_{t}\right\}$ corresponding to this state is a good path cover in $G$ that covers the maximum number of vertices in $B$. Note that the actual good path cover may be found by the usual means of backtracking in dynamic programming. Together with Claim 10 this concludes the algorithm, and the proof of its correctness.

Running time analysis. In the dynamic programming part, the number of states is at most $2^{r} \cdot n \cdot 2 \cdot r \cdot(k+1)$. While considering a state, we update $\mathcal{O}(n)$ other states, thus the total running time of the dynamic programming subroutine is $2^{\mathcal{O}(k)} n^{\mathcal{O}(1)}$. For a fixed long cycle $C$ in $G$, the probability that we guess the coloring that assigns different colors to all vertices of the induced by $C$ good path cover, is at least $e^{-r}$, since there are at most $r$ vertices in the good path cover. By performing $\left\lceil e^{r}\right\rceil$ iterations of the color coding subroutine, we amplify the success probability to at least $1-\left(1-e^{r}\right)^{e^{r}} \geq 1-e^{-1}$. Therefore, we obtain a Monte Carlo algorithm with constant success probability and running time $\mathcal{O}\left(k^{2} \cdot e^{3 k} \cdot 2^{3 k} \cdot n^{2}\right)=\mathcal{O}\left(2^{\mathcal{O}(k)} n^{\mathcal{O}(1)}\right)$. Finally, the algorithm could be derandomized in the standard fashion by using perfect hash families [NSS95].

\subsection{Main theorem}

Now we move on to Theorem 7, the main result of this section. We restate the theorem here for convenience of the reader.

Theorem 7. Let $G$ be a given 2-connected graph on $n$ vertices and let $k$ be a given integer. Let $B \subseteq V(G)$ be such that $|B| \leq k$ and $\delta(G-B) \geq \frac{n}{2}-k$. There is a $2^{\mathcal{O}(k)} \cdot n^{\mathcal{O}(1)}$ running time algorithm that finds the longest cycle in $G$.

Proof. First, we may assume that $n>40 k$, otherwise the problem can be solved by the classical $2^{\mathcal{O}(n)}$ algorithm for LONGEST CYCLE. Instead of proving the theorem directly, we show that there exists an algorithm that in time $2^{\mathcal{O}(k)} \cdot n^{\mathcal{O}(1)}$ either

1. finds the longest cycle in $G$, or

2. finds a vertex cover of $G$ of size at most $\frac{n}{2}+9 k$, or

3. finds a set $B^{\prime} \supseteq B$ of size at most $35 k$ such that $G-B^{\prime}$ is not connected.

We say that (1)-(3) are the terminal states of the algorithm.

If state (3) is reached, we simply invoke the algorithm from Lemma 16 with the respective separating set $B^{\prime}$ of size at most $35 k$. This gives us immediately the longest cycle in $G$. Similarly, reaching terminal state (2) also suffices to solve the problem, as shown in the next claim.

Claim 11. If terminal state (2) is reached, the longest cycle in $G$ can be found in $2^{\mathcal{O}(k)} \cdot n^{\mathcal{O}(1)}$ time.

Proof of Claim 11. Denote the obtained vertex cover of $G$ of size at most $\frac{n}{2}+9 k$ by $S$. We would like to invoke the algorithm given by Theorem 6 , but we are not guaranteed that the longest cycle in $G$ has length of the form $2 \delta(G-B)+k^{\prime}$ for $k^{\prime} \geq 0$. 
By Theorem 14, we have that there is a cycle of length at least $\min \{2 \delta(G-B), n-|B|\}$ in $G$, as $G$ is 2-connected. We aim to achieve $2 \delta(G-B) \leq n-|B|$. Each vertex in $G-B$ has at most $|S|$ neighbours. Take the vertex in $G-B$ with smallest degree. It has at least $\frac{n}{2}-k>19 k$ neighbours in $G-B$. Obtain $B^{\prime}$ by adding $19 k$ neighbours of this vertex in $G-B$ to $B$. We have that $\delta\left(G-B^{\prime}\right)=\delta(G-B)-19 k$, and $G-B^{\prime}$ still contains at least one vertex.

Note that $\delta\left(G-B^{\prime}\right) \leq|S|-19 k \leq \frac{n}{2}-10 k \leq \frac{n}{2}-\frac{\left|B^{\prime}\right|}{2}$, as $\left|B^{\prime}\right| \leq 20 k$, so $2 \delta\left(G-B^{\prime}\right) \leq n-\left|B^{\prime}\right|$. Thus, by Theorem 15, the length of the longest cycle in $G$ is of form $2 \delta\left(G-B^{\prime}\right)+k^{\prime}$ for $k^{\prime} \geq 0$. The size of the vertex cover $S$ is at most $\frac{n}{2}+9 k \leq \delta(G-B)+10 k=\delta\left(G-B^{\prime}\right)+29 k$.

Recall that Theorem 6 provides a $2^{\mathcal{O}\left(p+k^{\prime}\right)} \cdot n^{\mathcal{O}(1)}$-time algorithm that finds a cycle of length at least $2 \delta\left(G-B^{\prime}\right)+k^{\prime}$ given a vertex cover of $G$ of size $\delta\left(G-B^{\prime}\right)+p$, if there is any. By trying all possible $k^{\prime}$ from $n-2 \delta\left(G-B^{\prime}\right) \leq 40 k$ to zero, we find the longest cycle in $G$ in time $2^{\mathcal{O}(k)} \cdot n^{\mathcal{O}(1)}$ as $p \leq 29 k$. By Theorem 14 there is a cycle of length at least $2 \delta\left(G-B^{\prime}\right)$ in $G$, thus invoking Theorem 6 with $k^{\prime}=0$ necessarily provides us with a cycle.

Therefore, in what follows we assume that reaching any of the terminal states solves the problem immediately.

Now consider a cycle $C$ of maximum length in $G$. Identically to the proof of Lemma 15, $C$ induces a path cover of a subset of $B$. Namely, in this proof, we call a set of vertex-disjoint paths $\mathcal{P}$ in $G$ a good path cover if $\mathcal{P}$ satisfies the following properties.

1. Every path $P \in \mathcal{P}$ starts and ends in $V(G) \backslash B$.

2. Each path $P \in \mathcal{P}$ has at least one vertex in $B$ and no two consecutive vertices in $V(G) \backslash B$.

3. The paths of $\mathcal{P}$ contain at most $3|B|$ vertices in total.

Note that this definition is the same as in Lemma 15, except for the property (4) there. Intuitively, we do not need it in this lemma since we may now assume that $G-B$ is connected. Since the current definition is strictly less restrictive, it follows immediately from the proof of Lemma 15 that

- for each $0 \leq t \leq|B|$, if there is a cycle of length $n-t$ in $G$, there is also a good path cover in $G$ that covers all but $t$ vertices of $B$,

- in time $2^{\mathcal{O}(k)} n^{\mathcal{O}(1)}$ we can find a good path cover $\mathcal{P}$ that covers the maximum number of vertices in $B$, by the combination of color coding and dynamic programming.

Note that the empty set is a good path cover, thus a good path cover always exists.

So for the rest of the proof we deal with the case where we have computed a good path cover $\mathcal{P}$ of $G$, possibly an empty one. Denote by $r$ the number of paths in $\mathcal{P}$, and by $B^{\prime}$ the set of vertices covered by paths in $\mathcal{P}$ together with the rest of vertices of $B$. By definition, $B \subset B^{\prime}$, and by property (2) of a good path cover $\left|B^{\prime}\right| \leq 3 k$. If $G-B^{\prime}$ is not connected, we have a small separator: the algorithm outputs $B^{\prime}$ and stops, reaching terminal state (3). If $G-B^{\prime}$ is not 2-connected, we add to $B^{\prime}$ an arbitrary cut vertex of $G-B^{\prime}$ and return $B^{\prime}$. Thus, from now on we may assume that $G-B^{\prime}$ is 2 -connected. The minimum degree of $G-B^{\prime}$ is at least

$$
\delta\left(G-B^{\prime}\right) \geq \frac{n}{2}-k-\left|B^{\prime} \backslash B\right| \geq \frac{n-\left|B^{\prime} \backslash B\right|}{2}-2 k>\frac{n-\left|B^{\prime}\right|+2}{3},
$$

since $\left|B^{\prime} \backslash B\right| \leq 2 k$ and $n>16 k$. By Theorem 11, in time $\mathcal{O}\left(n^{3}\right)$ we find either a Hamiltonian cycle $C_{0}$ in $G-B^{\prime}$, or an independent set of size $\delta\left(G-B^{\prime}\right)+1$. If an independent set is found, its complement in $G-B^{\prime}$ together with $B^{\prime}$ is a vertex cover of $G$ of size at most $\frac{n}{2}+k+2\left|B^{\prime}\right| \leq \frac{n}{2}+7 k$. In this case we output the vertex cover and stop, reaching terminal state $(2)$. 
Otherwise, we have a Hamiltonian cycle $C_{0}$ in $G-B^{\prime}$. Now, we iteratively insert the paths of $\mathcal{P}=\left\{P_{1}, \ldots, P_{r}\right\}$ into the cycle. Namely, for each $i \in\{1, \ldots, r\}$ we prove that given a cycle $C$ that contains exactly the vertices of the cycle $C_{0}$ and the paths $P_{1}, \ldots, P_{i-1}$, we can either modify the cycle $C$ such that it satisfies the same property for $i+1$, i.e. contains the vertices of the path $P_{i}$ as well, or reach one of the terminal states. Clearly, applying the above for each $i \in\{1, \ldots, r\}$, starting from the cycle $C_{0}$, proves the theorem. Thus from now on we focus on this statement.

Consider the path $P_{i}$ and the obtained cycle $C$ that contains all vertices of $C_{0}$ and $P_{1}, \ldots, P_{i-1}$. Denote the endpoints of $P_{i}$ by $s$ and $t$, observe that both $s$ and $t$ have at least $\frac{n}{2}-3 k$ neighbors on $C$. That holds since $s \notin B$, so $\operatorname{deg}_{G-B}(s) \geq \frac{n}{2}-k$, and at most $2 k$ vertices of $G$ belong to $B^{\prime} \backslash B$ and are neither on $C$ nor in $B$, analogously for $t$.

Denote by $C_{s}$ the set of neighbors of $s$ on $C$, and by $C_{t}$ the set of neighbors of $t$ on $C$. Consider a vertex $c_{s} \in C_{t}$ and a vertex $c_{t} \in C_{t}$. If $c_{s}$ and $c_{t}$ are next to each other on $C$ then we can immediately insert $P_{i}$ in $C$. If these vertices are not adjacent, but are at distance two on $C$ with a vertex $c^{\prime} \notin B$ between them, we do the following. Insert $P_{i}$ in $C$ by going from $c_{s}$ to $c_{t}$ through $P_{i}$ and not through $c^{\prime}$, denote the resulting cycle by $C^{\prime}$. The vertex $c^{\prime}$ is the only vertex that is in $V(C) \cup V\left(P_{i}\right)$, but not on $C^{\prime}$, thus we are done as long as we insert $c^{\prime}$ back in $C^{\prime}$. By the same argument as for $s$ and $t, c^{\prime}$ has at least $\frac{n}{2}-3 k$ neighbors on $C^{\prime}$. If there are two neighbors of $c^{\prime}$ on $C^{\prime}$ that are consecutive on $C^{\prime}$ again we can immediately insert $c^{\prime}$ in $C^{\prime}$, thus we assume this is not the case. Now on $C^{\prime}$ between every two consecutive neighbors of $c^{\prime}$ there is a group of at least one and possibly several non-neighbors of $c^{\prime}$. Since there are at least $\frac{n}{2}-3 k$ neighbors of $c^{\prime}$ on $C^{\prime}$, there are also at least $\frac{n}{2}-3 k$ such groups of consecutive non-neighbors. Since there are at most $\frac{n}{2}+3 k$ non-neighbors of $c^{\prime}$ on $C^{\prime}$, at most $6 k$ of the groups may contain more than one vertex. Thus at least $\frac{n}{2}-9 k$ groups consist of a single vertex, denote the set of all such vertices by $I$. Each vertex of $I$ is not adjacent to $c^{\prime}$, but both of its neighbors on $C^{\prime}$ are adjacent to $c^{\prime}$. We claim that if two vertices in $I$ are adjacent in $G$, there is a cycle that goes through $c^{\prime}$ and all vertices of $C^{\prime}$. Denote these vertices by $u$ and $v$, go from $c^{\prime}$ to a neighbor of $u$, then to $v$ along the arc of $C^{\prime}$ that does not contain $u$, then take the edge $u v$, and finally collect the rest of $C^{\prime}$ going from $u$ to a neighbor of $v$ and returning to $c^{\prime}$. If no two vertices in $I$ are adjacent in $G$, then $I$ is an independent set of size at least $\frac{n}{2}-9 k$ in $G$. Thus the complement of $I$ is a vertex cover of $G$ of size at most $\frac{n}{2}+9 k$, and we are in the terminal state $(2)$.

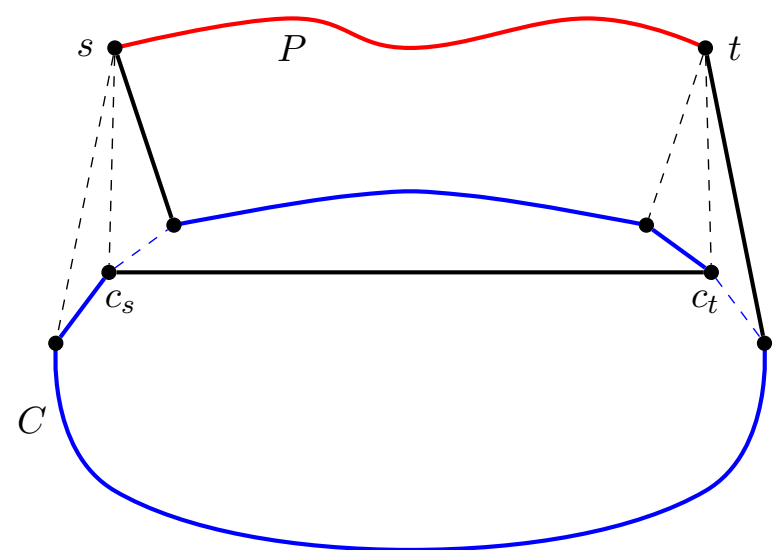

Figure 8: Inserting the path $P$ (in red) into the cycle $C$ (in blue) in the presence of an edge between an internal $s$-vertex $c_{s}$ and an internal $t$-vertex $c_{t}$. The resulting cycle is in solid.

Now we deal with the case where for every $c_{s} \in C_{s}$ and every $c_{t} \in C_{t}$, there is either a vertex of $B$ or at least two other vertices between them on $C$. First, we bound the number of common 
neighbors of $s$ and $t$ on $C$, denote $C_{s} \cap C_{t}$ by $C_{s t}$. Fix an ordering on $C$, and consider a vertex $u \in C_{s t}$ and the next vertex $v$ along the cycle that belongs to either $C_{s}$ or $C_{t}$. Between $u$ and $v$, there must be at least two vertices that belong to neither $C_{s}$ nor $C_{t}$, or a vertex of $B$. Thus with each vertex of $C_{s t}$ we can uniquely associate either two vertices of $V(C) \backslash C_{s} \backslash C_{t}$, or a vertex of $B$. We get that apart from the vertices of $C_{s t}, C_{s} \backslash C_{s t}$ and $C_{t} \backslash C_{s t}$, there are at least $2\left(\left|C_{s t}\right|-|B|\right)$ other vertices in $C$. Summing the sizes of these four disjoint sets together, we get

$$
\begin{aligned}
& \left|C_{s t}\right|+\left(\left|C_{s}\right|-\left|C_{s t}\right|\right)+\left(\left|C_{t}\right|-\left|C_{s t}\right|\right)+2\left(\left|C_{s t}\right|-|B|\right) \leq n \\
& \left|C_{s t}\right| \leq n-\left|C_{s}\right|-\left|C_{t}\right|+2|B| \leq 8 k
\end{aligned}
$$

since both $C_{s}$ and $C_{t}$ contain at least $\frac{n}{2}-3 k$ vertices, and the size of $B$ is at most $k$. From this bound, we also immediately get that the number of vertices on $C$ that are not adjacent to both $s$ and $t$ is at most $n-\left|C_{s}\right|-\left|C_{t}\right|+\left|C_{s t}\right| \leq 14 k$. Thus nearly all vertices of $C$, except for $\mathcal{O}(k)$, are adjacent either to $s$ but not to $t$, or to $t$ but not to $s$. As vertices from $C_{s}$ cannot be next to vertices from $C_{t}$ on $C$, they must come in large consecutive chunks along the cycle. To formalize this intuition, let us call a vertex in $C_{s}$ an internal s-vertex if both of its neighbors along the cycle are also from $C_{s}$, and internal $t$-vertices are defined analogously. We claim that except for $\mathcal{O}(k)$ vertices, all the vertices of $C$ are either internal $s$-vertices or internal $t$-vertices. Vertices from $C_{s}$ that are not internal $s$-vertices must have at least one neighbor along $C$ that is not from $C_{s}$ nor $C_{t}$, and the same holds for $C_{t}$. However, there are at most $14 k$ vertices in $V(C) \backslash C_{s} \backslash C_{t}$, and each of them can "spoil" at most two vertices of $C_{s}$ or $C_{t}$. Also note that a vertex of $C_{s t}$ must have vertices of $V(C) \backslash C_{s} \backslash C_{t}$ on both sides, as a vertex from $C_{s}$ cannot lie next to a vertex of $C_{t}$ on $C$. Thus the total number of internal $s$-vertices and internal $t$-vertices is at least

$$
\left(\left|C_{s}\right|-\left|C_{s t}\right|\right)+\left(\left|C_{t}\right|-\left|C_{s t}\right|\right)-2\left(\left|V(C) \backslash C_{s} \backslash C_{t}\right|-\left|C_{s t}\right|\right) \geq 2\left(\frac{n}{2}-3 k\right)-28 k=n-34 k .
$$

Now assume there is an edge between an internal $s$-vertex and an internal $t$-vertex. If this holds, the path $P_{i}$ can be inserted in $C$ in the same way as in the case of a single high-degree vertex above, see Figure 8 for an illustration. On the other hand, if there are no edges between internal $s$-vertices and internal $t$-vertices, then the graph induced on the sets of internal $s$-vertices and $t$-vertices is not connected, as these sets are both non-empty. Then removing at most $34 k$ vertices from $G$ leaves these sets disconnected. Thus we arrive to the terminal state (3) where we have a small separator. In order to apply Lemma 16 , it should contain $B$ as a subset, so after taking the union with $B$ its size is at most $35 k$.

\section{Dirac decomposition}

In this section, we define Dirac decompositions and show that, given a Dirac decomposition for a cycle in $G$, we can either find a longer cycle or solve the instance $(G, B, k)$ of Long DiRAC CyCLE in time single-exponential in $k+|B|$.

Definition 5 (Dirac decomposition and Dirac component). Let $G$ be a 2-connected graph, let $B$ be a subset of $V(G)$, and let $C$ be a cycle in $G$ of length at least $2 \delta(G-B)$. We say that two disjoint paths $P_{1}$ and $P_{2}$ in $G$ induce a Dirac decomposition for $C$ and $B$ in $G$ if

- The cycle $C$ is of the form $C=P_{1} P^{\prime} P_{2} P^{\prime \prime}$, where each of the paths $P^{\prime}$ and $P^{\prime \prime}$ has at least $\delta(G-B)-2$ edges. 


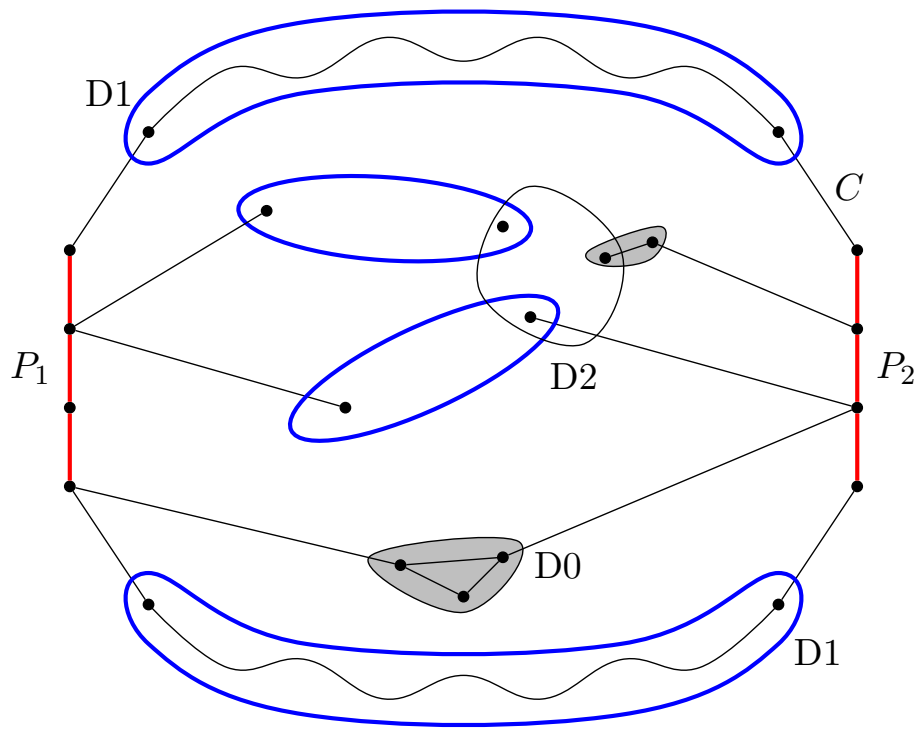

Figure 9: A schematic example of a Dirac decomposition, vertices belonging to $B$ are in light gray. Removing the paths $P_{1}$ and $P_{2}$ leaves two (D1)-type components that correspond to the long arcs $P^{\prime}$ and $P^{\prime \prime}$ of the starting cycle $C$, one (D2)-type component, and a component consisting only of vertices from $B$, denoted by D0. The four Dirac components are in thick blue.

- Let $G^{\prime}$ be the graph obtained from $G$ by applying $B$-refinement to every connected component $H$ of $G-V\left(P_{1} \cup P_{2}\right)$, except those components $H$ with $V(H) \subseteq B$. Note that no edges of the paths $P_{1}$ and $P_{2}$ are contracted. Then for every connected component $H^{\prime}$ of $G^{\prime}-V\left(P_{1} \cup P_{2}\right)$, except those with $V\left(H^{\prime}\right) \subseteq B$, holds $\left|V\left(H^{\prime}\right)\right| \geq 3$ and one of the following.

(D1) $H^{\prime}$ is 2-connected and the maximum size of a matching in $G^{\prime}$ between $V\left(H^{\prime}\right)$ and $V\left(P_{1}\right)$ is one, and between $V\left(H^{\prime}\right)$ and $V\left(P_{2}\right)$ is also one;

(D2) $H^{\prime}$ is not 2-connected, exactly one vertex of $P_{1}$ has neighbors in $H^{\prime}$, that is, $\mid N_{G^{\prime}}\left(V\left(H^{\prime}\right)\right) \cap$ $V\left(P_{1}\right) \mid=1$, and no inner vertex from a leaf-block of $H^{\prime}$ has a neighbor in $P_{2}$;

(D3) The same as (D2), but with $P_{1}$ and $P_{2}$ interchanged. That is, $H^{\prime}$ is not 2-connected, $\left|N_{G^{\prime}}\left(V\left(H^{\prime}\right)\right) \cap V\left(P_{2}\right)\right|=1$, and no inner vertex from a leaf-block of $H^{\prime}$ has a neighbor in $P_{1}$.

- There is exactly one connected component $H$ in $G-V\left(P_{1} \cup P_{2}\right)$ with $V(H) \backslash B=V\left(P^{\prime}\right) \backslash(B \cup$ $\left.\left\{s^{\prime}, t^{\prime}\right\}\right)$, where $s^{\prime}$ and $t^{\prime}$ are the endpoints of $P^{\prime}$. Analogously, there is exactly one connected component $H$ in $G-V\left(P_{1} \cup P_{2}\right)$ with $V(H) \backslash B=V\left(P^{\prime \prime}\right) \backslash\left(B \cup\left\{s^{\prime \prime}, t^{\prime \prime}\right\}\right)$.

The set of Dirac components for a Dirac decomposition is defined as follows. First, for each component $H^{\prime}$ of type (D1), $H^{\prime}$ is a Dirac component of the Dirac decomposition. Second, for each leaf-block of each $H^{\prime}$ of type (D2), or of type (D3), this leaf-block is also a Dirac component of the Dirac decomposition. For an example of a Dirac decomposition, see Figure 9.

Note that Lemma 5 holds for an arbitrary cycle $C$ if we replace Erdös-Gallai components and Erdős-Gallai decompositions with Dirac components and Dirac decompositions. We give the analogue of this lemma below without proof, since it is identical to the proof of Lemma 5 . 
Lemma 17. Let $G$ be a 2-connected graph, $B \subseteq V(G), C$ be a cycle in $G$. Let paths $P_{1}, P_{2}$ induce a Dirac decomposition for $C$ and $B$ in $G$. Let $M$ be a Dirac component of the Dirac decomposition and $P$ be a path in $G$ such that $P$ contains at least one vertex in $V\left(P_{1}\right) \cup V\left(P_{2}\right)$. If $P$ enters $M$, then all vertices of $M$ hit by $P$ appear consecutively on $P$.

We now want to prove an analogue of Lemma 7 showing that if a long cycle in $G$ exists, then it suffices to look for a long cycle entering a Dirac component. For that we first require the following weaker result.

Lemma 18. Let $G$ be a 2-connected graph, $B \subseteq V(G), C$ be a cycle in $G$ of length less than $2 \delta(G-B)+k$. Let paths $P_{1}, P_{2}$ induce a Dirac decomposition for $C$ and $B$ in $G$. If $H^{\prime}$ is a (D2)-type or a (D3)-type component of the Dirac decomposition and $S$ is a B-leaf-block separator of $H^{\prime}$, then there is a cycle of length at least $\frac{1}{2}(5 \delta(G-B)-|S|-(k+5))$ that enters a Dirac component in $G$.

Proof. Without loss of generality, let $H^{\prime}$ be a (D2)-type component of the Dirac decomposition. Take a vertex $v \in V\left(H^{\prime}\right)$ that is not an inner vertex of a leaf-block of $H^{\prime}$ and has a neighbour in $P_{2}$. Such vertex always exists by definition of a Dirac decomposition.

Let $S$ be a $B$-leaf-block separator of $H^{\prime}$. By Lemma 6, there is a $(c, v)$-path of length at least $\frac{1}{2} \delta\left(H^{\prime}-B\right)-\frac{1}{2}|S|$ in $H^{\prime}$ for some cut-vertex $c$ of a leaf-block of $H^{\prime}$. Also $\delta\left(H^{\prime}-B\right) \geq$ $\delta(G-B)-\left|V\left(P_{1}\right) \cup V\left(P_{2}\right)\right| \geq \delta(G-B)-(k+5)$, since the total length of $P_{1}$ and $P_{2}$ is at most $k+3$. Denote this leaf-block of $H^{\prime}$ by $L$.

Note that $\delta(L-(B \cup\{c\})) \geq \delta(G-B)-2$ by properties of (D2)-type components. By Corollary3. there is a path of length at least $\delta(G-B)-2$ between $c$ and any other vertex in $L$. Let $u$ be an inner vertex in $L$ that has a neighbour in $P_{1}$.

Combine the $(u, c)$-path inside $L$ with the $(c, v)$-path going outside $L$ in $H^{\prime}$. The obtained path is a $(u, v)$-path of length at least $(\delta(G-B)-2)+\left(\frac{1}{2} \delta\left(H^{\prime}-B\right)-\frac{1}{2}|S|\right)$.

Since $u$ and $v$ have neighbours in $V\left(P_{1}\right)$ and $V\left(P_{2}\right)$ respectively, we obtain a chord of $C$ of length at least $\delta(G-B)+\frac{1}{2} \delta\left(H^{\prime}-B\right)-\frac{1}{2}|S|$. The chord splits $C$ into two arcs, one of which is of length at least $\delta(G-B)$. Combine this arc with the chord and obtain a cycle of length at least $2 \delta(G-B)+\frac{1}{2} \delta\left(H^{\prime}-B\right)-\frac{1}{2}|S| \geq \frac{5}{2} \delta(G-B)-\frac{1}{2}|S|-\frac{1}{2}(k+5)$.

The following lemma is an analogue of Lemma 7 for Dirac components. In contrast to Lemma 17 , the proof is significantly different from the proof of Lemma 7 .

Lemma 19. Let $G$ be a graph, $B \subseteq V(G)$ be a subset of its vertices and $P_{1}, P_{2}$ induce a Dirac decomposition for a cycle $C$ of length less than $2 \delta(G-B)+k$ in $G$. Let $k$ be an integer such that $6 k+4|B|+6<\delta(G-B)$. If there exists a cycle of length at least $2 \delta(G-B)+k$ in $G$ that contains at least one vertex in $V\left(P_{1}\right) \cup V\left(P_{2}\right)$, then there exists a cycle of length at least $2 \delta(G-B)+k$ in $G$ that enters a Dirac component.

Proof. Suppose that there exists a cycle $C^{\prime}$ of length at least $2 \delta(G-B)+k$ in $G$ that contains at least one vertex in $V\left(P_{1}\right) \cup V\left(P_{2}\right)$. If $C^{\prime}$ already contains an edge of a Dirac component, we are done. We now assume that $C^{\prime}$ does not contain any edge of any Dirac component. We show how to use $C^{\prime}$ and construct a cycle of length at least $2 \delta(G-B)+k$ in $G$ that contains an edge of a Dirac component of the given Dirac decomposition.

Let $W$ be the set of all vertices of $G$ that are vertices of non-leaf-blocks of (D2)-type or (D3)-type components in the Dirac decomposition. We start with the following claim.

Claim 12. $\left|W \cap V\left(C^{\prime}\right)\right|>5 k$. 
Proof of Claim 12. This is a counting argument. Note that $C^{\prime}$ cannot contain an edge with both endpoints inside a Dirac component of $G$. Since Dirac components of $G$ are (D1)-type components of the Dirac decomposition and leaf-blocks of (D2)-type or (D3)-type connected components, each edge of $C^{\prime}$ has an endpoint either in $V\left(P_{1}\right) \cup V\left(P_{2}\right) \cup B$, or inside a non-leaf-block of a (D2)-type or a (D3)-type connected component. The union of the vertex sets of the non-leaf-blocks form the set $W$. Hence, $\left(W \cap V\left(C^{\prime}\right)\right) \cup V\left(P_{1}\right) \cup V\left(P_{2}\right) \cup B$ is a vertex cover of $C^{\prime}$.

Note that a vertex cover of any cycle consists of at least half of its vertices. Then

$$
2\left|\left(W \cap V\left(C^{\prime}\right)\right) \cup V\left(P_{1}\right) \cup V\left(P_{2}\right) \cup B\right| \geq\left|V\left(C^{\prime}\right)\right| \geq 2 \delta(G-B)+k .
$$

Immediately we get that

$$
2\left|W \cap V\left(C^{\prime}\right)\right| \geq 2 \delta(G-B)+k-2\left|V\left(P_{1}\right) \cup V\left(P_{2}\right)\right|-2|B| \geq 2 \delta(G-B)+k-2(k-2)-2|B|>10 k .
$$

The following claim is useful for constructing long chords of $C^{\prime}$ going through the Dirac components that are leaf-blocks.

Claim 13. Let $H^{\prime}$ be a (D2)-type or a (D3)-type component in the Dirac decomposition. $C^{\prime}$ does not contain any inner vertex of the leaf-blocks of $H^{\prime}$.

Proof of Claim 13. Suppose that $C^{\prime}$ contains some vertex $u \in V\left(H^{\prime}\right)$ that is an inner vertex of some leaf-block $L$ of $H^{\prime}$. As $L$ is a Dirac component of $G, C^{\prime}$ cannot contain any edge of $L$, so $C^{\prime}$ should enter $L$ from $V\left(P_{1}\right) \cup V\left(P_{2}\right)$ through $u$ and leave it immediately. By definition of Dirac decompositions, the only option to enter or leave $L$ is to go through the only vertex in $V\left(P_{1}\right)$ (if $H^{\prime}$ is of type (D2) or in $V\left(P_{2}\right)$ (if $H^{\prime}$ is of type (D3). As $C^{\prime}$ cannot contain any vertex twice, this is not possible.

We now use the above claims to construct either a family of long chords of $C^{\prime}$ going through Dirac components, or a $B$-leaf-block separator of some of the (D2)-type or (D3)-type components in the Dirac decomposition.

To construct the first chord of $C^{\prime}$, take a vertex $w_{1} \in W$. Since $w_{1}$ is a vertex of a separable component $H^{\prime}$, there is a cut vertex $c_{1}$ of a leaf-block $L_{1}$ of $H^{\prime}$ reachable from $w_{1}$ inside $H^{\prime}$. The leaf-block $L_{1}$ contains also at least one vertex $v_{1} \neq c_{1}$ that is connected to $V\left(P_{1}\right)$ (if $H^{\prime}$ is of type (D2) or to $V\left(P_{2}\right)$ (if $H^{\prime}$ is of type (D3) outside $H^{\prime}$. We know that $\delta\left(L_{1}-\left(B \cup\left\{c_{1}\right\}\right)\right) \geq$ $\delta\left(G-\left(B \cup\left\{c_{1}\right\}\right)\right)-1 \geq \delta(G-B)-2$, since the only outside neighbour of vertices in $L_{1}-\left(B \cup c_{1}\right)$, apart from vertices in $B$, is a single vertex in $V\left(P_{1}\right)$ or $V\left(P_{2}\right)$. By Corollary 3 , there exists an $\left(c_{1}, v_{1}\right)$-path inside $L_{1}$ of length at least $\delta(G-B)-2$. Combine this with $\left(w_{1}, c_{1}\right)$-path inside $H^{\prime}$ and obtain a $\left(w_{1}, v_{1}\right)$-path inside $H^{\prime}$.

Note that the constructed $\left(w_{1}, v_{1}\right)$-path can contain vertices from $W$ apart from $w_{1}$. Let $w_{1}^{\prime} \in W$ be the vertex on the $\left(w_{1}, v_{1}\right)$-path farthest from $w_{1}$. Note that the $\left(w_{1}^{\prime}, v_{1}\right)$-subpath does not contain any vertex from $W$ except $w_{1}^{\prime}$, and it still contains the $\left(c_{1}, v_{1}\right)$-path as a subpath by Claim 13 . Hence, we obtain a $\left(w_{1}^{\prime}, v_{1}\right)$-path of length at least $\delta(G-B)-2$ inside $H^{\prime}$ that does not contain any vertex in $W \backslash\left\{w_{1}^{\prime}\right\}$. To obtain a long chord of $C^{\prime}$, it is left to reach the vertex in $V\left(P_{1}\right) \cup V\left(P_{2}\right)$ from $v_{1}$ outside $H^{\prime}$, and then follow the cycle $C$ until a vertex $v_{1}^{\prime}$ of $C^{\prime}$ is reached. This is always possible since $V(C) \cap V\left(C^{\prime}\right) \supseteq\left(V\left(P_{1}\right) \cup V\left(P_{2}\right)\right) \cap V\left(C^{\prime}\right) \neq \emptyset$. We obtain a chord of length at least $\delta(G-B)-1$ connecting $w_{1}^{\prime}$ and $v_{1}^{\prime}$.

To construct the second chord, we follow the same process for a vertex $w_{2} \in W \backslash\left\{w_{1}^{\prime}\right\}$. When constructing the path going from $w_{2}$ to a cut vertex of a leaf-block, we prohibit this path from going 
through $w_{1}^{\prime}$. If $w_{1}^{\prime}$ separates $w_{2}$ from all leaf-block cut vertices, then we obtain a small $B$-leaf-block separator of $H^{\prime}$. Otherwise, we obtain a $\left(w_{2}^{\prime}, v_{2}^{\prime}\right)$-chord of $C^{\prime}$ of length at least $\delta(G-B)-1$ that does not contain any vertex in $W \backslash\left\{w_{1}^{\prime}, w_{2}^{\prime}\right\}$. It is important that during the construction of different chords we always follow $C$ in the same direction.

Repeat this process $3 k$ times and obtain either a family of $3 k\left(w_{i}^{\prime}, v_{i}^{\prime}\right)$-chords of $C^{\prime}$ of length at least $\delta(G-B)-1$, or a $B$-leaf-block separator of size at most $3 k$. If it is the latter case, then, by Lemma 18 , there is a cycle of length at least $2 \delta(G-B)+\frac{1}{2}(\delta(G-B)-3 k-(k+5))>2 \delta(G-B)+k$ that enters a Dirac component. We now assume that a family of chords is obtained. The following claim is useful.

Claim 14. If for some $i, j \in[3 k]$ we have $v_{i}^{\prime} \neq v_{j}^{\prime}$, then the chords between $w_{i}^{\prime}$ and $v_{i}^{\prime}$ and between $w_{j}^{\prime}$ and $v_{j}^{\prime}$ do not have any common vertex.

Proof of Claim 14. Note that $v_{i}^{\prime}$ or $v_{j}^{\prime}$ depend only on the vertex in $V\left(P_{1}\right)$ or in $V\left(P_{2}\right)$ which we start following $C$ from. If $v_{i}^{\prime} \neq v_{j}^{\prime}$, then they were found when starting from different vertices. Then the $i^{\text {th }}$ and the $j^{\text {th }}$ chords were constructed from different components of the Dirac decomposition, as for each separable component there is only one vertex in $V\left(P_{1}\right) \cup V\left(P_{2}\right)$ that is adjacent to inner vertices of leaf-blocks of this component. Therefore, the $\left(w_{i}^{\prime}, v_{i}\right)$ - and $\left(w_{j}^{\prime}, v_{j}\right)$-subpaths of the chords do not have common vertices. The $\left(v_{i}, v_{i}^{\prime}\right)$ - and $\left(v_{j}, v_{j}^{\prime}\right)$-subpaths also cannot have any common vertex as $v_{i}^{\prime}$ and $v_{j}^{\prime}$ were found as the first vertices from $V\left(C^{\prime}\right)$ on $C$ when following $C$ in the same direction.

Definition 6. Consider two chords of $C$ that do not have common endpoints. Denote the endpoints of one chord by $s$ and $t$ and of the other by $p$ and $q$. We say that these two chords of $C$ intersect graphically, if the vertices $s, t, p$ and $q$ are located in the order $s, p, t, q$ on $C$ when following $C$ in one or the other direction. In other words, two chords intersect graphically if each chord cuts $C$ into two arcs each containing exactly one endpoint of the other chord.

We can now show that if two chords in the constructed family intersect graphically, then we can find a long cycle that contains these two chords. Assume that there are $i, j \in[3 k]$ such that $v_{i}^{\prime} \neq v_{j}^{\prime}$ and the vertices $w_{i}^{\prime}, v_{i}^{\prime}, w_{j}^{\prime}, v_{j}^{\prime}$ are located in the order $w_{i}^{\prime}, w_{j}^{\prime}, v_{i}^{\prime}, v_{j}^{\prime}$ on $C^{\prime}$ when following $C^{\prime}$ in one of the two directions. These four vertices split $C^{\prime}$ into four arcs. A pair of opposite arcs together with the two chords constitute a cycle in $G$. Take the pair of arcs with the longest total length. This total length is at least $\frac{1}{2}\left|V\left(C^{\prime}\right)\right| \geq \delta(G-B)+\frac{k}{2}$. Combining these two arcs with the two chords, we obtain a cycle of length at least $3 \delta(G-B)+\frac{k}{2}-2>2 \delta(G-B)+k$. This cycle enters two Dirac components, as each chord enters a Dirac component. Hence, in this case the desired cycle exists and the lemma is proved.

We now assume that no two chords of the family intersect graphically. In this case we can arrange them in an order from left to right. That is, we can choose a permutation $\pi \in S_{3 k}$ and for each $i \in[3 k]$ either a pair $a_{i}=w_{\pi_{i}}^{\prime}$ and $b_{i}=v_{\pi_{i}}^{\prime}$ or a pair $a_{i}=v_{\pi_{i}}^{\prime}$ and $b_{i}=w_{\pi_{i}}^{\prime}$ in such a way that following the cycle $C^{\prime}$ starting from $a_{1}$ one will read the $6 k$ vertices in the order $a_{1}, a_{2}, \ldots, a_{3 k}, b_{3 k}, b_{3 k-1}, \ldots, b_{1}$ (see Figure 10). It is important to note that $a_{i}=a_{i+1}$ or $b_{i}=b_{i+1}$ might hold true for any $i \in[3 k-1]$, but at least one of $a_{i} \neq a_{i+1}$ and $b_{i} \neq b_{i+1}$ always holds.

Take an arbitrary $i \in[3 k]$. The chord between $a_{i}$ and $b_{i}$ splits $C^{\prime}$ into two arcs. We call them left arc of $\left(a_{i}, b_{i}\right)$, that is, the arc that contains vertices $a_{1}, b_{1}, a_{2}, b_{2}, \ldots, a_{i}, b_{i}$ and the right arc of $\left(a_{i}, b_{i}\right)$, that is, the arc that contains vertices $a_{i}, b_{i}, a_{i+1}, b_{i+1}, \ldots, a_{3 k}, b_{3 k}$. If at least one of these arcs has length at least $\delta(G-B)+k+1$, we call the chord between $a_{i}$ and $b_{i}$ a good chord. Then the chord together with the longer arc constitute a cycle of length at least $2 \delta(G-B)+k$. This 


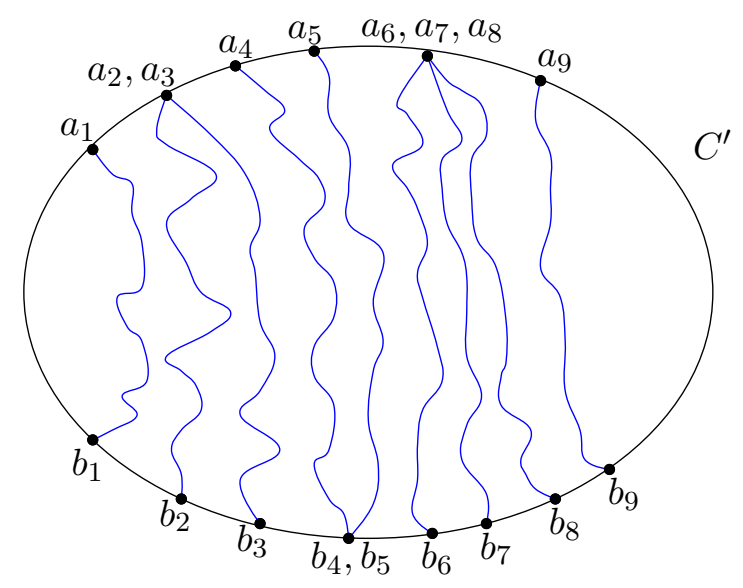

Figure 10: The family of long chords of the cycle $C^{\prime}$ that do not intersect graphically pairwise.

cycle enters a Dirac component, so the proof is complete if there is a good chord in the constructed family of chords. We now show that there exists at least one good chord in the family.

If the chord between $a_{1}$ and $b_{1}$ is good, then we are done. Otherwise, both arcs of $\left(a_{1}, b_{1}\right)$ are of length at most $\delta(G-B)+k$. Since the length of $C^{\prime}$ is at least $2 \delta(G-B)+k$, it follows that the length of both these arcs is at least $\delta(G-B)$. Consider the chord between $a_{2}$ and $b_{2}$. Note that the left arc of $\left(a_{2}, b_{2}\right)$ is longer than the left arc of $\left(a_{1}, b_{1}\right)$ because $\left(a_{1}, b_{1}\right) \neq\left(a_{2}, b_{2}\right)$. Hence, the length of the left arc of $\left(a_{2}, b_{2}\right)$ is at least $\delta(G-B)+1$. Analogously, we can show that for each $i \in[3 k]$ the length of the left arc of $\left(a_{i}, b_{i}\right)$ is at least $\delta(G-B)+i-1$. Hence, for any $j \in[2 k]$ the chord between $a_{k+j}$ and $b_{k+j}$ is a good chord. The proof of the lemma is complete.

Finally, we state and prove the main theorem of this section.

Theorem 16. Let $(G, B, k)$ be a given instance of LONG DIRAC CYCLE. There is an algorithm that, given a cycle $C$ in $G$ and two paths $P_{1}, P_{2}$ that induce an Dirac decomposition for $C$ and $B$ in $G$, in time $2^{\mathcal{O}(k+|B|)} \cdot n^{\mathcal{O}(1)}$ either

- Solves $(G, B, k)$, or

- Finds a cycle longer than $C$ in $G$.

Proof. The algorithm considers several cases. If the given cycle $C$ is of length at least $2 \delta(G-B)+k$, then the algorithm correctly determines that $(G, B, k)$ is a yes-instance. Hence, we assume that $|V(C)|<2 \delta(G)+k$. From now on, we also assume that $6 k+4|B|+6<\delta(G-B)$, otherwise the algorithm solves $(G, B, k)$ using the algorithm from Proposition 12 .

Suppose now that $G$ contains a cycle $C^{\prime}$ of length at least $2 \delta(G-B)+k$. We show how the algorithm finds some cycle of length at least $2 \delta(G-B)+k$ in $G$ or enlarges $C$ provided that $C^{\prime}$ exists. We are now interested in the set $X=V\left(C^{\prime}\right) \cap\left(V\left(P_{1}\right) \cup V\left(P_{2}\right)\right)$. Depending on its cardinality, there are several cases. In most of the cases, it is possible for the algorithm to replace one arc of $C$ with a longer arc that is found using the algorithm for LONG ERDös-GALLAI $(s, t)$-PATH given by Theorem 5. The algorithm is usually applied to a component in $G-V\left(P_{1} \cup P_{2}\right)$ with the goal of finding a path of length at least $\delta(G-B)+k / 2$. Since $\delta\left(G-\left(V\left(P_{1} \cup P_{2}\right) \cup B\right)\right)>\delta(G-B)-k-4$, the running time of the algorithm is still bounded by $2^{\mathcal{O}(k+|B|)} \cdot n^{\mathcal{O}(1)}$.

Case 1: $|X|=0$. Then $C^{\prime}$ is completely contained in some connected component $H$ of $G-V\left(P_{1} \cup\right.$ $\left.P_{2}\right)$. This component cannot be contained in $B$, since $|B|<\delta(G-B)$. So after the $B$-refinements, 
this component is a component $H^{\prime}$ of type (D1), (D2) or (D3), Note that only the leaf-blocks that have all inner vertices in $B$ are contracted in $H$ to obtain $H^{\prime}$. The cycle $C^{\prime}$ does not pass through cut vertices. Moreover, its length is greater than $B$. Hence, no edge of $C^{\prime}$ is contracted during the $B$-refinements of $H$, so $C^{\prime}$ is fully contained in $H^{\prime}$.

By the last property of Dirac decompositions, there are exactly two connected components in $G-\left(V\left(P_{1}\right) \cup V\left(P_{2}\right)\right)$ containing vertices of $C$. Both of them contain at most $\delta(G-B)+k+|B|$ vertices, as the length of $C$ is less than $2 \delta(G-B)+k$. Hence, $H^{\prime}$ does not share any vertices with the initial cycle $C$, as $\left|V\left(H^{\prime}\right)\right| \geq\left|V\left(C^{\prime}\right)\right| \geq 2 \delta(G-B)+k$.

If $H^{\prime}$ is of type (D1), then it contains a path of length at least $\left|V\left(C^{\prime}\right)\right| / 2$ between any pair of vertices by Claim 9. Take any pair $(s, t)$ of neighbours of $H^{\prime}$ in $V\left(P_{1}\right)$ and $V\left(P_{2}\right)$ respectively. There is an $(s, t)$-path in $G$ of length at least $\left|V\left(C^{\prime}\right)\right| / 2+2>\delta(G-B)+k / 2$ that contains only vertices in $V\left(H^{\prime}\right) \cup B$ as internal vertices. One of the arcs of $C$ between $s$ and $t$ have length less than $\delta(G-B)+k / 2$, so it can be replaced with the obtained $(s, t)$-path, making $C$ longer.

If $H^{\prime}$ is of type (D2), then it is not 2-connected. Denote the only neighbour of $H^{\prime}$ in $V\left(P_{1}\right)$ by $s$. Note that the graph $G^{\prime}\left[V\left(H^{\prime}\right) \cup\{s\}\right]$ is 2-connected and still contains the cycle $C^{\prime}$. Hence, it contains a path of length at least $\left|V\left(C^{\prime}\right)\right| / 2$ between any pair of vertices. Now take any neighbour of $H^{\prime}$ in $V\left(P_{2}\right)$, say $t$. It is easy to obtain an $(s, t)$-path in $G$ of length at least $\left|V\left(C^{\prime}\right)\right| / 2+1$ going only through vertices in $V\left(H^{\prime}\right) \cup B$. Again, this path is a replacement for one of the arcs between $s$ and $t$ in $G$. The case of type (D3) is symmetrical.

Conclusion of Case 1. To handle this case, the algorithm unconditionally iterates over all components in $G-V\left(P_{1} \cup P_{2}\right)$ and tries to find a suitable path of length at least $\delta(G-B)+k / 2$ in a 2-connected subgraph of $G$ using the algorithm of Theorem 5 for Long ERDős-GallaI $(s, t)$ PATH. Note that a subgraph picked by the algorithm is always a graph $H^{\prime}$ with $\delta\left(H^{\prime}-B^{\prime}\right) \geq$ $\delta\left(G-B^{\prime}-\left(V\left(P_{1}\right) \cup V\left(P_{2}\right)\right)\right.$, where $\left|B^{\prime}\right| \leq|B|+1$. Hence, $\delta\left(H^{\prime}-B^{\prime}\right) \geq \delta(G-B)-(k+5)$, so the algorithm for LONG ERDŐS-GALLAI $(s, t)$-PATH always runs in $2^{\mathcal{O}(k+|B|)} \cdot n^{\mathcal{O}(1)}$ running time. If a suitable path is found, $C$ is made longer by the algorithm, and the algorithm outputs the longer cycle and terminates. Otherwise, there are no long cycles $C^{\prime}$ with $|X|=0$ in $G$.

Case 2. $|X|=1$. Denote the only vertex in $X$ by $v$. Note that $C^{\prime}$ passes through only one connected component in $G-V\left(P_{1} \cup P_{2}\right)$, since $C^{\prime}-v$ is a path having no common vertices with $P_{1}$ or $P_{2}$. Denote the component containing $C^{\prime}-v$ in $G-V\left(P_{1} \cup P_{2}\right)$ by $H$. We know that $H$ consists of at least $2 \delta(G-B)+k-1$ vertices, so it is not fully contained in $B$ and does not share any vertex with the initial cycle $C$, just as in the previous case.

Without loss of generality, assume that $v \in V\left(P_{1}\right)$. Denote by $H^{\prime}$ the connected component $H$ after the $B$-refinements. Independently of the type of $H^{\prime}$ in the Dirac decomposition, there is a vertex in $H$ with a neighbour in $V\left(P_{2}\right)$. Hence, there is a path starting in a certain vertex $u \in V\left(P_{2}\right)$ and going to a certain vertex $z \in V\left(C^{\prime}-v\right)$ through $H$ in $G$. Take the longer arc between $z$ and $v$ on $C^{\prime}$ and combine it with the path between $u$ and $z$. The obtained path is of length at least $\left|V\left(C^{\prime}\right)\right| / 2+1$ and is a replacement for the shorter arc between $u$ and $v$ on $C$.

The only obstacle here is that $H$ is not necessarily 2-connected. However, the graph $G[V(H) \cup$ $\{v\}]$ still contains the whole cycle $C^{\prime}$. If $H^{\prime}$ is of type (D1), then $G[V(H) \cup\{v\}]$ is necessarily 2connected after $B$-refinements. If $H^{\prime}$ is of type $(D 2)$, then $G[V(H) \cup\{v\}]$ also becomes 2-connected after $B$-refinements, as $v$ is the only neighbour in $V\left(P_{1}\right)$ connecting all leaf-blocks of $H^{\prime}$ together. In either of the two cases, if $z$ is fixed, the path between $v$ and $z$ can be found using the algorithm for LONG ERDős-Gallai $(s, t)$-PATH.

Finally, if $H^{\prime}$ is of type(D3), then $u$ is the only neighbour of $H^{\prime}$ in $V\left(P_{2}\right)$ after the $B$-refinements. Then the graph $G[V(H) \cup\{u, v\}]$ is necessarily 2-connected after $B$-refinements and the desired $(u, v)$-path can be found inside it. 
Conclusion of Case 2. The algorithm iterates over all suitable pairs of $v$ and $H$, and iterates over all possible options of $z$ or $u$ when necessary. When this triple is fixed, it is left to apply the algorithm of Theorem 5 to the corresponding $B$-refinement as described above. Again, in time $2^{\mathcal{O}(k+|B|)} \cdot n^{\mathcal{O}(1)}$ our algorithm either makes the initial cycle $C$ longer and stops or correctly determines that no long cycle $C^{\prime}$ with $|X|=1$ exists.

Case 3: $|X|=2$. Let $X=\{s, t\}$. Starting from this case, we need to consider Dirac components that $C^{\prime}$ enters. By Lemma 19, we can assume that $C^{\prime}$ enters some Dirac component $M$. The cycle $C^{\prime}$ has two arcs between $s$ and $t$. At least one of them enters $M$ and, by Lemma 17, we know that all vertices of $M$ appear consecutively on this arc.

Suppose that both arcs between $s$ and $t$ enter $M$. If both $s, t \in V\left(P_{1}\right)$ or $s, t \in V\left(P_{2}\right)$, then we obtain a matching of size two between $V\left(P_{i}\right)$ and a Dirac component, which is not possible by the definition of Dirac decomposition. Hence, we can assume that $s \in V\left(P_{1}\right)$ and $t \in V\left(P_{2}\right)$. Since both arcs enter $M$, there is a connected component $H$ in $G-V\left(P_{1} \cup P_{2}\right)$ that contains $M$ and both arcs of $C^{\prime}$. Thus $C^{\prime}$ is contained in $G[V(H) \cup\{s, t\}]$. After the $B$-refinements, $G[V(H) \cup\{s, t\}]$ also contains the whole cycle $C^{\prime}$ similarly to the arguments above. Note that after the $B$-refinements this graph is 2-connected, since if $H^{\prime}$ is of type (D2) or of type (D3), the vertex $s$ or the vertex $t$ correspondingly is the vertex connecting all its leaf-blocks together. Hence, the algorithm can look for an $(s, t)$-path of length at least $\delta(G-B)+k / 2$ inside the graph $G[V(H) \cup\{s, t\}]$ with applied $B$-refinements. The component $H$ contains at least $2 \delta(G-B)+k-2$ vertices, so it does not share vertices with $C$. Thus, this $(s, t)$-path is a suitable replacement for a shorter arc between $s$ and $t$ on $C$.

Suppose now that only one arc of $C^{\prime}$ between $s$ and $t$ enters $M$. Then, by Lemma 17, we can be sure that all vertices of $M$ appear consecutively on $C^{\prime}$. That is, there are two vertices $u, v \in V(M) \cap V\left(C^{\prime}\right)$ such that one of the arcs of $C^{\prime}$ between $u$ and $v$ is a $(u, v)$-path inside $M$, and the other arc is a $(u, v)$-path in $G$ that does not contain any vertex of $M$ as internal vertex. In this case, the algorithm can find these two arcs in the following way.

When $s, t$ and $M$ are fixed, the algorithm iterates over all pairs of distinct vertices $u, v \in V(M)$. Firstly, the algorithm tries to find a path between $u$ and $v$ outside $M$. Since there is a path of length at least $\delta(G-B)-2$ between any pair of vertices in $M$, the outer path length $\delta(G-B)+k+2$ is sufficient to construct a cycle of length $2 \delta(G-B)+k$ in $G$. Hence, to find the $(u, v)$-path outside $M$, the algorithm removes all vertices in $V(M) \backslash\{u, v\}$ from $G$, and adds a single edge between $u$ and $v$ in $G$. Note that if the outer path between $u$ and $v$ exists, then $G$ remains 2 -connected after these operations, since $u$ and $v$ still belong to the same cycle. If $G$ is not 2 connected, then the choice of $u$ and $v$ was wrong. Otherwise, we apply the algorithm of Theorem 5 to the changed graph $G$ to find a long path between $u$ and $v$. If a $(u, v)$-path of length at least $\delta(G-B)+k+2$ exists, then $(G, B, k)$ is a yes-instance. Otherwise, the algorithm finds the longest path between $u$ and $v$. This is done in $2^{\mathcal{O}(k+|B|)} \cdot n^{\mathcal{O}(1)}$ time. Note that a $(u, v)$-path of length at least $\delta\left(G-\left(V\left(P_{1}\right) \cup V\left(P_{2}\right) \cup B \cup\{u, v\}\right)\right) \geq \delta(G-B)-k-6$ always exists in the modified graph $G$ by Corollary 3 .

If the outer $(u, v)$-path is found, it is left for us to find a long path between $u$ and $v$ inside $M$. If this path is of length at least $\delta(G-B)+2 k+6$, then $(G, B, k)$ is a yes-instance of LONG DiraC CyCle, since the outer $(u, v)$-path is of length at least $\delta(G-B)-k-6$. Thus, using the algorithm for LONG ERDős-GAllai $(s, t)$-PATH, we either find a sufficiently long path between $u$ and $v$ inside $M$, such that the total length of this path and the outer path is at least $2 \delta(G-B)+k$, or conclude that none exists and move on to the next choice of $u$ and $v$.

Conclusion of Case 3. To handle this case, the algorithm iterates over all possible pairs of $s$ and $t$. To handle the case when $C^{\prime}$ enters just one connected component of $G-V\left(P_{1} \cup\right.$ 
$P_{2}$ ), the algorithm behaves similarly to previous cases. Additionally, to handle the case when $C^{\prime}$ contains a consecutive path inside a Dirac component, the algorithm iterates over all possible Dirac components $M$, and pairs $u, v \in V(M)$ and tries to construct a long cycle using two calls to the algorithm for LONG ERDős-Gallai $(s, t)$-PATH.

Case 4. $|X| \geq 3$. Then $X$ contains three distinct vertices $v_{1}, v_{2}, v_{3}$. These vertices split $C^{\prime}$ into three arcs $A_{1}, A_{2}, A_{3}$, where $A_{1}$ is the arc between $v_{1}$ and $v_{2}$ that does not contain $v_{3}, A_{2}$ is the arc between $v_{2}$ and $v_{3}$ that does not contain $v_{1}$, and $A_{3}$ is the arc between $v_{3}$ and $v_{1}$ that does not contain $v_{2}$. By Lemma 19, we can assume that $C^{\prime}$ enters a Dirac component $M$. Without loss of generality, assume that $A_{1}$ enters $M$. By Lemma 17, all vertices of $M$ appear consecutively on this arc.

Claim 15. $A_{2}$ and $A_{3}$ do not contain any vertex of $M$.

Proof of Claim 15. Take the arc between $v_{1}$ and $v_{3}$ that contains $v_{2}$, i.e. the union of $A_{1}$ and $A_{2}$. We now that this arc enters $M$, so by Lemma 17 all vertices of $M$ appear consecutively on it. But $A_{1}$ contains at least two vertices of $M$. Hence, $A_{2}$ cannot contain any vertex of $M$, as $v_{2} \notin V(M)$ separates $A_{1}$ and $A_{2}$ on the arc between $v_{1}$ and $v_{3}$.

To show that $A_{3}$ does not contain any vertex of $M$, take the arc between $v_{3}$ and $v_{2}$ that contains $v_{1}$, i.e. the union of $A_{3}$ and $A_{1}$. Again, by Lemma 17 this arc contains vertices of $M$ consecutively, but $v_{1}$ divides $A_{3}$ and $A_{1}$ on the arc. Since $A_{1}$ contains at least two vertices of $M, A_{3}$ cannot contain any of them.

The claim shows that vertices of $M$ induce an arc of $C^{\prime}$, similarly to the second part of Case 3 . Hence, this case can be handled by the algorithm in exactly the same way as in Case 3 .

Conclusion of Case 4. To cover this case, our algorithm first fixes $v_{1}, v_{2} \in V\left(P_{1} \cup P_{2}\right)$. Then it iterates over all Dirac components of the Dirac decomposition and tries to combine a long cycle from two paths, one inside the Dirac component, and one outside. This is done in exactly the same way as in the second part of Case 3 .

The list of cases is exhaustive, so if $C^{\prime}$ exists, our algorithm enlarges the initial cycle $C$ or finds a cycle of length at least $2 \delta(G-B)+k$ in $G$, determining that $(G, B, k)$ is a yes-instance. If $C^{\prime}$ does not exist, the algorithm does not find any long arc or long cycle in $G$, and safely decides that $(G, B, k)$ is a no-instance. This concludes the proof.

\section{Long Dirac Cycle: Putting all together}

In this section we finalize the proof of Theorem 3 by combining the main results of previous sections. This relies crucially on the following lemma. The most important part of this lemma is the construction of a Dirac decomposition.

Lemma 20. Let $G$ be an n-vertex 2-connected graph, $B \subseteq V(G)$, and $k$ be an integer such that $0<k \leq \frac{1}{24} \delta(G-B)$, and

$$
2 k+2|B|+12 \leq \delta(G-B)<\frac{n}{2}-\frac{|B|+k}{2} .
$$

Then there is an algorithm that, given a cycle $C$ of length less than $2 \delta(G-B)+k$ in polynomial time finds either

- Longer cycle in $G$, or

- Vertex cover of $G-B$ of size at most $\delta(G-B)+2 k$, or 
- Two paths $P_{1}, P_{2}$ that induce a Dirac decomposition for $C$ and $B$ in $G$.

Before proceeding with the proof of the lemma, we show how to use it for the proof of Theorem 3 .

\subsection{Proof of Theorem 3}

We combine the main results of Sections 7, 8, 9, and Lemma 20. Let $(G, B, k)$ be an instance of LONG Dirac CyCLE. First we consider the cases that do not fit the conditions of Lemma 20, If $\delta(G-B)<12$ or if $24 k>\delta(G-B)$, we can find a cycle of length at least $2 \delta(G-B)+k>48 k+24$ in time $2^{\mathcal{O}(k)} \cdot n^{\mathcal{O}(1)}$ by calling the algorithm for LONGEST CYCLE from Theorem 12 .

If $2 k+2|B|+12>\delta(G-B)$, we have that $5 k+4|B|+48>2 \delta(G-B)+k$. By Theorem 12, a cycle of length at least $5 k+4|B|+48$ could be found in time $2^{\mathcal{O}(k+|B|)} \cdot n^{\mathcal{O}(1)}$. If $\delta(G-B) \geq \frac{n}{2}-\frac{|B|+k}{2}$, we apply the algorithmic results of Section 8. We put $k^{\prime}=\max \left\{|B|, \frac{|B|+k}{2}\right\}$, and obtain that $\delta(G-B) \geq \frac{n}{2}-k^{\prime}$ for $|B| \leq k^{\prime}$. We apply Theorem 7 for $G, B$ and $k^{\prime}$. Then the problem is solvable in time $2^{\mathcal{O}\left(k^{\prime}\right)} \cdot n^{\mathcal{O}(1)}=2^{\mathcal{O}(k+|B|)} \cdot n^{\mathcal{O}(1)}$.

From now we assume that $k$ and $B$ satisfy the conditions of Lemma 20. In particular, now $\min \{2 \delta(G-B), n-|B|\}=2 \delta(G-B)$, so we are looking for a cycle of length at least $2 \delta(G-B)+k$ for $k \geq 0$. By Lemma 20 in polynomial time we either find a longer cycle, a vertex cover, or a Dirac decomposition of $G$. If a longer cycle is found and the length of this cycle is still less than $2 \delta(G-B)+k$, we call Lemma 20 with the longer cycle. If a vertex cover of $G-B$ of size at most $\delta(G-B)+2 k$ is found, then the vertex cover of $G$ is at most $\delta(G-B)+2 k+|B|$. We apply Theorem 6 to solve the problem in time $2^{\mathcal{O}(k+|B|)} \cdot n^{\mathcal{O}(1)}$. Finally, if a Dirac decomposition for $C$ and $B$ is found in $G$, we use Theorem 16 to solve $(G, B, k)$ in running time single-exponential in $k+|B|$ or find a longer cycle in $G$ and repeat the application of Lemma 20 .

The proof of Theorem 3 (up to the proof of Lemma 20) is complete.

\subsection{Last piece: proof of Lemma 20}

The remaining part of the section is devoted to the postponed proof of Lemma 20.

Proof of Lemma 20. The proof is algorithmic. We try to replace an $\operatorname{arc}$ of $C$, that is, a path in $C$, with a path in $G-V(C)$. This process of enlarging $C$ is similar to the process of enlarging a path in Lemma 4. We consider connected components $H$ in $G-V(C)$ that contain at least one vertex in $V(G) \backslash B$. Note that at least one such component exists since by the conditions of the lemma, $V(G-V(C)-B) \geq n-(2 \delta(G-B)+k-1)-|B|>1$.

To simplify our job, we first apply $B$-refinements to all connected components in $G-B$. Without loss of generality, we assume that $G$ is a graph with all possible $B$-refinements applied, i.e., $R_{B}(H)=$ $G$ for any connected component $H$ in $G-V(C)$ with $V(H) \nsubseteq B$. Note that this assumption preserves all resulting points of the lemma statement: if a longer cycle, or a vertex cover, or a Dirac decomposition is found for the graph with applied $B$-refinements, they can be easily restored in the original graph.

Similarly to the proof of Lemma 4, we consider several cases depending on the structure of a connected component $H$ with $V(H) \nsubseteq B$. The difference is that isolated vertices in $G-V(C)$ now do not lead to an immediate enlargement of $C$. However, we show that they contribute to a construction of a vertex cover of $G-B$.

In what follows we prove the following. If there is a component $H$ with $G-V(C)$ with exactly two vertices, then cycle $C$ can be always enlarged. If there is a component $H$ with at least 3 vertices, call it a large component, then either $C$ can be enlarged, or $H$ has a very special structure. The special structure of large components is used twice. First, we show that if there is at least one 
single-vertex component and at least one large component, then $C$ can be enlarged. Thus if we cannot enlarge $C$, it means that either $G-V(C)$ is an independent set or all components are large. In the first case, we prove that the vertex cover of $G-B$ is at most $\delta(G-B)+2 k$. In the second case, the structural properties of large components are used to construct a Dirac decomposition.

We start with two claims that will be used in several places of the proof. The first claim shows that if there is a pair of distant consecutive neighbors of a vertex $h \notin V(C)$ in $C$, then $C$ can be enlarged.

Claim 16. Let $h \in V(G) \backslash V(C)$ be a vertex with at least $\delta(G-B)-2$ neighbors in $V(C)$ and such that there is a pair of neighbors $u, v$ of $h$ on $C$ such that one of the $(u, v)$-arcs is of length at least $8 k$ containing no other neighbors of $h$. Then $C$ can be enlarged in polynomial time.

Proof of Claim 16. Suppose that there are two neighbors of $h$, say $u, v \in V(C)$ such that one arc of $C$ between $u$ and $v$ is of length at least $8 k$ and does not contain any neighbor of $h$. Hence the other arc between $u$ and $v$ contains all neighbors of $h$ on $C$. Moreover, since the length of $C$ is at most $2 \delta(G-B)+k-1$, the length of this arc is at most $2 \delta(G-B)-7 k-1$.

There are at least $\delta(G-B)-2$ neighbors of $h$ on $C$. Since $2(\delta(G-B)-3)>2 \delta(G-B)-7 k-1$, by the pigeonhole principle, there is a pair of neighbors of $h$ that are adjacent $C$. Then $h$ can be inserted in $C$ between these neighbors so the length of $C$ increases by one.

The following claim allows to eliminate the existence of large connected components in $G-V(C)$, when there are isolated vertices in $G-V(C)$. This claim will be useful later in this proof. Recall that a chord of a cycle $C$ is a path connecting two vertices of $C$ and containing no other vertices of $C$.

Claim 17. If there is a vertex $h \in V(G) \backslash V(C)$ with at least $\delta(G-B)$ neighbors in $V(C)$ and there is a chord of $C$ of length at least $16 k$ that does not pass through $h$, then $C$ can be enlarged in polynomial time.

Proof of Claim 17. By Claim 16, we can assume that for every pair of neighbors $u, v$ of $h$ on $C$, each of the $(u, v)$-arcs is either of length less than $8 k$ or contains other neighbors of $h$.

Let the endpoints of the chord be $c_{1}, c_{2} \in V(C)$. If the distance between $c_{1}$ and $c_{2}$ in $C$ is less than the length of the chord, then $C$ can be made longer by replacing an arc between $c_{1}$ and $c_{2}$ with the chord. Otherwise, both arcs between $c_{1}$ and $c_{2}$ are of length at least $16 k$.

Each of these two arcs should contain a neighbor of $h$ as an internal vertex. Select one of the two arcs between $c_{1}$ and $c_{2}$. Let $v_{1} \neq c_{1}$ be the neighbor of $h$ that is closest to $c_{1}$ on this arc. Since there are no other neighbors of $h$ between $c_{1}$ and $v_{1}$, the distance in $C$ between $c_{1}$ and $v_{1}$ is at most $8 k$. Analogously, take the other arc between $c_{1}$ and $c_{2}$ and let $v_{2}$ be the neighbor of $h$ on this arc that is closest to $c_{2}$, but is different from it. Again, the distance between $c_{2}$ and $v_{2}$ is at most $8 k$.

Now construct the following path between $v_{1}$ and $v_{2}$ : go from $v_{1}$ to $c_{2}$ following the first arc, then go from $c_{2}$ to $c_{1}$ following the chord, then go from $c_{1}$ to $v_{2}$ following the second arc. See Figure 11. This path contains all but at most $16 k$ edges of the cycle $C$, since $c_{i}$ and $v_{i}$ are close to each other on $C$ for each $i \in\{1,2\}$. Additionally, this path contains at least $16 k$ edges of the chord. Hence, the length of the constructed $\left(v_{1}, v_{2}\right)$-path is at least the length of the cycle $C$. This path does not contain $h$, so adding two edges between $v_{1}$ and $h$ and between $h$ and $v_{2}$ to it, yields a cycle of length at least $|V(C)|+2$.

Depending on the number of vertices in a component $H$ of $G-V(C)$, we consider difference cases. We start with the simplest case. 


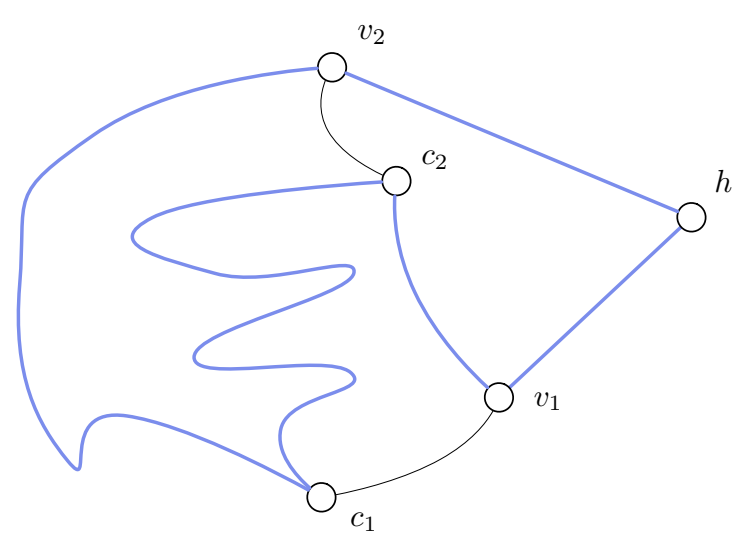

Figure 11: Rerouting through a chord.

Case 1: At least one component $H$ consists of two vertices. In this case we can always enlarge $C$ in polynomial time.

Let $V(H)=\left\{h_{1}, h_{2}\right\}$ for $h_{1} \neq h_{2}$. Then both $h_{1}$ and $h_{2}$ have at least $\delta(G-B)-1$ neighbors in $V(C)$ and are connected by an edge. In this case, $C$ can be made longer in polynomial time. We formulate this slightly more generally in the following claim.

Claim 18. If there are two distinct vertices $h_{1}, h_{2} \in V(G) \backslash V(C)$, each having at least $\delta(G-B)-1$ neighbors in $V(C)$, and that are connected by a path in $G-V(C)$, then the length of $C$ can be increased in polynomial time.

Proof of Claim 18. Let $S$ be the set of neighbors of $h_{1}$ and $h_{2}$ in $V(C)$. Let $a$ be the number of the common neighbors of $h_{1}$ and $h_{2}$ in $S$. Then $|S| \geq 2 \delta(G-B)-2-a$ and $S$ splits $C$ into at least $2 \max \{a, \delta(G-B)-1\}-a$ arcs. If we have an arc of length 1 , we can always enlarge $C$ by inserting one or both of the $h_{i}$. Moreover, if one of the endpoints of an arc is a common neighbor of $h_{1}$ and $h_{2}$, then the length of this arc should be at least 3. Indeed, if an arc having a common neighbor of $h_{1}$ and $h_{2}$ as its endpoint and is of length less than three, then we can insert a path between $h_{1}$ and $h_{2}$ and two boundary edges instead of this arc in $C$; thus $C$ becomes longer.

Therefore, if $C$ cannot be enlarged, its length is at least $2(|S|-a)+3 a$. By the conditions of the lemma, we have that $\delta(G-B) \geq 2 k+12$. If $a \geq \delta(G-B)-1$, then

$$
2(|S|-a)+3 a \geq 3 a \geq 3(\delta(G-B)-1)>2 \delta(G-B)+k .
$$

If $a<\delta(G-B)-1$, then

$$
2(|S|-a)+3 a=2|S|+a \geq 2(2 \delta(G-B)-2-a)+a=4 \delta(G-B)-a-8>2 \delta(G-B)+k .
$$

In both cases, we have that the length of cycle $C$ is more than $2 \delta(G-B)+k$. This contradicts our assumption that $|V(C)|<2 \delta(G-B)+k$.

The next two cases consider the situation when a component $H$ of $G-V(C)$ contains at least 3 vertices. Then $H$ could be 2 -connected or it contains a cut-vertex.

Case 2: $H$ is 2-connected. We show that either we can enlarge $H$, or $H$ has very specific properties described in Claim 19 and Claim 20. These properties will be used in handling isolated components and in constructing Dirac decomposition. 
Claim 19. Either the maximum size of a matching between $V(H)$ and $V(C)$ in $G$ is two, or $C$ can be enlarged in polynomial time.

Proof of Claim 19. Since $G$ is 2-connected, the maximum matching size between $V(H)$ and $V(C)$ is always at least 2.

Suppose first that at most one vertex in $V(H-B)$ has neighbors in $V(C)$. If such vertex exists, let $h \in V(H-B)$ be that vertex, otherwise let $h$ be an arbitrary vertex in $H-B$. We know that $\delta(H-(B \cup\{h\})) \geq \delta(G-B)-1$, since $H$ is a connected component in $G-V(C)$. We now claim that if there is a matching of size at least three between $V(H)$ and $V(C)$ in $G$, then $C$ can be made longer by replacing one of its arcs with a path in $H$. By Theorem 3 , there is a path of length at least $\delta(H-(B \cup\{h\})) \geq \delta(G-B)-1$ between an arbitrary pair of vertices in $H$. The endpoints of the matching in $V(C)$ split $C$ into at least three arcs. If at least one of these arcs is of length less than $(\delta(G-B)-1)+2$, it can be replaced with a path in $H$ connecting corresponding endpoints of the matching. Hence, if $C$ cannot be made longer, its length is at least $3 \delta(G-B)+3$. Since $|V(C)|<2 \delta(G-B)+k<3 \delta(G-B)+3$, we obtain that either $C$ can be made longer or the maximum matching size between $V(H)$ and $V(C)$ in $G$ is two.

Now we assume that at least two vertices in $V(H-B)$ have neighbors in $V(C)$. Take the vertices $h_{1}, h_{2} \in V(H-B)$ that have the most and the second most number of neighbors in $V(C)$. Denote $n_{i}=\left|N_{G}\left(h_{i}\right) \cap V(C)\right|$ for each $i \in\{1,2\}$. Thus $n_{1} \geq n_{2}$. By Theorem 3 , there is a path of length at least $\delta\left(H-\left(B \cup\left\{h_{1}\right\}\right)\right)$ between $h_{1}$ and $h_{2}$ in $H$. Let $t=\max \left\{\delta\left(H-\left(B \cup\left\{h_{1}\right\}\right)\right), 1\right\}$. Note that the path between $h_{1}$ and $h_{2}$ is of length at least $t$.

Assume that $\delta\left(H-\left(B \cup\left\{h_{1}\right\}\right)\right)<\delta(G-B)-1-n_{2}$. Then at least one vertex in $V\left(H-\left(B \cup\left\{h_{1}\right\}\right)\right)$ has at most $\delta(G-B)-2-n_{2}$ neighbors in $V\left(H-\left(B \cup\left\{h_{1}\right\}\right)\right)$. Hence, it has at most $\delta(G-B)-1-n_{2}$ neighbors in $V(H-B)$. All other neighbors of this vertex in $V(G-B)$ are from $V(C)$, so this vertex should have at least $n_{2}+1$ neighbors in $V(C)$. This contradicts the choice of $h_{2}$ and $n_{2}$. Thus, $t \geq \delta(G-B)-1-n_{2}$, or $n_{2} \geq \delta(G-B)-t-1$.

Denote by $S$ the set of all neighbors of $h_{1}$ and $h_{2}$ in $V(C)$, i.e. $S=\left(N_{G}\left(h_{1}\right) \cup N_{G}\left(h_{2}\right)\right) \cap V(C)$. Let $a$ be the number of common neighbors of $h_{1}$ and $h_{2}$ in $S$, i.e. $a=\left|N_{G}\left(h_{1}\right) \cap N_{G}\left(h_{2}\right) \cap S\right|$. Observe that vertices in $S$ split $C$ into $|S|=n_{1}+n_{2}-a$ arcs. Note that each arc is of length at least two, otherwise we enlarge $C$. Moreover, every arc whose endpoint is a common neighbor of $h_{1}$ and $h_{2}$ should have length at least $t+2$, because otherwise $C$ can be made longer. Hence, $|V(C)| \geq 2|S|+a t$. Since $2 \delta(G-B)+k>|V(C)|$, we have that

$$
2 \delta(G-B)+k>2\left(n_{1}+n_{2}-a\right)+a t \geq 4 n_{2}-2 a+a t \geq 4(\delta(G-B)-t-1)+a(t-2) .
$$

Therefore,

$$
k>2 \delta(G-B)-4 t-4+12-12+a(t-2)>2 \delta(G-B)-4(t-2)+a(t-2)-12,
$$

and hence

$$
(4-a)(t-2)>2 \delta(G-B)-k-12 .
$$

In particular, $(4-a)(t-2)>0$. If $t=1$, then $a>2 \delta(G-B)-k-8$. But $|V(C)| \geq 2|S|+a t \geq$ $2 a+a t=a(t+2) \geq 3 a$, so $3 a<2 \delta(G-B)+k$. It follows that $3(2 \delta(G-B)-k-8)<2 \delta(G-B)+k$, or $4 \delta(G-B)<4 k+24$, which contradicts the assumptions of the lemma.

Thus $t \neq 1$. Since $t-2 \neq 0$, we obtain that $t>2$, and, consequently, $a<4$. Then $3(t-2) \geq$ $(4-a)(t-2)>2 \delta(G-B)-k-12$, or $3 t>2 \delta(G-B)-k-6$. It yields that $t \geq \frac{1}{2} \delta(G-B)$.

Assume now that there is a matching in $G$ between $V(H)$ and $V(C)$ of size three. Let $c_{1}, c_{2}, c_{3}$ be the endpoints of this matching in $V(C)$, and $v_{1}, v_{2}, v_{3}$ be the corresponding endpoints in $V(H)$. 
Without loss of generality, we assume that $v_{1}=h_{2}$, as if $h_{2} \notin\left\{v_{1}, v_{2}, v_{3}\right\}$ we can always change the matching to include the vertex $h_{2}$. Denote by $T$ the set of all neighbors of $v_{1}, v_{2}$ and $v_{3}$ in $V(C)$, i.e., $T=N_{G}\left(\left\{v_{1}, v_{2}, v_{3}\right\}\right) \cap V(C)$. Note that $|T| \geq\left|N_{G}\left(v_{1}\right) \cap V(C)\right|=n_{2} \geq \delta(G-B)-t-1$. Unless $C$ can be made longer, the vertices of $T$ split $C$ into $|T|$ arcs of length at least two. Additionally, at least three arcs (the arcs that are incident to $c_{1}, c_{2}, c_{3} \in T$ ) should be of length at least $t+2$, as there is a path of length at least $t$ between $v_{i}$ and $v_{j}$ in $H$ for any $i \neq j$. We obtain that $|V(C)| \geq 2|T|+3 t \geq 2 \delta(G-B)+t-2>2 \delta(G-B)+k$ unless $C$ can be made longer.

Claim 20. Either between any pair of vertices in $H$ there is a path in $H$ of length at least $\delta(G-$ $B)-2$, or $C$ can be made longer in polynomial time.

Proof of Claim 20. The proof is identical to the proof of Claim 3.

Case 3: $|V(H)| \geq 3$ and $H$ contains a cut-vertex.

Since $H$ contains a cut-vertex, it contains at least two leaf-blocks. Denote the leaf-blocks of $H$ by $L_{1}, L_{2}, \ldots, L_{p}$ and their respective cut-vertices by $c_{1}, c_{2}, \ldots, c_{p}$, where $p \geq 2$.

Since $L_{i}$ is 2-connected or $\left|V\left(L_{i}\right)\right|=2$, we can proceed similarly to Case 2 with $L_{i}$ and $B \cup\left\{c_{i}\right\}$ instead of $H$ and $B$, and make $C$ longer or conclude that the maximum matching size between $V\left(L_{i}\right)$ and $V(C)$ in $G$ is at most two.

We now assume that for each $i \in[p]$ the maximum matching size between $V\left(L_{i}\right)$ and $V(C)$ is at most two. Then for any $i \in[p]$, accordingly to Claim 20 applied to $L_{i}$ and $B \cup\left\{c_{i}\right\}$ instead of $H$ and $B$, we obtain that there is a path of length at least $\delta\left(G-\left(B \cup\left\{c_{i}\right\}\right)-2 \geq \delta(G-B)-3\right.$ between any pair of vertices in $L_{i}$, if $\left|V\left(L_{i}\right)\right|>2$.

Claim 21. $\left|\bigcup_{i=1}^{p} N_{G}\left(V\left(L_{i}-\left\{c_{i}\right\}\right)\right)\right|=1$, or $C$ can be made longer in polynomial time.

Proof of Claim 21. We first show that if there exists $i \in[p]$ with $\left|V\left(L_{i}\right)\right|=2$, then $C$ can be made longer in polynomial time.

Assume that there exists $L_{i}$ with $\left|V\left(L_{i}\right)\right|=2$. Then $V\left(L_{i}\right)=\left\{u, c_{i}\right\}$ for some vertex $u \neq c_{i}$. As $R_{B}(H)=G$, it is true that $u \notin B$. Hence, $u$ has at least $\delta(G-B)-1$ neighbors in $V(C)$. If $u$ has two consecutive vertices of $C$ as neighbors, then $C$ can be made longer with inserting $u$.

Now take $j \in[p] \backslash\{i\}$ and consider the leaf-block $L_{j}$. If $\left|V\left(L_{j}\right)\right|=2$, then $V\left(L_{j}\right)=\left\{u^{\prime}, c_{j}\right\}$, where $u^{\prime}$ has at least $\delta(G-B)-1$ neighbors in $V(C)$. Note that $u$ and $u^{\prime}$ are connected by a path in $G-V(C)$. By Claim 18, $C$ can be made longer in polynomial time in this case.

If $\left|V\left(L_{j}\right)\right|>2$, then $L_{j}$ is 2-connected, so there is a path of length at least $\delta(G-B)-3$ between any pair of vertices in $L_{j}$. Hence, each inner vertex of $L_{j}$ is connected with $u$ by a path of length at least $\delta(G-B)-2$. Take a vertex $u^{\prime} \in V\left(L_{j}-\left\{c_{j}\right\}\right)$ that has a neighbor $v^{\prime} \in V(C)$. By Claim 16 . there is a vertex $v \in V(C)$ that is a neighbor of $u$ and is on a distance at least one and at most $8 k$ from $v^{\prime}$ on $C$. We obtain a $\left(v, v^{\prime}\right)$-chord of $C$ that is of length at least $\delta(G-B)$ but the distance between $v$ and $v^{\prime}$ on $C$ is at most $8 k<\delta(G-B)$. Hence, $C$ can be made longer in polynomial time.

We now assume that $\left|V\left(L_{i}\right)\right| \geq 3$ for each $i \in[p]$. Then there is a path of length at least $\delta(G-B)-3$ for any pair of vertices in any $L_{i}$. Assume that $\bigcup_{i=1}^{p} N_{G}\left(V\left(L_{i}-\left\{c_{i}\right\}\right)\right) \supseteq\left\{v_{1}, v_{2}\right\}$, where $v_{1}, v_{2} \in V(C)$ and $v_{1} \neq v_{2}$. Then either there exist $i \neq j$ such that $L_{i}-\left\{c_{i}\right\}$ contains a neighbor of $v_{1}$ and $L_{j}-\left\{c_{j}\right\}$ contain a neighbor of $v_{2}$, or there only exists $i$ such that $L_{i}-\left\{c_{i}\right\}$ contains both a neighbor of $v_{1}$ and a neighbor of $v_{2}$. In the latter case, we can pick $j \neq i$ and $v_{3} \in V(C)$ with $v_{3} \neq v_{1}$ or $v_{3} \neq v_{2}$ such that $L_{j}-\left\{c_{j}\right\}$ contains a neighbor of $v_{3}$. Thus, without loss of generality we assume that $L_{1}-\left\{c_{1}\right\}$ contains a neighbor $u_{1}$ of $v_{1} \in V(C)$ and $L_{2}-\left\{c_{2}\right\}$ contains a neighbor $u_{2}$ of $v_{2} \in V(C)$ and $v_{1} \neq v_{2}$. 
Observe that there exists a $\left(u_{1}, u_{2}\right)$-path in $H$ of length at least $2 \delta(G-B)-6$. Hence, this path can be prolonged to a $\left(v_{1}, v_{2}\right)$-chord of $C$ of length at least $2 \delta(G-B)-4$. Note that at least one of $\left(v_{1}, v_{2}\right)$-arcs of $C$ is of length at most $\delta(G-B)-\frac{k-1}{2}<2 \delta(G-B)-4$, so $C$ can be made longer in polynomial time.

The following claim shows that $H$ yields at least one long chord of $C$.

Claim 22. Either for any $i \in[p]$ and any $u \in V\left(L_{i}-c_{i}\right), v \in V(H) \backslash u$, there is a $(u, v)$-path of length at least $\delta(G-B)-2$ in $H$, or $C$ can be made longer in polynomial time.

Proof of Claim 22. Take $i \in[p]$. From Claim 21 follows that $\delta\left(L_{i}-\left(B \cup\left\{c_{i}\right\}\right)\right) \geq \delta(G-B)-2$, as each vertex in $V\left(L_{i}-c_{i}\right)$ has at most one neighbour outside $L_{i}$. By Corollary 3 , there is a path of length at least $\delta(G-B)-2$ between any pair of vertices inside $L_{i}$.

Take $u \in V\left(L_{i}-c_{i}\right)$ and $v \in V(H) \backslash u$. If $v \in V\left(L_{i}\right)$, then we are done. If $v$ is outside $L_{i}$, then a path between $u$ and $v$ should go through $c_{i}$. Since $u \neq c_{i}$, there is a $\left(u, c_{i}\right)$-path of length at least $\delta(G-B)-2$ inside $L_{i}$. Combine this path with any $\left(c_{i}, v\right)$-path outside $L_{i}$ in $H$ to obtain the required $(u, v)$-path.

Case 4: At least one component $H$ of $G-V(C)$ consists of one vertex. In this case we show that either we can enlarge $C$ in polynomial time, or construct a vertex cover of $G-B$ of size at most $\delta(G-B)+2 k$.

Let $V(H)=\{h\}$ for some vertex $h \in V(G-B)$. All neighbors of $h$ are from $V(C)$, so $h$ has at least $\delta(G-B)$ neighbors in $V(C)$.

We first claim that if $G-V(C)$ contains both an isolated vertex and some non-isolated connected component, then we can make $C$ longer.

Claim 23. Let $H_{1}$ and $H_{2}$ be two connected components in $G-V(C)$ with $V\left(H_{i}\right) \nsubseteq B$. If $\left|V\left(H_{1}\right)\right|=$ 1 and $\left|V\left(H_{2}\right)\right| \neq 1$, then $C$ can be made longer in polynomial time.

Proof of Claim 23. We can assume that $\left|V\left(H_{2}\right)\right| \geq 3$, so $V\left(H_{2}\right)$ is either 2-connected or contains a cut-vertex. In both of the cases, by Claim 20 and Claim 22, we can find a chord of $C$ of length at least $\delta(G-B)-2>16 k$ that passes through $H_{2}$. By Claim 17, the single vertex of $H_{1}$ and the chord passing through $H_{2}$ help making $C$ longer in polynomial time.

By Claim 23, we can assume that if there is one connected component of $G-V(C)$ which is an isolated vertex, then all other components are also isolated vertices.

Our next step is to to show that if an isolated vertex exists, then we can find a large independent set in $C$ that has no neighbors outside $C$. For an isolated vertex $h$ in $G-V(C)$, we define the set of its 101-neighbors. A vertex $v \in V(C)$ is a 101-neighbor of $h$, if it is not a neighbor of $h$, i.e., $v \notin N_{G}(h)$, but both neighbors of $v$ in $C$ are also the neighbors of $h$. In other words, the set of all 101-neighbors of $h$ is the set of all isolated vertices in $C-N_{G}(h)$. We now claim that if $C$ cannot be enlarged, then 101-neighbors of a vertex $h$ form an independent set in $C$ and do not have neighbors in $V(G) \backslash V(C)$.

Claim 24. Let $h \notin B$ be an isolated vertex in $G-V(C)$. If at least one 101-neighbor of $h$ on $C$ is not in $B$ and has at least one neighbor in $V(G-V(C)-B)$ or two 101-neighbors of h are connected by an edge, then $C$ can be made longer in polynomial time.

Proof of Claim 24. Suppose first that two 101-neighbors of $h$, say $v_{1}, v_{2} \in V(C)$, are connected by an edge in $G$. Let the neighbors of $v_{i}$ on $C$ be $u_{i}$ and $w_{i}$ for $i \in\{1,2\}$. Without loss of generality, we assume that the six vertices appear in the order $u_{1}, v_{1}, w_{1}, u_{2}, v_{2}, w_{2}$ when following $C$, and 


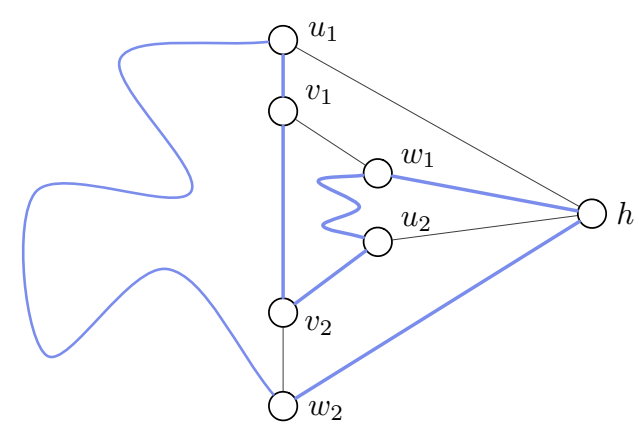

Figure 12: Rerouting through adjacent 101-neighbors.

possibly $w_{1}=u_{2}$ or $w_{2}=u_{1}$. Then construct a new cycle as following: $u_{1} \rightarrow v_{1} \rightarrow v_{2} \rightarrow u_{2} \rightsquigarrow$ $w_{1} \rightarrow h \rightarrow w_{2} \rightsquigarrow u_{1}$, where $\rightarrow$ corresponds to following a single edge in $G$, while $\rightsquigarrow$ corresponds to following an arc of $C$. See Figure 12. Note that the vertex set of the new cycle is $V(C) \cup\{h\}$, so $C$ is enlarged in this case.

Now suppose that a 101-neighbor of $h$, say $v \in V(C)$, has a neighbor outside $V(C)$, say $h^{\prime} \in$ $V(G-V(C)-B)$. By Claim 23, we can assume that all vertices in $G-V(C)$ are isolated. Assume that $h^{\prime}$ is the only neighbor of $v$ in $V(G-V(C))$. Then replace $v$ with $h$ in $C$, so $v$ becomes a vertex outside $C$. Then $v$ and $h^{\prime}$ form a connected component of size two in $G-V(C)$. Since $v \notin B, v$ has at least $\delta(G-B)-1$ neighbors in $V(C)$. By Claim 18, $C$ can be made longer in polynomial time.

If $h^{\prime}$ is not the only neighbor of $v$, then after the replacement $v$ connects two vertices with at least $\delta(G-B)-1$ neighbors in $V(C)$. We can again apply Claim 18 and make $C$ longer.

Constructing vertex cover. The construction of vertex cover of $G-B$ of size at most $\delta(G-B)+$ $2 k$ is possible when there is at least one isolated vertex in $G-V(C)$. Take an isolated vertex $h$ in $G-V(C)-B$. Denote by $a$ the number of its 101-neighbors. The neighbors of $h$ on $C$ split $C$ into arcs. Since each 101-neighbor corresponds to an arc of length two, and all other arcs are of length at least three, we obtain that $2 a+3(\delta(G-B)-a) \geq|V(C)|$, so $a \geq 3 \delta(G-B)-|V(C)|>\delta(G-B)-k$. Now denote by $S$ the set of all 101-neighbors of an isolated vertex in $G-V(C)$, so $|S|=a$. By Claim 24, $(V(G) \backslash V(C)) \cup(S \backslash B)$ is an independent set in $G$, so $V(C) \backslash(S \cup B)$ is a vertex cover of $G-B$. The size of this vertex cover is at most $(2 \delta(G-B)+k-1)-(\delta(G-B)-k+1)<\delta(G-B)+2 k$. Finally, the the desired vertex cover of $G-B$ can be trivially found in polynomial time by taking an isolated component $h$ and constructing the set of its 101-neighbors.

Constructing Dirac decomposition. When no isolated vertex is presented in $G-V(C)-B$, then $G-V(C)$ consists of non-empty connected components, apart from components that are completely contained in $B$. We show how to construct a Dirac decomposition in this case. Before proceeding with claims, it is convenient to define the following notion agreeing with the definition of Dirac decompositions.

Definition 7 (Dirac layouts). We say that a vertex set $X \subseteq V(C)$ is in Dirac layout on $C$, if the vertices of $X$ split $C$ into arcs such that two of these arcs are of length at least $\delta(G-B)$.

In what follows, we show that neighbors of $V(G-V(C))$ on $C$ are in Dirac layout, unless $C$ can be made longer. We start showing this first for every connected component in $G-V(C)$.

Claim 25. Let $H$ be a connected component in $G-V(C)$ with $|V(H)| \geq 3$ and $V(H) \nsubseteq B$. If $N_{G}(V(H))$ is not in Dirac layout on $C$, then $C$ can be made longer in polynomial time. 
Proof of Claim 25. Denote $S=N_{G}(V(H))$. Note that $S \subseteq V(C)$. We know that $S$ splits $C$ into $|S|$ arcs. Denote $S=\left\{v_{1}, v_{2}, \ldots, v_{t}\right\}$, where $v_{1}, v_{2}, \ldots, v_{t}$ are the vertices of $S$ on $C$ in the order when following $C$ in some direction. We also assume that $v_{t+1}=v_{1}$.

Assume first that $H$ is 2-connected. Then assign to each vertex $v_{i} \in S$ a set of its neighbors in $H$. That is, make an assignment $\sigma: S \rightarrow 2^{V(H)}$ with $\sigma\left(v_{i}\right)=N_{G}\left(v_{i}\right) \cap V(H)$. As $G$ is 2-connected, $\left|\bigcup_{i=1}^{t} \sigma\left(v_{i}\right)\right| \geq 2$. If for at least one $i \in[t]$ holds $\left|\sigma\left(v_{i}\right)\right|=2$, then there exist at least two $j \in[t]$ with $\max \left\{\left|\sigma\left(v_{j}\right)\right|,\left|\sigma\left(v_{j+1}\right)\right|\right\} \geq 2$. For each such $j$, we can pick $h_{j} \in \sigma\left(v_{j}\right)$ and $h_{j+1} \in \sigma\left(v_{j+1}\right)$ with $h_{j} \neq h_{j+1}$. Since there is a path of length at least $\delta(G-B)-2$ in $H$, the length of the arc between $s_{j}$ and $s_{j+1}$ should be at least $\delta(G-B)$. Otherwise we can make $C$ longer.

Now consider that $\left|\sigma\left(v_{i}\right)\right|=1$ for each $i \in[t]$. But not all values of $\sigma\left(v_{i}\right)$ are equal, since their union is of size at least two. Then there exist at least two $j \in[t]$ with $\sigma\left(v_{j}\right) \neq \sigma\left(v_{j+1}\right)$. Hence, we can again assign distinct $h_{j}$ and $h_{j+1}$ and obtain that the $\left(v_{j}, v_{j+1}\right)$-arc should be of length at least $\delta(G-B)$.

It is left to consider the case when $H$ is not 2-connected. We again make an assignment $\sigma$, but now this assignment is slightly different and is denoted $\sigma: S \rightarrow 2^{\{0,1\}}$. If a vertex $v_{i}$ has a neighbor in $H$ that is an inner vertex of a leaf-block of $H$, then $1 \in \sigma\left(v_{i}\right)$. If $v_{i}$ has a neighbor in $H$ that is not an inner vertex of a leaf-block, put $0 \in \sigma\left(v_{i}\right)$. Thus, $\sigma\left(v_{i}\right)$ denotes the set of types of neighbors that $v_{i}$ has in $V(H)$. Note that $\bigcup_{i=1}^{t} \sigma\left(v_{i}\right)=\{0,1\}$ by Claim 21 and 2-connectivity of $G$. Analogously to the 2-connected case, there are two $j \in[t]$ with $0 \in \sigma\left(v_{j}\right)$ and $1 \in \sigma\left(v_{j+1}\right)$ or vice versa. Since there is a path of length at least $\delta(G-B)-2$ between any inner leaf-block vertex and any other vertex, we obtain that the arcs between $v_{j}$ and $v_{j+1}$ should be of length at least $\delta(G-B)$.

Claim 26. Assume that $G-V(C)$ contains no isolated vertex. Let $X$ be the union of vertex sets of all connected components $H$ in $G-V(C)$ with $V(H) \nsubseteq B$. If $N_{G}(X)$ is not in Dirac layout on $C$, then $C$ can be made longer in polynomial time.

Proof of Claim 26. Take a connected component $H$ in $G-V(C)$ with $V(H) \nsubseteq B$. By Claim 25, we assume that $N_{G}(V(H))$ is in Dirac layout on $C$. Hence, the vertices in $N_{G}(V(H))$ can be covered by two arcs of $C$ of total length at most $|V(C)|-2 \delta(G-B)$ and the distance between these arcs on $C$ is at least $\delta(G-B)$. Let $u_{1}, u_{2}$ and $v_{2}, v_{1}$ be the endpoints of these arcs. Among all possible ways to choose the arcs we choose the way when the total length of the $\left(u_{1}, u_{2}\right)$-arc and $\left(v_{2}, v_{1}\right)$-arc is the minimum possible. Hence, $u_{1}, u_{2}, v_{1}, v_{2} \in N_{G}(V(H))$ and the $\left(u_{1}, u_{2}\right)$-arc and the $\left(v_{2}, v_{1}\right)$-arc together contain all neighbors of $N_{G}(V(H))$ on $C$. Note that these arcs can be of zero length. For example, if $\left|N_{G}(V(H))\right|=2$, then $u_{1}=u_{2}$ and $v_{1}=v_{2}$, so $N_{G}(V(H))=\left\{u_{1}, v_{1}\right\}$.

We also assume that the order of the vertices on $C$ is $u_{1}, u_{2}, v_{2}, v_{1}$ when following $C$ in some direction. Thus, the chords between $u_{1}$ and $v_{1}$ and between $u_{2}$ and $v_{2}$ do not intersect graphically but can only coincide in one or two endpoints. From the proof of Claim 25 follows that $H$ yields a $\left(u_{1}, v_{1}\right)$-chord or a $\left(u_{2}, v_{2}\right)$-chord of $C$ of length at least $\delta(G-B)$.

Let $S$ be the union of the sets $\left\{u_{1}, u_{2}, v_{1}, v_{2}\right\}$ among all connected components of $G-V(C)$. It is easy to see that $S$ is in Dirac layout on $C$ if and only if $S$ is on Dirac layout on $C$. It is left to show that $S$ is in Dirac layout on $C$ or $C$ can be made longer in polynomial time.

Consider the vertices in $S$ on $C$. They are connected by chords of length at least $\delta(G-B)$ yielded by their connected components. If there is a pair of these chords that intersect graphically, then the chords in this pair correspond to distinct connected components of $G-V(C)$. Hence, if such a pair exists, we can enlarge $C$ as we did in the proof of Lemma 19. We can now assume that no two chords of $S$ intersect graphically. But we also know that no chord splits $C$ into two arcs such that one of them is shorter than $\delta(G-B)$. Hence, there are two arcs of length at least 


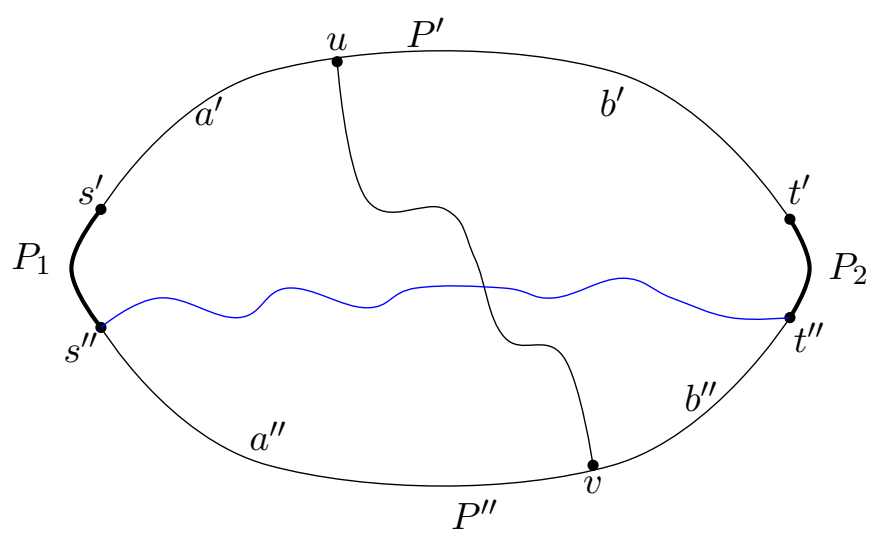

Figure 13: A schematic picture of an existence of a chord between $P^{\prime}$ and $P^{\prime \prime}$ passing through $B$. A blue chord represents a chord of $C$ passing through a component of $G-V(C)$.

$\delta(G-B)$ that do not contain any vertex in $S$ as inner vertex. Then $S$ is in Dirac layout on $C$ by definition.

The claim shows that $N_{G}(X)$ can be covered by two arcs of $C$ of total length at most $k-1$ at a distance at least $\delta(G-B)$ between them. Let $P_{1}$ and $P_{2}$ be these two arcs chosen in the unique way that minimizes their total length. It is left for us to show that $P_{1}$ and $P_{2}$ induce a Dirac component for $C$ and $B$ in $G$.

The first property from the defition of Dirac component is satisfied by the way $P_{1}$ and $P_{2}$ are constructed. It is easy to verify the second property for each connected component in $G-V(C)$ : 2-connected components form (D1)-type components and components containing cut vertices form (D2) and (D3)-type components of the Dirac decomposition. If the matching size conditions are not satisfied for one of these components, then $C$ can be trivially made longer in polynomial time using a long chord yielded by the component.

It is important to verify that the second property holds for all connected components in $G-$ $V\left(P_{1} \cup P_{2}\right)$. Note that a connected component $H$ in $G-V(C)$ with $V(H) \nsubseteq B$ is a connected component in $G-V\left(P_{1} \cup P_{2}\right)$ as well. Connected components that appear in $G-V(C)$ but do not appear in $G-V\left(P_{1} \cup P_{2}\right)$ are connected components that contain vertices in $V(C) \backslash V\left(P_{1} \cup P_{2}\right)$.

Note that there is either one or two such connected components, because the vertex set $V(C) \backslash$ $V\left(P_{1} \cup P_{2}\right)$ is a union of vertex sets of two arcs of $C$. If there is just one such connected component $H$, then $V(C) \backslash V\left(P_{1} \cup P_{2}\right) \subseteq V(H)$. We claim that if such $H$ exists in $G-V\left(P_{1} \cup P_{2}\right)$, then $C$ can be made longer in polynomial time (except in some very specific cases).

Assume that such $H$ exists. Then two arcs of $C$ of length at least $\delta(G-B)$ (denoted by $P^{\prime}$ and $P^{\prime \prime}$ in the definition of Dirac decomposition and here) are connected by a chord that can pass internally only through vertices in $B$. Note that the length $\delta(G-B)$ does not match the lower bound in the definition of Dirac decompositions. This is intentional. In one of the cases below, we have to expand the paths $P_{1}$ and $P_{2}$ and reduce the length of $P^{\prime}$ and $P^{\prime \prime}$ by one or two.

Denote by $s^{\prime}$ and $t^{\prime}$ and by $s^{\prime \prime}$ and $t^{\prime \prime}$ the endpoints of the $\operatorname{arcs} P^{\prime}$ and $P^{\prime \prime}$ respectively. Note that $V\left(P^{\prime}-\left\{s^{\prime}, t^{\prime}\right\}\right) \cup V\left(P^{\prime \prime}-\left\{s^{\prime \prime}, t^{\prime \prime}\right\}\right) \subseteq V(H)$, but $s^{\prime}, t^{\prime}, s^{\prime \prime}, t^{\prime \prime} \notin V(H)$. Since $P_{1}$ and $P_{2}$ are an $\left(s^{\prime}, s^{\prime \prime}\right)$-arc and an $\left(t^{\prime}, t^{\prime \prime}\right)$-arc of $C$ respectively. Hence, the chord connecting $P^{\prime}$ and $P^{\prime \prime}$ has endpoints in inner vertices of $P^{\prime}$ and $P^{\prime \prime}$. For clarity of presentation, we formulate the following intermediate claim. 
Claim 27. If there exists a connected component $H$ in $G-V\left(P_{1} \cup P_{2}\right)$ with $V(C) \backslash V\left(P_{1} \cup P_{2}\right) \subseteq$ $V(H)$, then either the only chords connecting $P^{\prime}-\left\{s^{\prime}, t^{\prime}\right\}$ and $P^{\prime \prime}-\left\{s^{\prime \prime}, t^{\prime \prime}\right\}$ are between their respective endpoints or $C$ can be made longer in polynomial time.

Proof of Claim 27. Let $u \in V\left(P^{\prime}-\left\{s^{\prime}, t^{\prime}\right\}\right)$ and $v \in V\left(P^{\prime \prime}-\left\{s^{\prime \prime}, t^{\prime \prime}\right\}\right)$ be the endpoints of this chord. Denote by $a^{\prime}$ and $b^{\prime}$ the length of the paths that $u$ splits $P^{\prime}$ into. Analogously, by $a^{\prime \prime}$ and $b^{\prime \prime}$ denote the length of the paths that $v$ splits $P^{\prime \prime}$ into, as shown in Figure 13 . If $\max \left\{a^{\prime}+b^{\prime \prime}, a^{\prime \prime}+b^{\prime}\right\}+\delta(G-B) \geq$ $|V(C)|$, then we can find a cycle longer than $C$ in polynomial time using a chord passing though some connected component in $G-V(C)$ and the $(u, v)$-chord of $C$.

Note that if $u$ is the neighbor of $s^{\prime}$ in $P^{\prime}$ and $v$ is the neighbor of $t^{\prime \prime}$ in $P_{2}$, then $b^{\prime} \geq \delta(G-B)-1$ and $a^{\prime \prime} \geq \delta(G-B)-1$ so $a^{\prime \prime}+b^{\prime}+\delta(G-B)>|V(C)|$ and $C$ can be made longer. The situation when $u$ is the neighbor of $t^{\prime}$ and $v$ is the neighbor of $s^{\prime \prime}$ is symmetrical.

We now assume that $a^{\prime}+b^{\prime \prime}<\delta(G-B)+k$ and $a^{\prime \prime}+b^{\prime}<\delta(G-B)+k$ (and, consequently, $a^{\prime}+b^{\prime \prime} \geq \delta(G-B)$ and $\left.a^{\prime \prime}+b^{\prime} \geq \delta(G-B)\right)$ for each choice of $u$ and $v$. That is, each such $(u, v)$-chord should split $C$ in a way that the difference between $a^{\prime}+b^{\prime \prime}$ and $a^{\prime \prime}+b^{\prime}$ is at most $k$.

Consider a fixed $u \in V\left(P^{\prime}\right)$. Without loss of generality, we assume that $u$ is not the neighbor of $s^{\prime}$ in $P^{\prime}$. Note that distinct choices of $v \in V\left(P^{\prime \prime}\right)$ provides distinct values of $a^{\prime \prime}$ and $b^{\prime \prime}$ with fixed sum. Hence, if there are at least $2 k+1$ choices of a pair $(u, v)$ for a fixed $u$, there are $2 k+1$ different values of $a^{\prime}+b^{\prime \prime}$. Since the sum of $a^{\prime}, b^{\prime}, a^{\prime \prime}, b^{\prime \prime}$ is also fixed, in at least one of these choices the difference between $a^{\prime}+b^{\prime \prime}$ and $a^{\prime \prime}+b^{\prime}$ is at least $k+1$. It follows that if $u$ has at least $2 k+1$ neighbors in $V\left(P^{\prime \prime}\right)$, then $C$ can be made longer in polynomial time. Note that the same arguments apply to a fixed choice of $v \in V\left(P^{\prime \prime}\right)$.

We have that for each $u \in V\left(P^{\prime}\right),\left|N_{G}(u) \cap V\left(P^{\prime \prime}\right)\right| \leq 2 k$. As soon as vertices in $P^{\prime}-\left\{s^{\prime}, t^{\prime}\right\}$ can have neighbors outside only in $V\left(P^{\prime \prime}\right), V\left(P_{1} \cup P_{2}\right)$ and $B$, we have that, $\delta\left(G\left[V\left(P^{\prime}-\left\{s^{\prime}, t^{\prime}\right\}\right)\right]-\right.$ $B) \geq \delta(G-B)-\left|V\left(P_{1} \cup P_{2}\right)\right|-2 k$. Since the total length of $P_{1}$ and $P_{2}$ is at most $k-1$ and $\left|V\left(P_{1} \cup P_{2}\right)\right| \leq k+2$, we have that $\delta\left(G\left[V\left(P^{\prime}-\left\{s^{\prime}, t^{\prime}\right\}\right)\right]-B\right) \geq \delta(G-B)-3 k-1$. Denote by $H^{\prime}$ the graph $G\left[V\left(P^{\prime}-\left\{s^{\prime}, t^{\prime}\right\}\right)\right]-B$. As the length of $P^{\prime}$ is less than $\delta(G-B)+k$, we have that $\left|V\left(H^{\prime}\right)\right| \leq \delta(G-B)+k-2$. Hence, $\delta\left(H^{\prime}\right)>\left|V\left(H^{\prime}\right)\right|-4 k$. On the other hand, $\delta\left(H^{\prime}\right) \geq \delta(G-B)-4 k \geq 20 k$.

We can now apply Lemma 15 to $H^{\prime}$ with $p=4 k, r=1$ and $\left\{s_{1}, t_{1}\right\}=\left\{u^{\prime}, u\right\}$, where $u^{\prime}$ is the neighbor of $s^{\prime}$ in $P^{\prime}$. Note that $u^{\prime} \neq u$ by our assumption. By Lemma 15 , there is a Hamiltonian $\left(u^{\prime}, u\right)$-path in $H^{\prime}$. This path is of length at least $\delta(G-B)-2-|B|$ that is found in polynomial time. Hence, we obtain a $\left(s^{\prime}, u\right)$-path of length at least $\delta(G-B)-1-|B|$ that contains only vertices of $P^{\prime}-t^{\prime}$.

The arguments of constructing a $\left(s^{\prime}, u\right)$-path for $P^{\prime}$ are applicable for constructing a $\left(t^{\prime \prime}, v\right)$-path for $P^{\prime \prime}$, if $v$ is not the neighbor of $t^{\prime \prime}$ in $P^{\prime \prime}$. Then we are able to construct a $\left(t^{\prime \prime}, v\right)$-path of length at least $\delta(G-B)-1-|B|$. Combine the $\left(s^{\prime}, u\right)$-path with $P_{1}$ and the $\left(t^{\prime \prime}, v\right)$-path and two chords: the $(u, v)$-chord and a $\left(s^{\prime \prime}, t^{\prime \prime}\right)$-chord of length at least $\delta(G-B)$ (it is depicted in Figure 13) to obtain a cycle of length at least $3 \delta(G-B)-2|B|-1 \geq 2 \delta(G-B)+k$. The last chord always exists by the construction of $P_{1}$ and $P_{2}$.

Note that we only required in the above construction that if $v$ is not the neighbor of $t^{\prime \prime}$ in $P^{\prime \prime}$. If $v$ is the neighbor of $t^{\prime \prime}$, then we can consider constructing a $\left(t^{\prime \prime}, u\right)$-path instead of $\left(s^{\prime}, u\right)$-path, but only if $u$ is not the neighbor of $t^{\prime}$ in $P^{\prime}$. The long path between $s^{\prime \prime}$ and $v$ required for construction is then given by $P^{\prime \prime}$, and is of length $a^{\prime \prime} \geq \delta(G-B)-1$.

We are left with the cases when $u$ and $v$ are simultaneously the neighbors of $t^{\prime}$ and $t^{\prime \prime}$ in $P^{\prime}$ and $P^{\prime \prime}$ respectively, or the neighbors of $s^{\prime}$ and $s^{\prime \prime}$ in $P^{\prime}$ and $P^{\prime \prime}$ respectively. That is, the cases when $b^{\prime}=b^{\prime \prime}=1$ or $a^{\prime}=a^{\prime \prime}=1$. In these cases, we cannot construct a pair of long paths and combine 
them with two chords, because we cannot apply Lemma 15 to both $u$ (e.g. to $\left\{u^{\prime}, u\right\}$, as $u^{\prime}=u$ ) and $v$. In other cases, we can make $C$ longer in polynomial time.

We now assume that the only two $(u, v)$-chords between $P^{\prime}$ and $P^{\prime \prime}$ can be only a chord between the neighbor of $s^{\prime}$ in $P^{\prime}$ and the neighbor of $s^{\prime \prime}$ in $P^{\prime \prime}$ and a chord between the neighbor of $t^{\prime}$ in $P^{\prime}$ and the neighbor of $t^{\prime \prime}$ in $P^{\prime \prime}$. In this case, we need to expand $P_{1}$ or $P_{2}$ to contain two more vertices. If there is a chord between the neighbors of $s^{\prime}$ and $s^{\prime \prime}$, expand $P_{1}$ with two edges so it starts containing these neighbors. Analogously, expand $P_{2}$ if there is a chord between the neighbors of $t^{\prime}$ and $t^{\prime \prime}$.

Observe that such expansion of $P_{1}$ or $P_{2}$ with two edges does not influence the properties for connected components in $G-V(C)$. We now have that $V\left(P^{\prime}-\left(V\left(P_{1}\right) \cup V\left(P_{2}\right)\right)\right)$ and $V\left(P^{\prime \prime}-\right.$ $\left.\left(V\left(P_{1}\right) \cup V\left(P_{2}\right)\right)\right)$ belong to distinct connected components in $G-\left(V\left(P_{1}\right) \cup V\left(P_{2}\right)\right)$. The length of $P^{\prime}$ and $P^{\prime \prime}$ is now at least $\delta(G-B)-2$ and the total length of $P_{1}$ and $P_{2}$ is at most $k+4$. The first and the last properties of a Dirac decomposition are satisfied by $P_{1}$ and $P_{2}$.

Denote the two connected components of $G-\left(V\left(P_{1}\right) \cup V\left(P_{2}\right)\right)$ that contain inner vertices of $P^{\prime}$ and $P^{\prime \prime}$ by $H^{\prime}$ and $H^{\prime \prime}$ respectively. We know that $\left|V\left(H^{\prime}\right)\right|,\left|V\left(H^{\prime \prime}\right)\right| \leq \delta(G-B)+|B|$ while $\delta\left(H^{\prime}-B\right) \geq \delta(G-B)-\left|V\left(P_{1}\right) \cup V\left(P_{2}\right)\right| \geq \delta(G-B)-k-6 \geq \frac{1}{2} \delta(G-B)+|B|$. Consider the $B$-refinements of $H^{\prime}$ and $H^{\prime \prime}$. If one of them is not 2-connected, then it should contain two leaf-blocks each consisting of at least $\frac{1}{2} \delta(G-B)+|B|+1$ vertices. Then, the total number of vertices in this component would be $2\left(\frac{1}{2} \delta(G-B)+|B|+1\right)-1>\delta(G-B)+|B|$, which is not possible. Hence, the $B$-refinements of $H^{\prime}$ and $H^{\prime \prime}$ are 2-connected. It is left to prove that they satisfy the properties of (D1)-type components of Dirac decompositions.

That is, we have to prove that the maximum matching size between $V\left(H^{\prime}\right)$ or $V\left(H^{\prime \prime}\right)$ and $V\left(P_{1}\right)$ or $V\left(P_{2}\right)$ is exactly one after the $B$-refinements. Consider that the matching size between $V\left(H^{\prime}\right)$ and $V\left(P_{1}\right)$ equals two. If the path $P_{1}$ was not expanded, then there is a long chord of $C$ passing though a component in $G-V(C)$ and connecting $s^{\prime}$ with a vertex in $P_{2}$. Hence, we can take a cycle of length at least $2 \delta(G-B)-2$ combined of this chord, $P^{\prime \prime}, P_{1}$ and a part of $P_{2}$. Then Corollary 3 and the maximum matching between $V\left(H^{\prime}\right)$ and $V\left(P_{1}\right)$ yields a chord of this cycle with endpoints in $V\left(P_{1}\right)$ of length at least $\delta\left(H^{\prime}-B\right)+2 \geq \delta(G-B)-k-4$. Since the length of $P_{1}$ is at most $k$, we can enlarge this cycle and obtain a cycle of length at least $(2 \delta(G-B)-2)+(\delta(G-B)-k-4)-k \geq 3 \delta(G-B)-2 k-6>2 \delta(G-B)+k$.

If $P_{1}$ was expanded, then there is no long chord of $C$ connecting the common endpoint of $P_{1}$ and $P^{\prime}$ with a vertex in $P_{2}$. However, then there exists a short chord of $C$ connecting the endpoints of $P_{1}$ and passing only through $B$ without visiting $H^{\prime}$ or $H^{\prime \prime}$ or any component in $G-V(C)$. Also, there is still a chord connecting $s^{\prime}$ with some vertex in $V\left(P_{2}\right)$, though $s^{\prime}$ now is not an endpoint of $P_{1}$ but the neighbor of the common endpoint of $P_{1}$ and $P^{\prime}$ in $P_{1}$. If the endpoints of the matching in $V\left(P_{1}\right)$ do not include either $s^{\prime}$ or the endpoint of $P_{1}$, we can proceed in the same way as when $P_{1}$ was not expanded. As $V\left(H^{\prime}\right)$, the matching and the edge between $s^{\prime}$ and the endpoint of $P_{1}$ produce the required long chord of the new cycle.

The case that requires explanation is when the endpoints of the matching are $s^{\prime}$ and the endpoint of $P_{1}$. Then $V\left(H^{\prime}\right)$ only yields an $\left(s^{\prime}, s^{\prime}\right)$-chord, which is not appropriate. In this case, we have to use the chord between the endpoints of $P_{1}$ instead of the edge between $s^{\prime}$ and the endpoint of $P_{1}$. It is easy to see that $V\left(H^{\prime}\right)$ together with the matching and this chord provide a long chord between $s^{\prime}$ and the other endpoint of $P_{1}$ (the one closer to $t^{\prime}$ ). Note that this endpoint is different from $s^{\prime}$, as the length of $P_{1}$ is at least two.

We have shown that if the matching size between $V\left(H^{\prime}\right)$ and $V\left(P_{1}\right)$ is at least two, then we can find a longer cycle in polynomial time. The other cases are symmetrical. Hence, $H^{\prime}$ and $H^{\prime \prime}$ satisfy the properties of (D1)-type components. This concludes the proof of the lemma. 


\section{Conclusion}

In this paper, we developed an algorithmic extension of the classical theorem of Dirac. Our main result, Theorem 3, is that LONG DIRAC CYCLE is solvable in $2^{\mathcal{O}(k+|B|)} \cdot n^{\mathcal{O}(1)}$ time on 2-connected graphs. An important step in the proof of Theorem 3 is Theorem 5. Long ERdös-Gallai $(s, t)$ PATH is solvable in $2^{\mathcal{O}(k+|B|)} \cdot n^{\mathcal{O}(1)}$ time on 2-connected graphs. In this section we provide lower bounds complementing Theorems 3 and 5 , and then conclude with open questions for further research.

\subsection{Tightness of results}

We have already observed that the dependency on $k$ in the running times of Theorems 3 and 5 is tight up to ETH. Here we show that the dependency on $|B|$ is similarly tight. Additionally, we show that for any $\varepsilon>0$, it is NP-hard to find a cycle of length at least $(1+\varepsilon) 2 \delta(G)$, meaning that our starting bound of $2 \delta(G)$ is tight. We start with the first hardness result.

Theorem 17. Unless ETH fails, there is no algorithm solving LONG DIRAC CYCLE or LONG Dirac PATH in time $2^{o(|B|)} \cdot|V(G)|^{\mathcal{O}(1)}$, even when $k=1$.

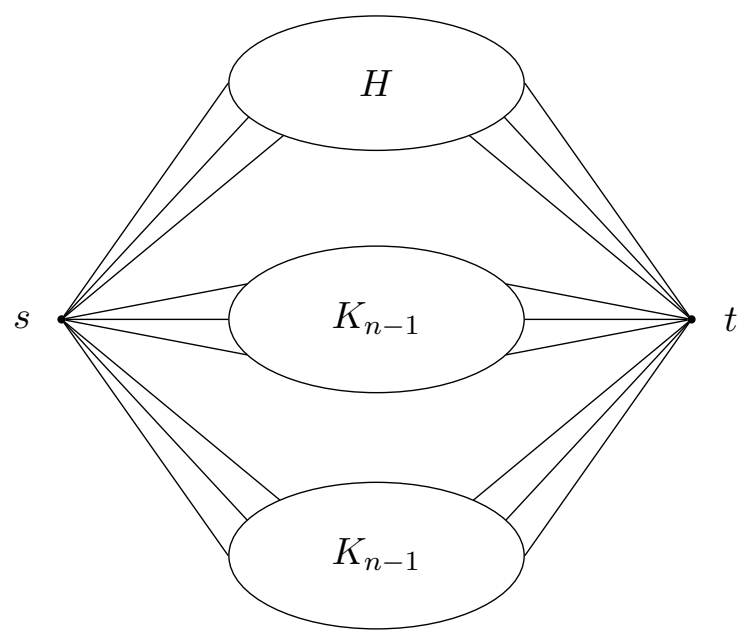

Figure 14: An illustration to the hardness reduction in Theorem 17, from Hamiltonian Path to Long Dirac Cycle. The graph $H$ is the starting Hamiltonian PATH instance. The reduction to Long DiRAC PATH looks similarly, only without the vertex $t$.

Proof. First, we show a reduction from Hamiltonian Path to Long Dirac Cycle. Consider an instance $H$ of Hamiltonian Path, let $n=|V(H)|$. Take a disjoint union of $H$ and two disjoint copies of $K_{n-1}$, the clique on $(n-1)$ vertices. Add two additional vertices $s$ and $t$ that are adjacent to all previously listed vertices (but not to each other). This finishes the description of the graph $G$ that our reduction constructs from $H$, see Figure 14 for the illustration. Finally, set $B$ to $V(H) \subset V(G)$, and $k$ to one. Observe that $2 \delta(G-B)$ and $|V(G-B)|$ are both equal to $2 n$. Our aim is now to show that $H$ has a Hamiltonian path if and only if $G$ has a cycle of length at least $2 n+1=\min \{2 \delta(G-B),|V(G)|-|B|\}+k$.

In the forward direction, if there is a Hamiltonian path $P$ in $H$, consider a Hamiltonian path $P^{\prime}$ in one of the $K_{n-1}$ components. Connect $P$ and $P^{\prime}$ in a cycle by going through the vertices $s$ and $t$. This results in a cycle of length $|V(G)|+\left|V\left(K_{n-1}\right)\right|+2=2 n+1$. 
In the other direction, let $C$ be a cycle of length at least $2 n+1$ in $G$. Since $|V(G-B)|=2 n$, $C$ necessarily intersects $B$, and since $|B|=n, C$ also intersects $V(G-B)$. Since in $G-\{s, t\}$ the set $B$ is disconnected from the rest of the graph, the cycle $C$ necessarily enters $B$ from $s$ and exits via $t$. The two $K_{n-1}$ copies are also disconnected in $G-\{s, t\}$, thus $C$ intersects exactly one of the cliques. Thus, $C$ has at most $n+1$ vertices outside of $B$. Since $|C|=2 n+1$ and $|B|=n, C$ must traverse all vertices of $B$. Since $C$ induces a path on $B$, this path is also a Hamiltonian path in $H$. This finishes the proof of correctness of the reduction.

Finally, following the reduction above, a $2^{o(|B|)} \cdot|V(G)|^{\mathcal{O}(1)}$-time algorithm for LONG DIRAC CyCle would immeidately imply a $2^{o(n)}$-time algorithm for HAMiLtonian Path since $|B|=n$ and $|V(G)|=\mathcal{O}(n)$, and the existence of the latter would contradict ETH.

For Long DiRAc PATh, the reduction follows a similar idea. From an instance $H$ of HamiltoNIAN PATH, construct a graph $G$ as follows. Take a disjoint union of $H$ and two disjoint copies of $K_{n-1}$, and add an additional apex vertex $s$. Set $B$ to be $V(H) \subset V(G)$, and set $k$ to one. Clearly, $2 \delta(G-B)=|V(G)|-|B|-1=2 n-2$. If there is a Hamiltonian path in $H$, it extends to a path of length $2 n-1$ in $G$ by continuing through $s$ into one of the cliques, as it is always possible to traverse through all vertices of the clique. On the other hand, if there is a path $P$ of length at least $2 n-1$ in $G$, it necessarily goes from $B$ to $V(G) \backslash B$, since $|B|=n$ and $|V(G) \backslash B|=2 n-1$. Such a path can only go through $s$ to one of the cliques while completely avoiding the other, since $s$ is an articulation point. Thus, outside of $B$ the path $P$ visits at most $n$ vertices, and since a path of length at least $2 n-1$ has to visit at least $2 n$ distinct vertices, $P$ necessarily traverses through all vertices of $V(H)$, yielding a Hamiltonian path in $H$. This finishes the proof for Long DiRAC РАTH.

Next, we show that the bound $2 \delta(G)$ cannot be improved unless $\mathrm{P}=\mathrm{NP}$ by proving the following theorem.

Theorem 18. For every positive $\varepsilon<1$, it is NP-complete to decide whether

(a) a 2-connected graph $G$ with two given vertices $s$ and $t$ has an $(s, t)$-path of length at least $(1+\varepsilon) \delta(G)$;

(b) a 2-connected graph $G$ has a cycle of length at least $(2+\varepsilon) \delta(G)$.

Proof. Both claims are shown by reduction from the classical Hamiltonian Path problem that is well-known to be NP-complete [GJ79]. Both reductions exploit the same idea. We first show the claim for an $(s, t)$-path and then explain how to modify the reduction for the second claim.

Let $0<\varepsilon<1$ and let $G$ be an $n$-vertex graph with $n \geq 2$. We select a positive integer $p$ such that $\lceil\varepsilon(p+1)\rceil=n$. Clearly, such an integer exists, because $\varepsilon<1$ and $n \geq 2$. Then we construct the following graph $H$ (see Figure 15).

- Construct a copy of $G$.

- Construct a vertex $t$ and make it adjacent to every vertex of $G$.

- Construct a vertex $s$.

- For every vertex $v \in V(G) \cup\{t\}$, construct a clique $Q_{v}$ with $p$ vertices and make the vertices of $Q_{v}$ adjacent to $v$ and $s$.

Notice that $H$ is 2 -connected and $\delta(H)=p+1$. We claim that $G$ is has a Hamiltonian path if and only if $H$ has an $(s, t)$-path of length at least $(1+\varepsilon) \delta(H)$. 


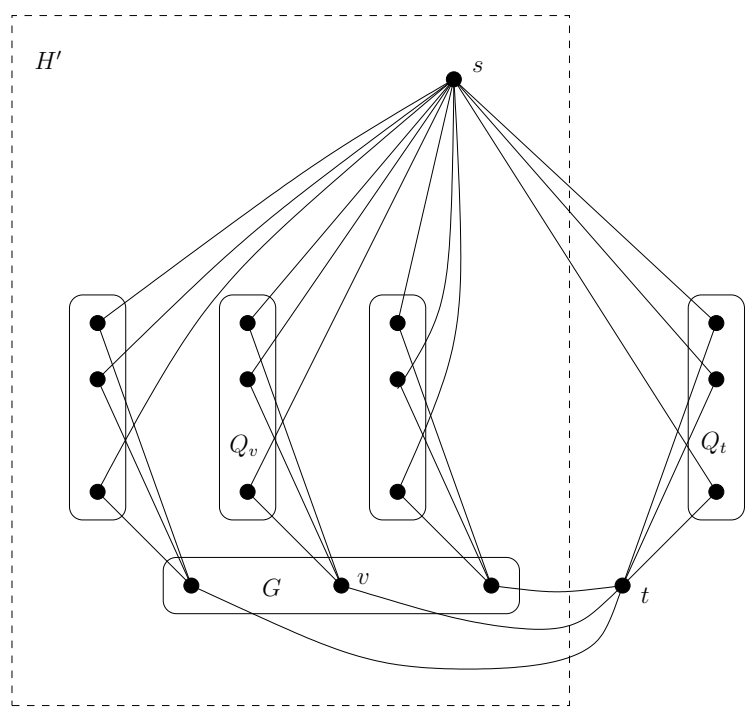

Figure 15: Construction of $H$ and $H^{\prime}$.

In one direction, let $P$ be a Hamiltonian path in $G$ and denote by $x$ and $y$ its endpoints. Because $Q_{x}$ is a clique, $H$ has an $(s, x)$-path $R$ with $V(R)=Q_{x} \cup\{s, x\}$. That is, $R$ is a Hamiltonian path in $H\left[Q_{x} \cup\{s, x\}\right]$. Consider path $P^{\prime}$ obtained by concatenating $R, P$, and $y t$. Then $P^{\prime}$ is an $(s, t)$-path in $H$. Observe that the length of $P^{\prime}$ is

$$
(p+1)+(n-1)+1=p+n+1=p+1+\lceil\varepsilon(p+1)\rceil \geq(1+\varepsilon)(p+1)=(1+\varepsilon) \delta(H)
$$

as required.

For the opposite direction, assume that $P^{\prime}$ is an $(s, t)$-path in $H$ of length at least $(1+\varepsilon) \delta(H)$. Then the length of $P^{\prime}$ is at least

$$
\lceil(1+\varepsilon) \delta(H)\rceil=\delta(H)+\lceil\varepsilon \delta(G)\rceil=(p+1)+\lceil\varepsilon(p+1)\rceil=p+1+n .
$$

By the construction of $H, P^{\prime}$ is the concatenation of paths $R$ and $S$ such that $R$ is an $(s, v)$-path for some $v \in V(G) \cup\{t\}$ where $V(R) \subseteq V\left(Q_{v}\right) \cup\{s, v\}$ and $S$ is a $(v, t)$-path with $V(S) \subseteq V(G) \cup\{t\}$. The length of $R$ is at most $p+1=\delta(H)$. Therefore, the length of $S$ is at least $n$. Consider the path $P$ obtained from $S$ by deleting $t$. We have that $V(P) \subseteq V(G)$ and the length of $P$ is at least $n-1$. We obtain that $P$ is a Hamiltonian path in $G$. This concludes the proof of the first claim.

The proof of (b) is similar. Let $0<\varepsilon<1$ and let $G$ be an $n$-vertex connected graph with $n \geq 3$. Now we select a positive integer $p$ such that $\lceil\varepsilon(p+1)\rceil=n-1$. We construct graph $H^{\prime}$ that is, in fact, the graph obtained from $H$ constructed above by deleting $t$ and the vertices of $Q_{t}$ (see Figure 15). Formally, $H^{\prime}$ is constructed as follows.

- Construct a copy of $G$.

- Construct a vertex $s$.

- For every vertex $v \in V(G)$, construct a clique $Q_{v}$ with $p$ vertices and make the vertices of $Q_{v}$ adjacent to $v$ and $s$.

Because $G$ is a connected graph with at least three vertices, $\delta\left(H^{\prime}\right)=p+1$. Because $G$ is connected, $H^{\prime}$ is 2-connected. We claim that $G$ has a Hamiltonian path if and only if $H^{\prime}$ has a cycle of length at least $(2+\varepsilon) \delta(H)$. 
Suppose that $P$ is a Hamiltonian path in $G$ and let $x$ and $y$ be its endpoints. Note that since $G$ has at least two vertices, $x \neq y$. Because $Q_{x}$ and $Q_{y}$ are cliques, $H$ has an $(s, x)$-path $R_{x}$ with $V\left(R_{x}\right)=Q_{x} \cup\{s, x\}$ and a $(y, s)$-path $R_{y}$ with $V\left(R_{y}\right)=Q_{y} \cup\{s, y\}$. Observe that the concatenation of $R_{x}, P$, and $R_{y}$ is a cycle. Denote this cycle by $C$. The length of $C$ is

$$
(p+1)+(n-1)+(p+1)=2(p+1)+n-1=2(p+1)+\lceil\varepsilon(p+1)\rceil \geq(2+\varepsilon)(p+1)=(1+\varepsilon) \delta(H) .
$$

Finally, let $C$ be a cycle of $G$ of length at least $(2+\varepsilon) \delta(H)$. Then the length of $C$ is at least

$$
\lceil(2+\varepsilon) \delta(H)\rceil=2 \delta(H)+\lceil\varepsilon \delta(G)\rceil=2(p+1)+\lceil\varepsilon(p+1)\rceil=2(p+1)+n-1 .
$$

Suppose that $s \notin V(C)$. Then, by the construction of $H^{\prime}$, either $C$ is a cycle in $H^{\prime}\left[Q_{v} \cup\{v\}\right]$ for some $v \in V(G)$ or $C$ is a cycle of $G$. In the first case the length of $C$ is at most $\left|Q_{v}\right|+1=p+1$, and in the second case the length of $C$ is at most $n$. In both cases, we have that the length of $C$ is strictly less that $2(p+1)+n-1$. This implies that $s \in V(C)$. If $|V(C) \cap V(G)| \leq 1$, then $V(C) \subseteq Q_{v} \cup\{s, v\}$ for some $v \in V(G)$. However, $|V(C)| \leq p+2<2(p+1)+n-1$ in this case. Hence, $|V(C) \cap V(G)| \geq 2$. Then the construction of $H^{\prime}$ implies that $|V(C) \cap V(G)|=2$. Let $\{x, y\}=V(C) \cap V(G)$. It is easy to verify that $C$ can be seen as the concatenation of three paths $R_{x}, P$, and $R_{y}$, where $R_{x}$ is an $(s, x)$-path with $V\left(R_{x}\right) \subseteq Q_{x} \cup\{s, x\}, P$ is an $(x, y)$-path in $G$, and $R_{y}$ is a $(y, s)$-path with $V\left(R_{y}\right) \subseteq Q_{y} \cup\{s, y\}$. The length of $R_{x}$ and the length of $R_{y}$ is at most $p+1$. This means, that the length of $P$ is at least $n-1$. Therefore, $P$ is a Hamiltonian path in $G$. This concludes the proof.

For simplicity, we proved Theorem 18 for the case when $\varepsilon<1$ but let us remark that the claim also holds for $\varepsilon \geq 1$. Moreover, it can be assumed that $\varepsilon$ not a constant but an appropriate function of $\delta(G)$ like $\varepsilon(\delta)=\delta^{c}$ for some constant $c>-1$.

\subsection{Open questions}

Dirac's theorem is the first fundamental result in Extremal Hamiltonian Graph Theory. The area contains many deep and interesting theorems but it remains largely unexplored from the algorithmic perspective. Here we present several open questions hoping that these questions would trigger further research in this fascinating area.

The first question is from $\left[\mathrm{FGL}^{+} 20 \mathrm{a}\right]$. Recall that the average degree of a graph $G$ is

$$
\frac{1}{|V(G)|} \sum_{v \in V(G)} \operatorname{deg}_{G}(v)=2|E(G)| /|V(G)| .
$$

The following was shown by Erdős and Gallai [EG59].

Proposition 4 ([EG59]). Every graph $G$ with average degree at least $d \geq 2$ has a cycle of length at least $d$.

Similarly, it can be shown that a graph $G$ with average degree at least $d$ has a path of length at least $d$. This leads to the following question.

Open Question 1 (Path/cycle above average degree). Given a 2-connected (connected, respectively) graph $G$ and a nonnegative integer $k$, how difficult is to decide whether $G$ has a cycle (a path, respectively) of length at least $2|E(G)| /|V(G)|+k$ ? 
We do not know whether the problem is FPT parameterized by $k, \mathrm{~W}$ [1]-hard, or Para-NP. Even the simplest variant of the question: whether a path of length $2|E(G)| /|V(G)|+1$ could be computed in polynomial time, is open.

Our second open question concerns the problem of finding a cycle containing a specified set of vertices. The study of this problem can be traced back to another fundamental theorem of Dirac from 1960s about the existence of a cycle in $h$-connected graph passing through a given set of $h$ vertices [Dir60]. According to Kawarabayashi [Kaw08] “...cycles through a vertex set or an edge set are one of central topics in all of graph theory." Such type of problems have been a popular and important topic in algorithms as well. See, e.g., Björklund, Husfeldt and Taslaman [BHT12] and Wahlström [Wah13, and Kawarabayashi Kaw08.

In Extremal Hamiltonian Graph Theory, the following theorem of Egawa, Glas, and Locke [EGL91. is well-known.

Theorem 19 ([EGL91]). Let $G$ be an $h$-connected graph, $h \geq 2$, with minimum degree $d$, and at least $2 d-1$ vertices. Let $X$ be a set of $h$ vertices of $G$. Then $G$ has a cycle $C$ of length at least $2 d$ such that every vertex of $X$ is on $C$.

This brings us to the following algorithmic problem.

Open Question 2 (Cycle above Egawa, Glas, and Locke condition). Given an $h$-connected graph $G$, a set of vertices $X \subseteq V(G)$ of size $h$, and a nonnegative integer $k$, how difficult is to decide whether $G$ has a cycle of length at least $2 \delta(G)+k$ containing every vertex of $X$ ?

As for Open Question 1 the question is open even for $k=1$.

Finally, let us mention the area of directed graphs; we refer to the book of Bang-Jensen and Gutin [BG09] and the survey of Bermond and Thomassen [BT81] for extremal theorems for directed graphs. In particular, the classical result of Ghouila-Houri [GH60] from 1960, generalizes Theorem 1. Recall that a digraph $D$ is strong if for every two vertices $u$ and $v, D$ has directed $(u, v)$ and $(v, u)$-path, and the degree $\operatorname{deg}_{D}(v)$ of a vertex $v$ is the sum of its in-degree $\operatorname{deg}_{D}^{-}(v)$ and out-degree $\operatorname{deg}_{D}^{+}(v)$.

Theorem 20 ([GH60]). If for every vertex $v$ of a strong digraph $D$ with $n$ vertices $\operatorname{deg}_{D}(v) \geq n$, then $D$ has a Hamiltonian cycle.

The following question is the variant of the question discussed by Jansen, Kozma and Nederlof in JKN19 for undirected graphs.

Open Question 3 (Cycle above Ghouila-Houri condition). Given an $n$-vertex strong digraph $D$ and a nonnegative integer $k$ such that at least $n-k$ vertices have degree at least $n$, how difficult is to decide whether $D$ is Hamiltonian?

Again, the simplest variant-whether there is a polynomial time algorithm for for $k=1$-is open. We also do not know the complexity of the problem when every vertex has degree at least $n-k$.

A digraph $D$ with at least two vertices is 2-connected if it is strong and remains strong after deleting an arbitrary vertex. Thomassen in [Tho81] proved the following analog of Theorem 2 .

Theorem 21 ([Tho81]). Let $D$ be a 2-connected digraph with at least $2 d+1$ vertices such that $\operatorname{deg}_{D}^{-}(v) \geq d$ and $\operatorname{deg}_{D}^{+}(v) \geq d$ for every $v \in V(D)$. Then $D$ contains a cycle of length at least $2 d$.

Whether Thomassen's theorem can be extended algorithmically is our last open question. 
Open Question 4 (Cycle above Thomassen condition). What is the (parameterized) complexity of the following problem. Given a 2-connected digraph $D$ such that $\operatorname{deg}_{D}^{-}(v) \geq d$ and $\operatorname{deg}_{D}^{+}(v) \geq d$ for every $v \in V(D)$, and a nonnegative integer $k$. Decide whether $D$ contains a cycle of length at least $2 d+k$.

As in Questions 1 3, even the existence of a polynomial time algorithm for $k=1$ in Question 4 is open.

\section{References}

$\left[\mathrm{AGK}^{+} 10\right]$ Noga Alon, Gregory Gutin, Eun Jung Kim, Stefan Szeider, and Anders Yeo. Solving MAX-r-SAT above a tight lower bound. In Proceedings of the 21st Annual ACM-SIAM Symposium on Discrete Algorithms (SODA), pages 511-517. SIAM, 2010.

[AYZ95] Noga Alon, Raphael Yuster, and Uri Zwick. Color-coding. J. ACM, 42(4):844-856, 1995.

[BB93] Béla Bollobás and Graham Brightwell. Cycles through specified vertices. Combinatorica, 13(2):147-155, 1993.

[BC76] J. A. Bondy and V. Chvátal. A method in graph theory. Discrete Math., 15(2):111-135, 1976.

[BCDF19] Ivona Bezáková, Radu Curticapean, Holger Dell, and Fedor V. Fomin. Finding detours is fixed-parameter tractable. SIAM J. Discrete Math., 33(4):2326-2345, 2019.

[BG09] Jørgen Bang-Jensen and Gregory Z. Gutin. Digraphs - Theory, Algorithms and Applications, Second Edition. Springer Monographs in Mathematics. Springer, 2009.

[BHKK10] Andreas Björklund, Thore Husfeldt, Petteri Kaski, and Mikko Koivisto. Narrow sieves for parameterized paths and packings. CoRR, abs/1007.1161, 2010.

[BHT12] Andreas Björklund, Thore Husfeldt, and Nina Taslaman. Shortest cycle through specified elements. In Proceedings of the 22nd Annual ACM-SIAM Symposium on Discrete Algorithms (SODA), pages 1747-1753. SIAM, 2012.

[Bjö14] Andreas Björklund. Determinant sums for undirected hamiltonicity. SIAM J. Comput., 43(1):280-299, 2014.

[Bol78] Béla Bollobás. Extremal graph theory, volume 11 of London Mathematical Society Monographs. Academic Press, Inc. [Harcourt Brace Jovanovich, Publishers], LondonNew York, 1978.

[Bol95] Béla Bollobás. Extremal graph theory. In Handbook of combinatorics, Vol. 1, 2, pages 1231-1292. Elsevier Sci. B. V., Amsterdam, 1995.

[Bon95] J. A. Bondy. Basic graph theory: paths and circuits. In Handbook of combinatorics, Vol. 1, 2, pages 3-110. Elsevier Sci. B. V., Amsterdam, 1995.

[BT81] Jean-Claude Bermond and Carsten Thomassen. Cycles in digraphs- a survey. J. Graph Theory, 5(1):1-43, 1981. 
[CCC06] Leizhen Cai, Siu Man Chan, and Siu On Chan. Random separation: A new method for solving fixed-cardinality optimization problems. In Proceedings of the 2nd International Workshop on Parameterized and Exact Computation (IWPEC), volume 4169 of Lecture Notes in Comput. Sci., pages 239-250. Springer, 2006.

[CFK $\left.{ }^{+} 15 \mathrm{a}\right]$ Marek Cygan, Fedor V. Fomin, Łukasz Kowalik, Daniel Lokshtanov, Dániel Marx, Marcin Pilipczuk, Michał Pilipczuk, and Saket Saurabh. Parameterized Algorithms. Springer, 2015.

$\left[\mathrm{CFK}^{+}\right.$15b] Marek Cygan, Fedor V. Fomin, Lukasz Kowalik, Daniel Lokshtanov, Dániel Marx, Marcin Pilipczuk, Michal Pilipczuk, and Saket Saurabh. Parameterized Algorithms. Springer, 2015.

$\left[\mathrm{CJM}^{+} 13\right]$ Robert Crowston, Mark Jones, Gabriele Muciaccia, Geevarghese Philip, Ashutosh Rai, and Saket Saurabh. Polynomial kernels for lambda-extendible properties parameterized above the Poljak-Turzik bound. In IARCS Annual Conference on Foundations of Software Technology and Theoretical Computer Science (FSTTCS), volume 24 of Leibniz International Proceedings in Informatics (LIPIcs), pages 43-54, Dagstuhl, Germany, 2013. Schloss Dagstuhl-Leibniz-Zentrum fuer Informatik.

[Die17] Reinhard Diestel. Graph theory, volume 173 of Graduate Texts in Mathematics. Springer-Verlag, Berlin, 5th edition, 2017.

[Dir52] G. A. Dirac. Some theorems on abstract graphs. Proc. London Math. Soc. (3), 2:69-81, 1952.

[Dir60] Gabriel Andrew Dirac. In abstrakten Graphen vorhandene vollständige 4-Graphen und ihre Unterteilungen. Math. Nachr., 22:61-85, 1960.

[EG59] P. Erdős and T. Gallai. On maximal paths and circuits of graphs. Acta Math. Acad. Sci. Hungar, 10:337-356, 1959.

[EGL91] Y Egawa, R Glas, and S.C Locke. Cycles and paths through specified vertices in k-connected graphs. Journal of Combinatorial Theory, Series B, 52(1):20-29, May 1991.

$\left[\mathrm{FGL}^{+}\right.$20a] Fedor V. Fomin, Petr A. Golovach, Daniel Lokshtanov, Fahad Panolan, Saket Saurabh, and Meirav Zehavi. Going far from degeneracy. SIAM J. Discrete Math., 34(3):15871601, 2020.

$\left[\mathrm{FGL}^{+}\right.$20b] Fedor V. Fomin, Petr A. Golovach, Daniel Lokshtanov, Fahad Panolan, Saket Saurabh, and Meirav Zehavi. Parameterization Above a Multiplicative Guarantee. In Proceedings of the 11th Innovations in Theoretical Computer Science Conference (ITCS), volume 151 of Leibniz International Proceedings in Informatics (LIPIcs), pages 39:1-39:13. Schloss Dagstuhl-Leibniz-Zentrum fuer Informatik, 2020.

[FK13] Fedor V. Fomin and Petteri Kaski. Exact exponential algorithms. Commun. ACM, $56(3): 80-88,2013$.

$\left[\mathrm{FLP}^{+} 18\right] \quad$ Fedor V. Fomin, Daniel Lokshtanov, Fahad Panolan, Saket Saurabh, and Meirav Zehavi. Long directed $(s, t)$-path: FPT algorithm. Inf. Process. Lett., 140:8-12, 2018. 
[FLPS16] Fedor V. Fomin, Daniel Lokshtanov, Fahad Panolan, and Saket Saurabh. Efficient computation of representative families with applications in parameterized and exact algorithms. J. ACM, 63(4):29:1-29:60, 2016.

[GH60] Alain Ghouila-Houri. Une condition suffisante d'existence d'un circuit hamiltonien. $C$. R. Acad. Sci. Paris, 251:495-497, 1960.

[GJ79] M. R. Garey and David S. Johnson. Computers and Intractability: A Guide to the Theory of NP-Completeness. W. H. Freeman, 1979.

[GKLM11] Gregory Gutin, Eun Jung Kim, Michael Lampis, and Valia Mitsou. Vertex cover problem parameterized above and below tight bounds. Theory of Computing Systems, 48(2):402-410, 2011.

[Gör00] Frank Göring. Short proof of Menger's theorem. Discrete Mathematics, 219(1-3):295296, 2000.

[GP16a] Shivam Garg and Geevarghese Philip. Raising the bar for vertex cover: Fixedparameter tractability above a higher guarantee. In Proceedings of the Twenty-Seventh Annual ACM-SIAM Symposium on Discrete Algorithms (SODA), pages 1152-1166. SIAM, 2016.

[GP16b] Gregory Z. Gutin and Viresh Patel. Parameterized traveling salesman problem: Beating the average. SIAM J. Discrete Math., 30(1):220-238, 2016.

[GRSY07] Gregory Z. Gutin, Arash Rafiey, Stefan Szeider, and Anders Yeo. The linear arrangement problem parameterized above guaranteed value. Theory Comput. Syst., 41(3):521-538, 2007.

[GvIMY12] Gregory Gutin, Leo van Iersel, Matthias Mnich, and Anders Yeo. Every ternary permutation constraint satisfaction problem parameterized above average has a kernel with a quadratic number of variables. J. Computer and System Sciences, 78(1):151-163, 2012.

[H9̈2] Roland Häggkvist. On the structure of non-Hamiltonian graphs. I. Combin. Probab. Comput., 1(1):27-34, 1992.

[IPZ01] Russell Impagliazzo, Ramamohan Paturi, and Francis Zane. Which problems have strongly exponential complexity. J. Computer and System Sciences, 63(4):512-530, 2001.

[JKN19] Bart M. P. Jansen, László Kozma, and Jesper Nederlof. Hamiltonicity below Dirac's condition. In Proceedings of the 45th International Workshop on Graph-Theoretic Concepts in Computer Science (WG), volume 11789 of Lecture Notes in Computer Science, pages 27-39. Springer, 2019.

[Kaw08] Ken-ichi Kawarabayashi. An improved algorithm for finding cycles through elements. In Proceedings of the 13th International Conference on Integer Programming and Combinatorial Optimization (IPCO), volume 5035 of Lecture Notes in Comput. Sci., pages 374-384. Springer, 2008. 
[Kou08] Ioannis Koutis. Faster algebraic algorithms for path and packing problems. In Proceedings of the 35th International Colloquium on Automata, Languages and Programming (ICALP), volume 5125 of Lecture Notes in Comput. Sci., pages 575-586. Springer, 2008.

[KW16] Ioannis Koutis and Ryan Williams. Algebraic fingerprints for faster algorithms. Commun. ACM, 59(1):98-105, 2016.

[Li13] Hao Li. Generalizations of Dirac's theorem in Hamiltonian graph theory - a survey. Discrete Math., 313(19):2034-2053, 2013.

[LN21] Binlong Li and Bo Ning. A strengthening of Erdős-Gallai Theorem and proof of Woodall's conjecture. J. Combin. Theory Ser. B, 146:76-95, 2021.

$\left[\mathrm{LNR}^{+}\right.$14] Daniel Lokshtanov, N. S. Narayanaswamy, Venkatesh Raman, M. S. Ramanujan, and Saket Saurabh. Faster parameterized algorithms using linear programming. ACM Trans. Algorithms, 11(2):15:1-15:31, 2014.

[Loc83] Stephen Charles Locke. Extremal Properties Of Paths, Cycles And K-Colourable Subgraphs Of Graphs. PhD thesis, University of Waterloo, 1983.

[Loc85] Stephen C Locke. A generalization of Dirac's theorem. Combinatorica, 5(2):149-159, 1985.

[Men27] Karl Menger. Zur allgemeinen kurventheorie. Fundamenta Mathematicae, 10(1):96$115,1927$.

[Mey73] M. Meyniel. Une condition suffisante d'existence d'un circuit Hamiltonien dans un graphe oriente. J. Combinatorial Theory Ser. B, 14:137-147, 1973.

[MR99] Meena Mahajan and Venkatesh Raman. Parameterizing above guaranteed values: Maxsat and maxcut. J. Algorithms, 31(2):335-354, 1999.

[MRS09] Meena Mahajan, Venkatesh Raman, and Somnath Sikdar. Parameterizing above or below guaranteed values. J. Computer and System Sciences, 75(2):137-153, 2009.

[NSS95] Moni Naor, Leonard J. Schulman, and Aravind Srinivasan. Splitters and near-optimal derandomization. In Proceedings of the 36th Annual Symposium on Foundations of Computer Science (FOCS 1995), pages 182-191. IEEE, 1995.

[NW71] C. St. J. A. Nash-Williams. Edge-disjoint Hamiltonian circuits in graphs with vertices of large valency. In Studies in Pure Mathematics (Presented to Richard Rado), pages 157-183. Academic Press, London, 1971.

[Ore60] Oystein Ore. Note on Hamilton circuits. Amer. Math. Monthly, 67:55, 1960.

[P6́2] L. Pósa. A theorem concerning Hamilton lines. Magyar Tud. Akad. Mat. Kutató Int. Közl., 7:225-226, 1962.

[Tho81] Carsten Thomassen. Long cycles in digraphs. Proc. London Math. Soc. (3), 42(2):231251, 1981.

[Tsu19] Dekel Tsur. Faster deterministic parameterized algorithm for $k$-path. Theor. Comput. Sci., 790:96-104, 2019. 
[Wah13] Magnus Wahlström. Abusing the tutte $\mathrm{m}=$ matrix: An algebraic instance compression for the $k$-set-cycle problem. In Proceedings of the 30th International Symposium on Theoretical Aspects of Computer Science (STACS), volume 20 of Leibniz International Proceedings in Informatics (LIPIcs), pages 341-352. Schloss Dagstuhl - LeibnizZentrum fuer Informatik, 2013.

[Wil09] Ryan Williams. Finding paths of length $k$ in $O^{*}\left(2^{k}\right)$ time. Inf. Process. Lett., 109(6):315-318, 2009.

[Wil19] David Williamson. Network Flow Algorithms. Cambridge University Press, 2019.

[Zeh16] Meirav Zehavi. A randomized algorithm for long directed cycle. Inf. Process. Lett., 116(6):419-422, 2016. 